\title{
Lessons learned in the selection and development of test cases for the Aeroelastic Prediction Workshop: Rectangular Supercritical Wing
}

\author{
Jennifer Heeg, ${ }^{*}$ Pawel Chwalowski, ${ }^{\dagger}$ Carol D. Wieseman ${ }^{\ddagger}$ \\ Jennifer P. Florance ${ }^{\S}$ and David M. Schuster ${ }^{\mathbb{I}}$ \\ NASA Langley Research Center, Hampton, VA 23681-2199
}

\begin{abstract}
The Aeroelastic Prediction Workshop brought together an international community of computational fluid dynamicists as a step in defining the state of the art in computational aeroelasticity. The Rectangular Supercritical Wing (RSW) was chosen as the first configuration to study due to its geometric simplicity, perceived simple flow field at transonic conditions and availability of an experimental data set containing forced oscillation response data. Six teams performed analyses of the RSW; they used Reynolds-Averaged Navier-Stokes flow solvers exercised assuming that the wing had a rigid structure. Both steady-state and forced oscillation computations were performed by each team. The results of these calculations were compared with each other and with the experimental data. The steady-state results from the computations capture many of the flow features of a classical supercritical airfoil pressure distribution. The most dominant feature of the oscillatory results is the upper surface shock dynamics. Substantial variations were observed among the computational solutions as well as differences relative to the experimental data. Contributing issues to these differences include substantial wind tunnel wall effects and diverse choices in the analysis parameters.
\end{abstract}

\section{Nomenclature}

$\begin{array}{ll}C_{p} & \text { Coefficient of pressure } \\ M & \text { Mach number }\end{array}$

Symbols

$\alpha \quad$ Angle of attack

$\delta \quad$ Boundary layer thickness

$\delta^{*} \quad$ Displacement thickness

$\theta \quad$ Momentum thickness

$\omega, \mathrm{f} \quad$ Frequency - radians/second, $\mathrm{Hz}$

AePW Aeroelastic Prediction Workshop

BSCW Benchmark Supercritical Wing

CAE Computational Aeroelasticity

CFD Computational Fluid Dynamics

DFT Discrete Fourier Transform

DPW Drag Prediction Workshop

FRF Frequency Response Function

HIRENASD HIgh REynolds Number AeroStructural Dynamics

HiLiftPW High Lift Prediction Workshop

OC AePW Organizing Committee

\footnotetext{
*Senior Research Engineer, Aeroelasticity Branch, MS 340, Senior Member AIAA

${ }^{\dagger}$ Senior Research Engineer, Aeroelasticity Branch, MS 340, Senior Member AIAA

\#Senior Research Engineer, Aeroelasticity Branch, MS 340, Associate Fellow AIAA

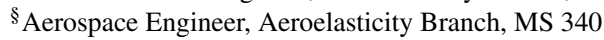

"NASA Technical Fellow for Aerosciences, NASA Engineering and Safety Center, Associate Fellow, AIAA
} 


\section{Introduction}

$\mathrm{T}$ he credibility of computational methods has improved in recent years due to independent, individual verification and validation efforts, but multi-analyst code-to-code comparisons that can be used to assess the overall current state-of-the-art in computational aeroelasticity (CAE) are limited. The idea for an Aeroelastic Prediction Workshop (AePW) series was conceived in 2009, based on the success of two other workshop series that have been conducted over the past decade: the Drag Prediction Workshop ${ }^{1,2}$ (DPW) series and the High Lift Prediction Workshop $^{3,4}$ (HiLiftPW) series. The genesis of the AePW and an informational overview of the workshop is provided in reference Heeg et al., ${ }^{5}$ and a summary of the comparison data sets is presented in reference Schuster et al. ${ }^{6}$ The intent for the AePW is to provide a forum for code-to-code comparisons involving predictions of nonlinear aeroelastic phenomena and to stimulate upgrades for existing codes and the development of new codes.

For code validations in general, the type of aerodynamic and/or aeroelastic phenomena selected for analysis is important since that validation process typically progresses from simpler to more challenging cases. For the AePW series, the approach being taken is to utilize existing experimental data sets in a building-block approach to incrementally validate targeted aspects of CAE tools. Each "block" will represent a component of a more complex nonlinear unsteady aeroelastic problem, isolating it such that the contributing physics can be thoroughly investigated. The challenge selected for the first AePW was the accurate prediction of unsteady aerodynamic phenomena and their effect on the aeroelastic response of essentially "rigid," geometrically simple models, with an additional foray into systems with weak coupling between the fluid and the structure. Results from this first workshop will help guide the direction of future workshops, with analyses extending to include predictions of static aeroelastic properties, limit-cycle oscillation (LCO), flutter, buffet, and control surface effectiveness with increasingly complicated flow fields and model geometries.

The AIAA Aeroelastic Prediction Workshop (AePW) was held in conjunction with the 53rd AIAA Structures, Structural Dynamics, and Materials Conference on April 21-22, 2012, in Honolulu, Hawaii. The computational community was challenged to analyze three configurations and present their results at the workshop. The first two configurations involved "rigid" geometries in static and/or forced motion boundary conditions with attached, fully separated, and transiently separated flows at transonic conditions. The first configuration was the NASA Rectangular Supercritical Wing (RSW), which was tested in the NASA Langley Transonic Dynamics Tunnel ${ }^{7,8}$ (TDT) in 1982. For this experiment, a simple, rectangular, "rigid" supercritical wing was sidewall-mounted to a small splitter plate and oscillated in pitch, exhibiting a moderate shock and boundary-layer interaction. The second configuration utilized was the NASA Benchmark Supercritical Wing (BSCW), which was tested in the NASA Langley TDT in 2000. This data set was acquired for a "rigid" rectangular planform similar to the RSW that was sidewall-mounted to a large splitter plate assembly and oscillated in pitch via the TDT Oscillating Turntable (OTT), ${ }^{9}$ exhibiting a strong shock and boundary-layer-induced separated flow at a moderate angle of attack. The third configuration considered for AePW extended the unsteady aerodynamic prediction to a weakly-coupled aeroelastic test case involving a more complex geometry: the HIgh REynolds Number AeroStructural Dynamics (HIRENASD) Project configuration. The HIRENASD model was tested in the European Transonic Windtunnel (ETW) in 2006. For this experiment, a "rigid," semi-span, transport-type wing configuration was mounted to the tunnel ceiling and oscillated at or near the frequency of the first bending mode, the second bending mode, or the first torsion mode.

The main focus of this paper is an in-depth exploration of the RSW effort for AePW. A description of the RSW configuration and the rationale for its selection as a test case will be presented first. Computational fluid dynamics (CFD) model development and analysis methodology will then be discussed, with an emphasis on lessons learned. The resulting analytical predictions and corresponding experimental data will then be presented and examined. The full set of comparison data are shown in the appendices to this paper. Appendices A and B contain the unforced system data at $\alpha=2^{\circ}$ and $4^{\circ}$, respectively. Appendices $C$ and D contain the forced oscillation data at 10 and $20 \mathrm{~Hz}$, respectively. Appendix E contains mean pressure distributions generated by examining time-accurate forced oscillation results. 


\section{Background and preparation}

\section{II.A. Rectangular Supercritical Wing (RSW)}

The initial configuration utilized in the AePW was the NASA Rectangular Supercritical Wing (RSW). ${ }^{10-13}$ The RSW model, shown in figure 1, has a simple, unswept, rectangular, 24- $\mathrm{x} 48$-inch planform with a wing tip of revolution and a constant 12-percent-thick supercritical airfoil section. A photograph of the model installed in the TDT is shown in figure 2a. The model was mounted on the TDT east wall to a relatively small, 48- $\mathrm{x} 98$-inch splitter plate, as shown in figures 1 and 2a, which offset the model 6 inches from the wall. The model was designed to be structurally rigid, with a first bending mode frequency of $34.8 \mathrm{~Hz}$. Model instrumentation included 4 chordwise rows of unsteady pressures at span stations $0.309,0.588,0.809$, and 0.951 (with 29 pressures per chord), 8 accelerometers, and 1 potentiometer, as shown in figure $2 b$.

For the RSW test in the TDT, data was acquired in an R-12 test medium at Mach numbers ranging from 0.40 to 0.90 , dynamic pressures (q) of 175 to $215 \mathrm{psf}$, and angles of attack ( $\alpha$ ) from -1 to $7^{\circ}$. Steady data was obtained with the model held at a fixed angle of attack. Dynamic data was acquired via forced oscillation of the model in pitch about the 46-percent chord location with a shaft-driven rotary hydraulic actuator. Pitch frequencies of 5, 10, 15, and $20 \mathrm{~Hz}$ were tested at amplitudes ranging from $0.50^{\circ}$ to $1.50^{\circ}$. For most of the wind-tunnel test, flow transition was free; however, a few points were measured with a transition strip of size 60 grit affixed at 6-percent chord on both the upper and lower surfaces.

For AePW, four test cases were selected from the RSW data set. As shown in table 1, the test cases consisted of two steady data sets and two dynamic data sets. All cases were obtained at Mach 0.825 and a chord Reynolds number of 4.0 million, with the transition strips in place. The two static cases were chosen to focus on the steady solutions and their variations. Test case number one had a $2^{\circ}$ angle of attack and was anticipated to produce a moderate-strength shock, with the additional potential of shock-separated flow. The second static test case at $4^{\circ}$ angle of attack was anticipated to produce a strong shock, with even greater potential for shock-separated flow. The two dynamic, forcedoscillation test cases were chosen to examine unsteady aerodynamic predictions and their variations. Both dynamic cases involved forced pitch oscillations of $1^{\circ}$ about a $2^{\circ}$ mean angle of attack, which corresponded to the first steady test case and introduced a wing-loading bias for which code-to-code comparisons could be made. The difference between test cases three and four was in their frequency of oscillation (10 and $20 \mathrm{~Hz}$, respectively), allowing for the evaluation of frequency effects.

The RSW configuration was originally chosen for its geometric simplicity and its low-to-moderately challenging transonic aerodynamic characteristics. It was anticipated to provide "slam dunk" test cases for AePW. That's not to say that RSW was an ideal data set, though. There were five known deficiencies when this configuration was selected for AePW. First, due to the age of the data set, not all of the pertinent test information was available and/or accessible. This included time history data records and the model itself, which no longer exists. The engineers who conducted the test were also unavailable for consultation. Second, the splitter plate was very small, only approximately 4 chords by 2 chords in size. Third, the wind-tunnel wall slots were open during RSW testing, as can be seen in figure 2. Since this test was completed, open slots have been demonstrated to have an effect on steady lift curve slope when testing large models mounted directly on the wind tunnel wall. It is unclear how significant the influence of the open slots might have been on the RSW data set. Fourth, the spacing between pressures transducers is larger than desirable for examining shock motion. This is a problem common to most models that have been tested up to the current time. Without prior knowledge of the shock location or with the requirement to test at multiple Mach numbers which will change the shock location, this issue is difficult to solve. Finally, there were several pressure sensors of questionabe health that will be discussed in more detail later in this paper. None of these deficiencies were originally anticipated to have an effect on the workshop analyses.

However, an unforeseen influence of the wind-tunnel wall did ultimately make this case significantly more difficult than anticipated. Fifteen years after the RSW test, a calibration of the TDT was conducted, following a heavy-gas conversion from R-12 to R-134a. During this calibration, a boundary-layer rake mounted on the east wall measured a boundary-layer thickness of 12 inches at the test conditions associated with the RSW test cases. ${ }^{14}$ As previously mentioned, the RSW was mounted to the east wall, with only a 6-inch offset afforded by the splitter plate, well within this measured wall boundary layer. Preliminary AePW analyses of the RSW showed that the inboard pressure distributions were highly affected by the presence of this wind-tunnel wall boundary layer, and as a result, the RSW test cases were considerably more challenging than intended. The impact of this on the analysis efforts will be addressed in more detail later in this paper. 


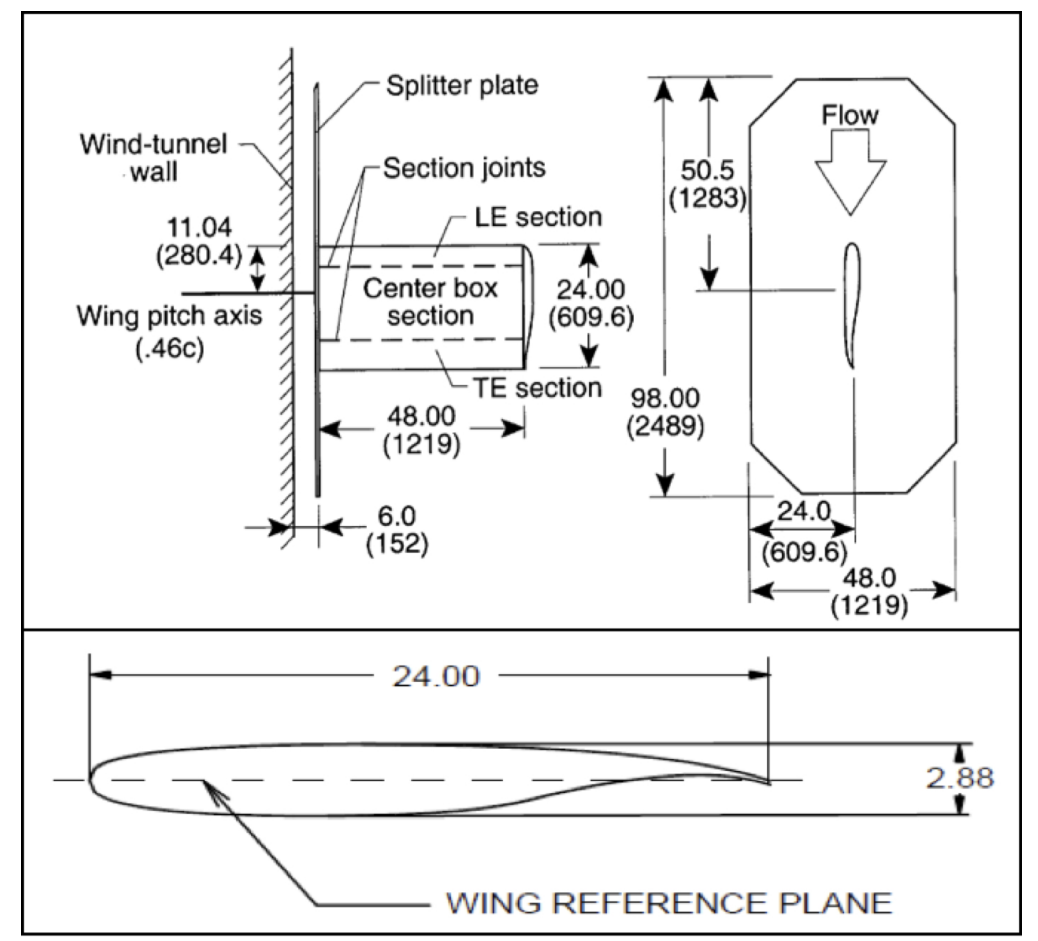

Figure 1. RSW configuration schematic.

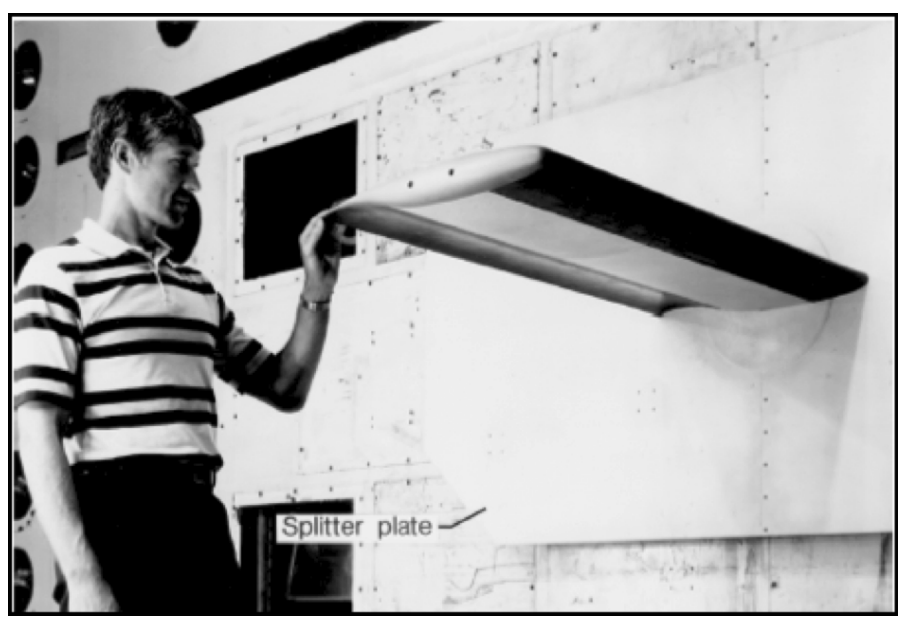

(a) Photograph of the RSW model mounted in the TDT.

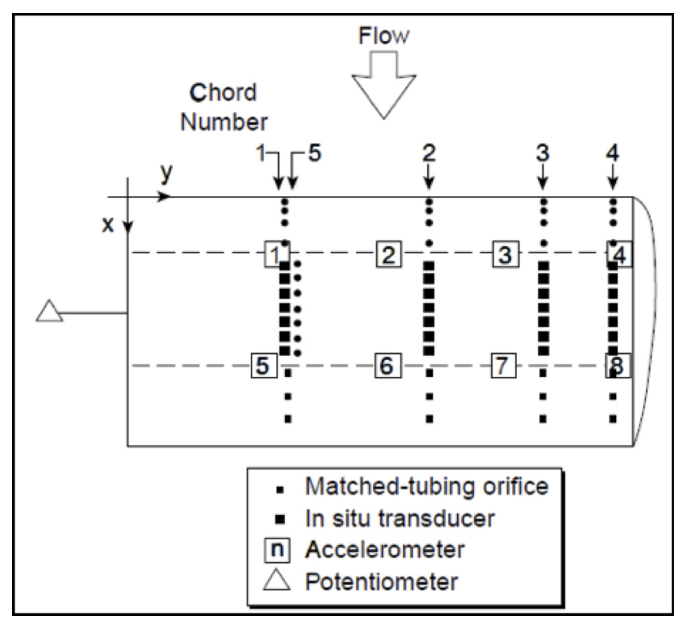

(b) RSW model instrumentation.

Figure 2. RSW model. 
Table 1. RSW analysis test cases.

\begin{tabular}{c|c|c|c|c|c|c}
\hline Test Case & Mach No. & $\begin{array}{c}\text { Mean } \alpha \\
(\mathrm{deg})\end{array}$ & $\begin{array}{c}\text { Pitch Osc. Freq. } \\
(\mathrm{f}, \mathrm{Hz})\end{array}$ & $\begin{array}{c}\text { Pitch Osc. Amp. } \\
(\theta, \mathrm{deg})\end{array}$ & $\begin{array}{c}\text { Reduced Freq. } \\
\omega \mathrm{c} / 2 V_{\infty}\end{array}$ & $\begin{array}{c}R_{c} \\
* 10^{6}\end{array}$ \\
\hline \hline 1 & 0.825 & 2.0 & 0 & 0.0 & 0 & 4.0 \\
2 & 0.825 & 4.0 & 0 & 0.0 & 0 & 4.0 \\
3 & 0.825 & 2.0 & 10 & 1.0 & 0.152 & 4.0 \\
4 & 0.825 & 2.0 & 20 & 1.0 & 0.304 & 4.0 \\
\hline \hline
\end{tabular}

\section{II.B. Workshop preparation analyses}

As part of the geometry modeling and grid generation effort, members of the AePW Organizing Committee (OC) conducted preliminary steady-state computations on the RSW wing to evaluate necessary grid quality and solution convergence. These preliminary analyses were conducted using FUN3D and later were confirmed using CFL3D. These preliminary computations immediately uncovered an issue with the wing modeling. The initial computational model simply contained the wing only from the root of the splitter plate outboard, assuming the splitter plate to effectively behave like a plane of symmetry in the computational domain. This model is labeled "Model A" in table 2 and in figures 3 and 4. An examination of the pressures at the four wing stations showed that for the inboard-most stations, the shock was predicted to be much farther aft than in the experimental data. Upon seeing these results, the OC conducted a parametric study that investigated different combinations of modeling the wind-tunnel wall and splitter plate. Four additional geometries were investigated: wing with a splitter plate with a tunnel wall as the symmetry plane (Model B), wing with a splitter plate with a tunnel wall treated as a viscous surface (Model C), wing without a splitter plate with a tunnel wall treated as a viscous surface (Model D), and wing without a splitter plate and a tunnel wall as a symmetry plane (Model E). For the cases without the splitter plate, the RSW wing geometry was simply extruded inboard to the wind-tunnel wall, adding seven inches to the span of the wing.

The pressure coefficient contour plots for Models A-E are shown in figures 3a-e. Corresponding pressure coefficient line plots at four span stations are shown in figure 4. Clearly, the presence of the viscous tunnel wall shifts the shock towards the leading edge of the wing. The model of the wing with the viscous tunnel wall with or without the splitter plate produced the best results when compared with the experimental data. In an attempt to further improve the match between the computational and experimental data, the size of the computational domain upstream of the wing was reduced from $100 c_{r e f}$ to $50 c_{r e f}$ (Model F in table 2). This changed the thickness of the boundary layer upstream of the wing and did result in a better match between the computational and experimental data.

Table 2. RSW preliminary analyses test cases

\begin{tabular}{c|c|c|c}
\hline $\begin{array}{c}\text { Preliminary Model } \\
\text { Designation }\end{array}$ & $\begin{array}{c}\text { Configuration } \\
\text { Description }\end{array}$ & $\begin{array}{c}\text { Wing Span } \\
\text { (inches) }\end{array}$ & $\begin{array}{c}\text { Upstream Boundary } \\
\text { Location }\end{array}$ \\
\hline \hline A & Wing+Symmetry Plane Tunnel Wall & 48 & $100 c_{r e f}$ \\
B & Wing+Splitter Plate & 48 & $100 c_{r e f}$ \\
C & Wing+Splitter Plate+Viscous Tunnel Wall & 48 & $100 c_{r e f}$ \\
D & Wing+Viscous Tunnel Wall & 55 & $100 c_{r e f}$ \\
E & Wing+Symmetry Plane Tunnel Wall & 55 & $100 c_{r e f}$ \\
F & Wing+Viscous Tunnel Wall & 55 & $50 c_{r e f}$ \\
\hline \hline
\end{tabular}




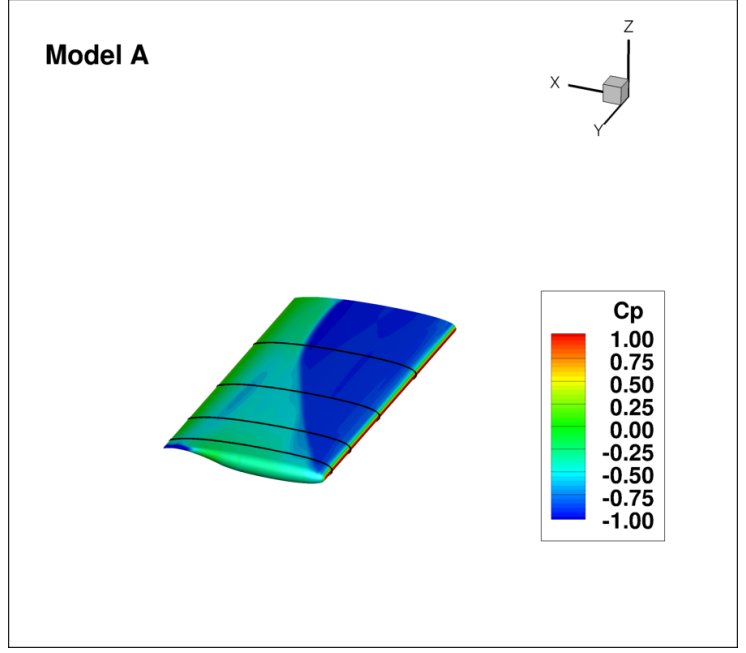

(a) Preliminary RSW Model A.

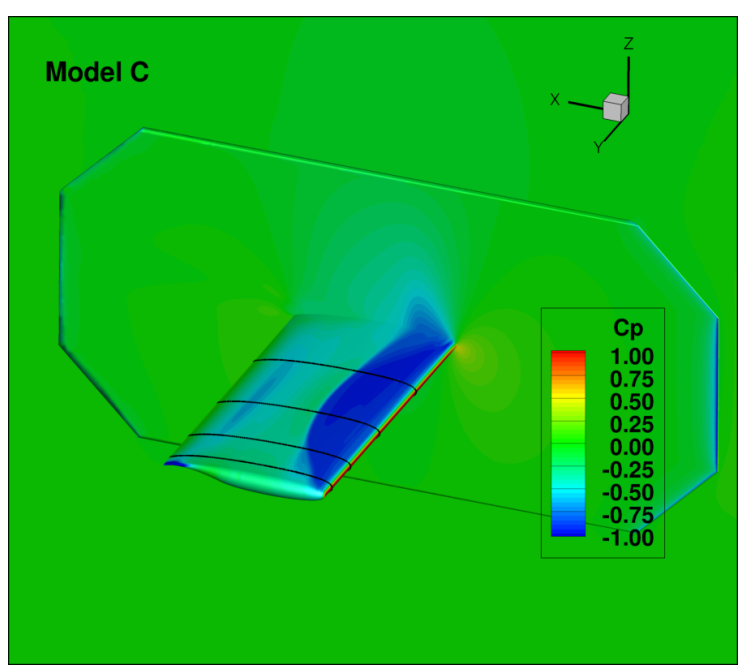

(c) Preliminary RSW Model C.

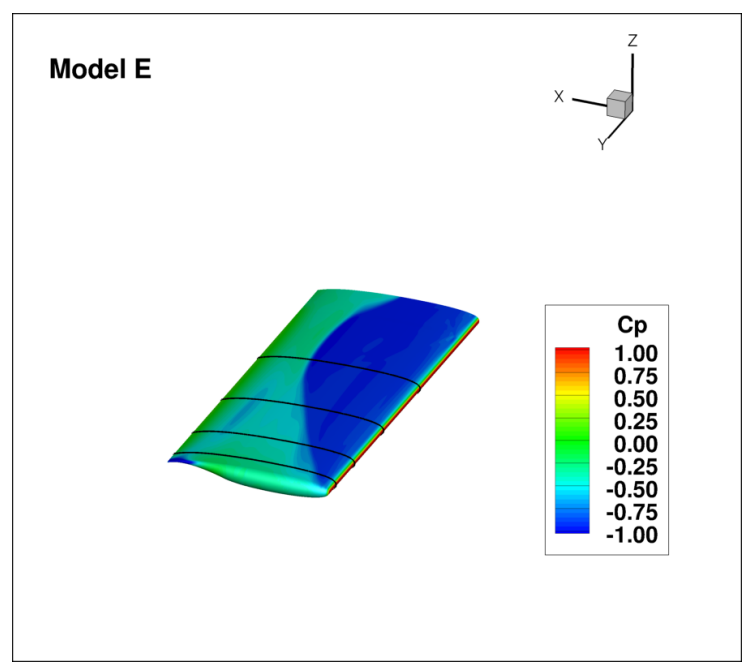

(e) Preliminary RSW Model E.

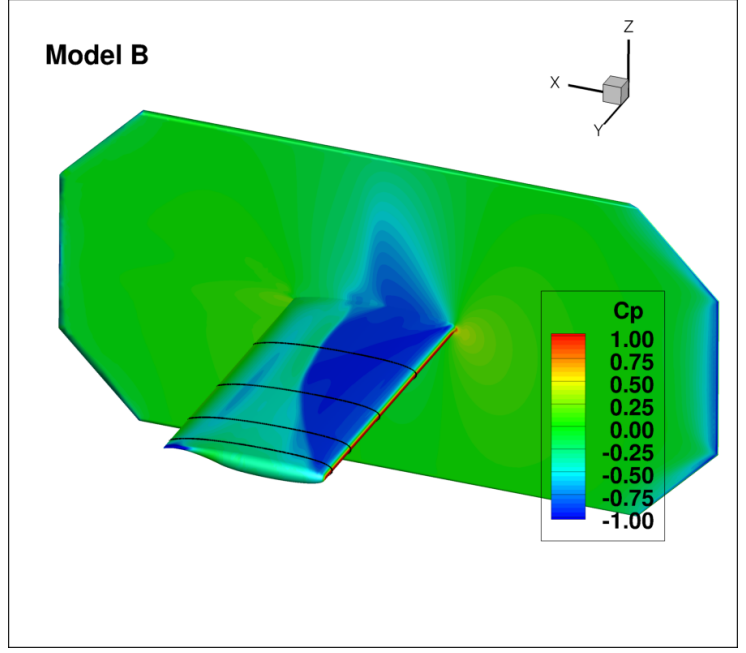

(b) Preliminary RSW Model B.

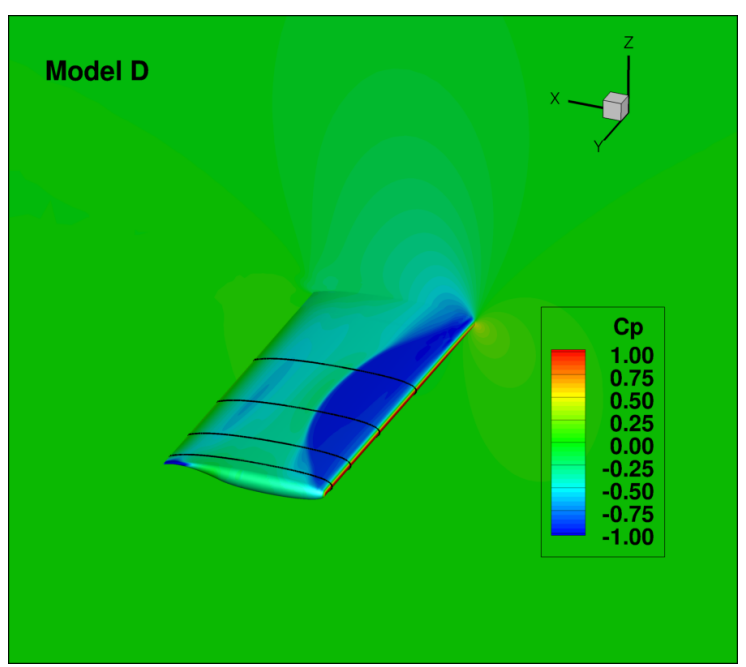

(d) Preliminary RSW Model D.

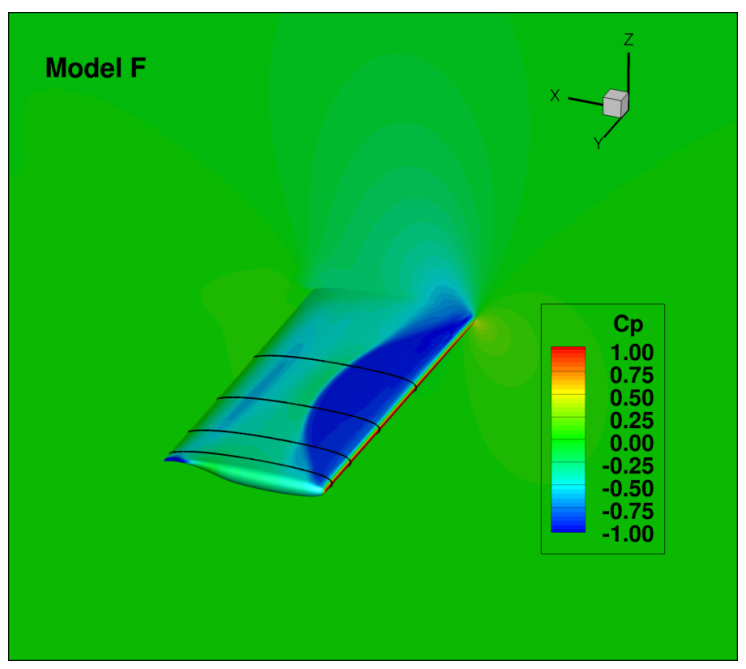

(f) Preliminary RSW Model F.

Figure 3. Preliminary CFD RSW analyses, $\alpha=2^{\circ}$. 


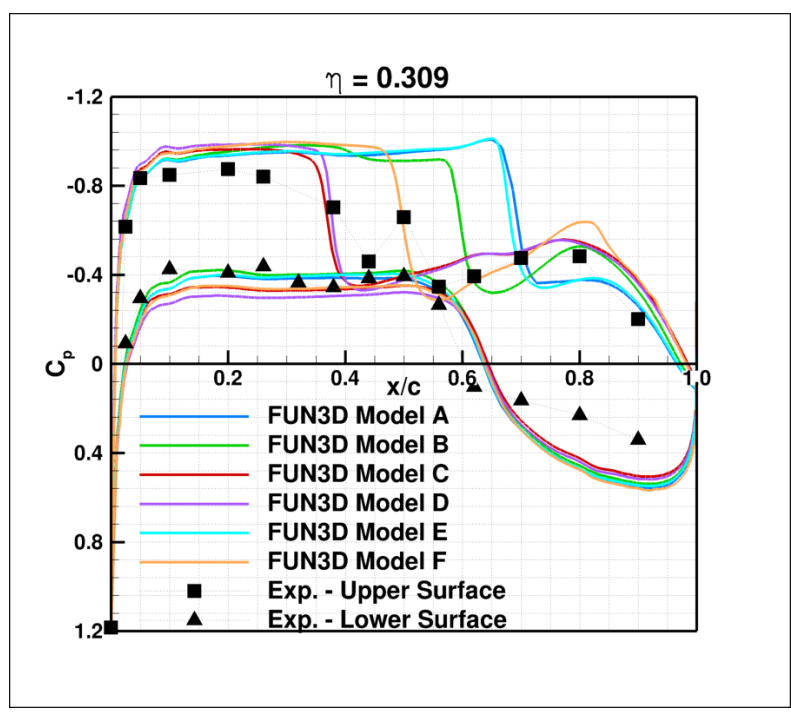

(a) Station $\eta=0.309$.

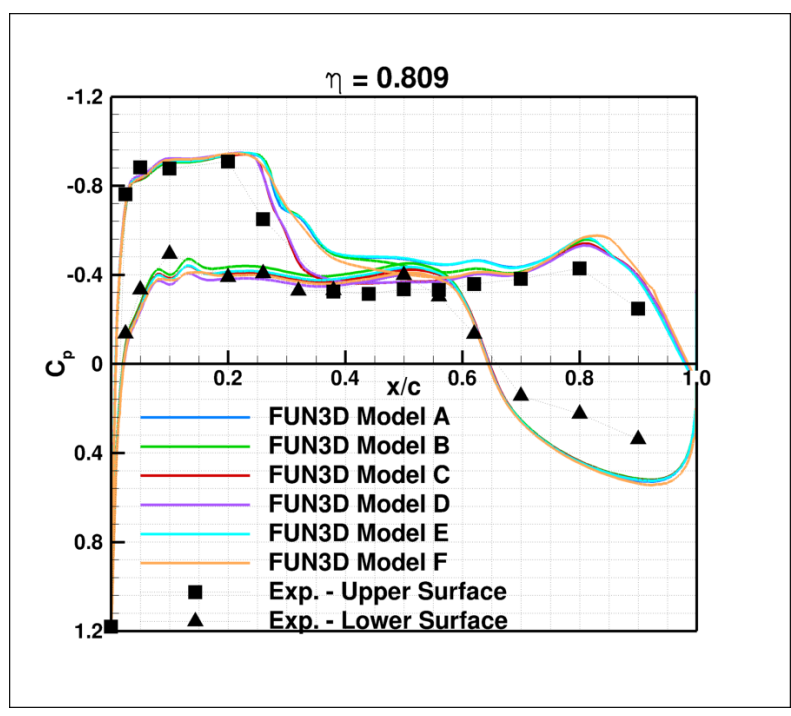

(c) Station $\eta=0.809$.

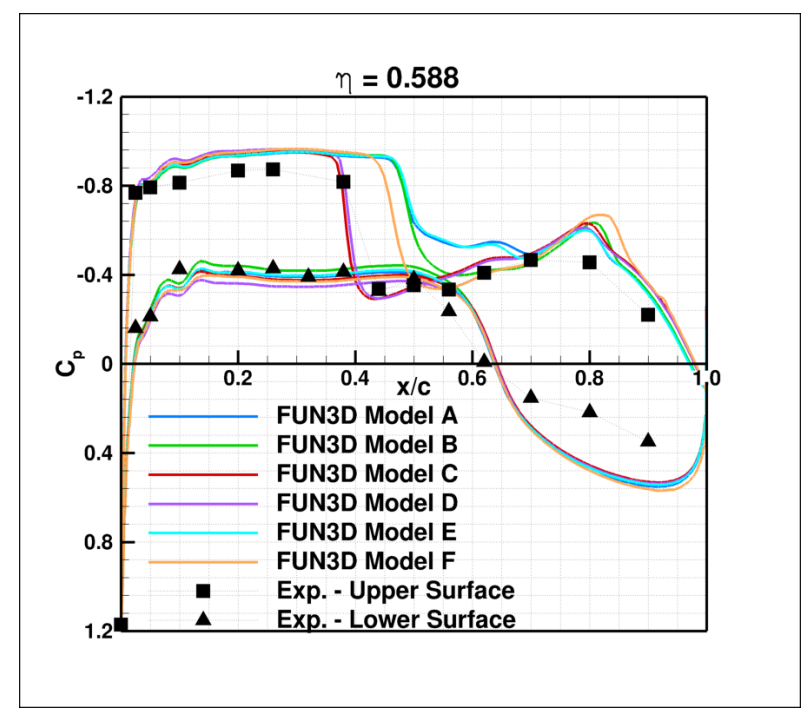

(b) Station $\eta=0.588$.

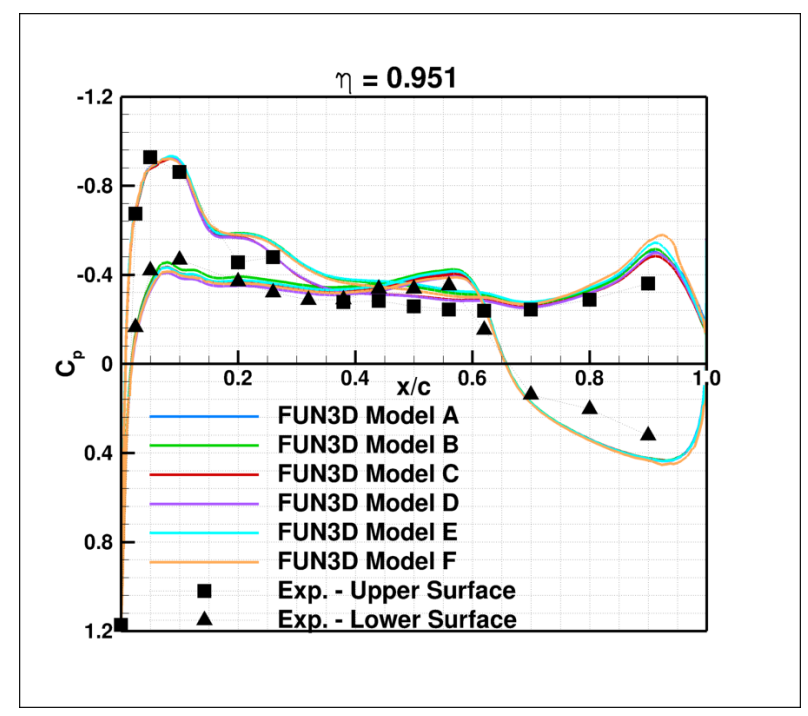

(d) Station $\eta=0.951$.

Figure 4. Experimental and computational pressure at four span stations, $\alpha=2^{\circ}$.

Preliminary computations were also performed using the CFL3D structured grid Reynolds-averaged Navier-Stokes code (RANS). In this analysis, the location of the upstream inflow boundary was determined based on prior knowledge of the experimental boundary-layer thickness $(\delta)$ and the displacement thickness $\left(\delta^{*}\right)$. Figure 5a shows the boundary layer $(\delta)$, displacement $\left(\delta^{*}\right)$, and momentum $(\theta)$ thicknesses measured in the TDT along the wall where the wing was mounted. ${ }^{13}$ Computationally, the location of the upstream boundary condition was varied until $\delta^{*}$ and $\delta$ matched the experimental data. Figure $5 \mathrm{~b}$ shows that the computed boundary layer profile and $\delta^{*}$ value at the $42 c_{\text {ref }}$ location of the upstream boundary condition (which corresponds to 1000 inches upstream of the wing) matched the experimental value from figure 5a. Figure 6 shows the computed pressure distribution for the wing with either a viscous tunnel wall or the tunnel wall treated as an inviscid plane, with the upstream boundary location at $42 c_{r e f}$. As with the FUN3D results, the change in shock location is dramatic, even though the lower surface pressure and the pressure ahead of the shock are only lightly affected by the presence of the viscous wind-tunnel wall. Based on the CFL3D analyses, FUN3D Model F with the upstream boundary relocated to $42 c_{r e f}$ was adopted for the AePW. 


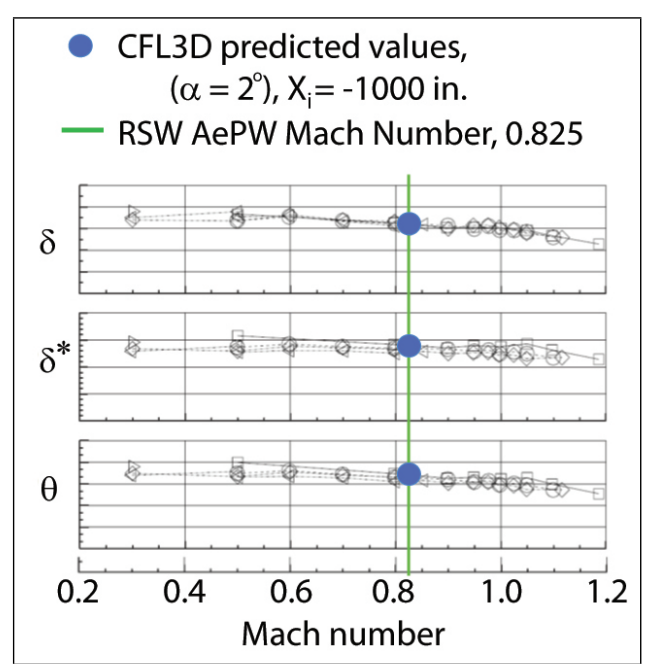

(a) TDT east wall experimental boundary layer parameters.

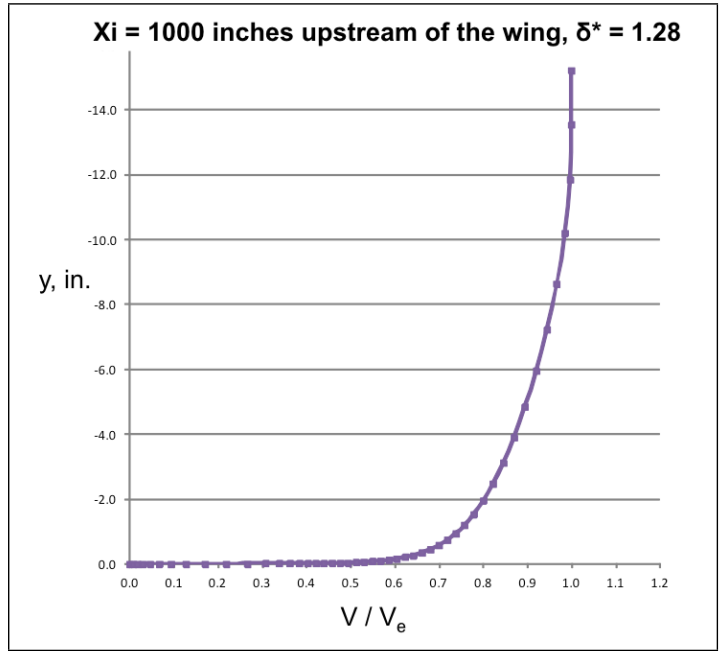

(b) CFL3D computed boundary-layer profile.

Figure 5. Experimental boundary-layer parameters (boundary layer $(\delta)$, displacement $\left(\delta^{*}\right)$, and momentum $(\theta)$ thicknesses) and computed displacement thickness $\delta^{*}$.

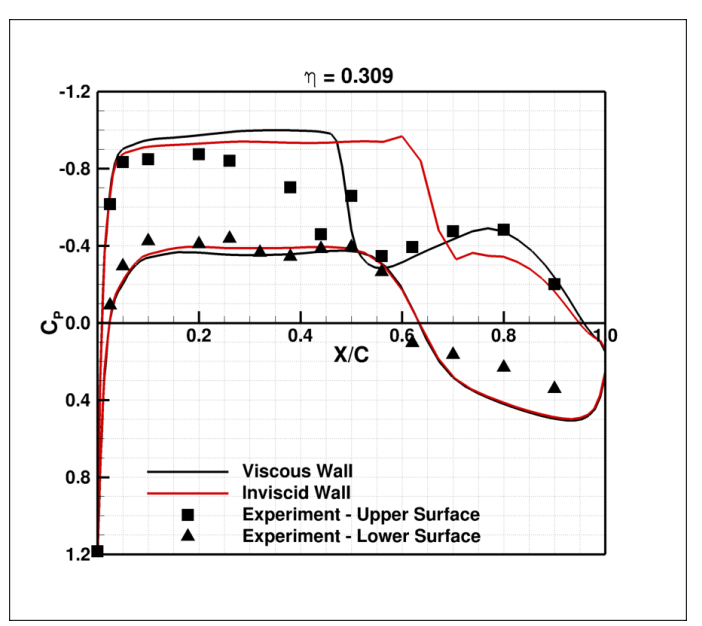

(a) Station $\eta=0.309$.

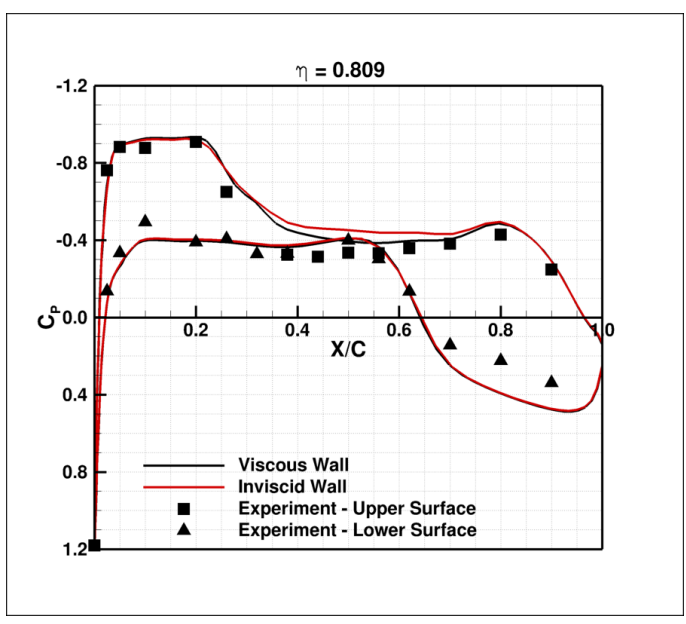

(c) Station $\eta=0.809$.

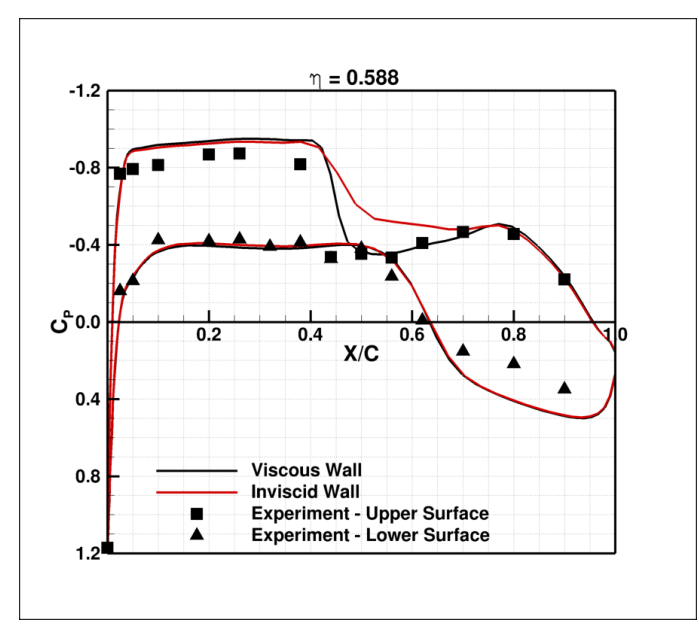

(b) Station $\eta=0.588$.

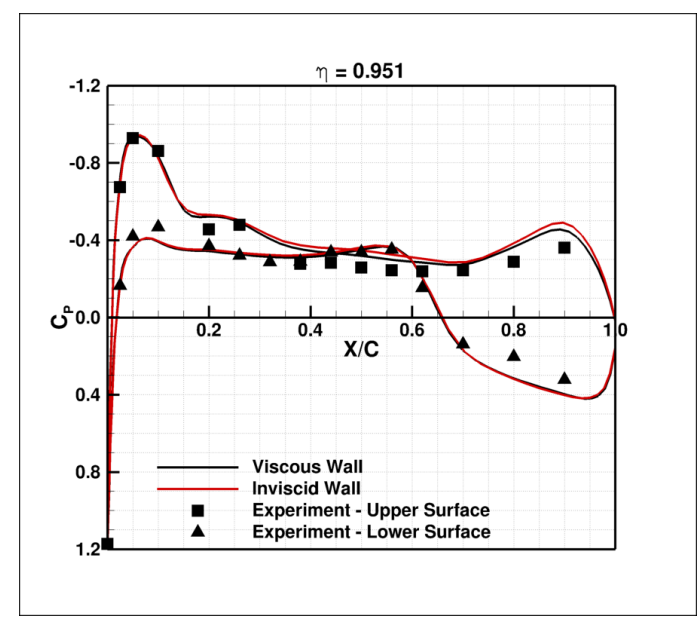

(d) Station $\eta=0.951$.

Figure 6. Experimental and computational (CFL3D) pressure at four span stations with upstream boundary at $42 c_{r e f}$. 


\section{II.C. Comparison quantities}

For the AePW and within this paper, comparisons between computational results and experimental data are made using different quantities for the unforced cases and the forced oscillation cases. The unforced cases are compared primarily using the time-averaged (mean) values of the pressure coefficients for the experimental data sets and the steady-state pressure coefficients obtained from the computations. The forced oscillation cases are compared primarily using frequency response functions (FRFs) of the pressure coefficients due to angle-faattack forcing functions. In this paper, the FRFs are examined as magnitude and phase characteristics.

Computational results are also compared with each other, examining the time histories of the pressure coefficients from the forced oscillation solutions and the integrated coefficients from the steady-state solutions. The RSW experimental data set consists only of reduced data, i.e., there are no time histories available. Additionally, there was no balance employed during the testing, so there are no force measurements to compare with the integrated coefficients obtained from the computational results.

\section{II.D. Features of a supercritical airfoil pressure distribution}

Throughout this paper, we discuss the pressure distribution of the RSW. The RSW airfoil has a 12\%-thick supercritical shape with a 2-dimensional design Mach number of 0.8 and a design lift coefficient of 0.6. ${ }^{11}$ The aerodynamic features associated with a supercritical airfoil are presumably well-known, at least for an unforced system. ${ }^{15-18}$ Harris describes these features for a supercritical airfoil near the design condition using figures reproduced here in figure 7.

The upper-surface pressure and related velocity distributions are characterized by a shock location significantly aft of the midchord, an approximately uniform supersonic velocity from about 5 percent chord to the shock, a <near sonic $>$ plateau in the pressure distribution downstream of the shock, a relatively steep pressure recovery on the extreme rearward region, and a trailing-edge pressure slightly more positive than ambient pressure. The lower surface has roughly constant negative pressure coefficients corresponding to subcritical velocities over the forward region and a rapid increase in pressure rearward of the midchord to a substantially positive pressure forward of the trailing edge.

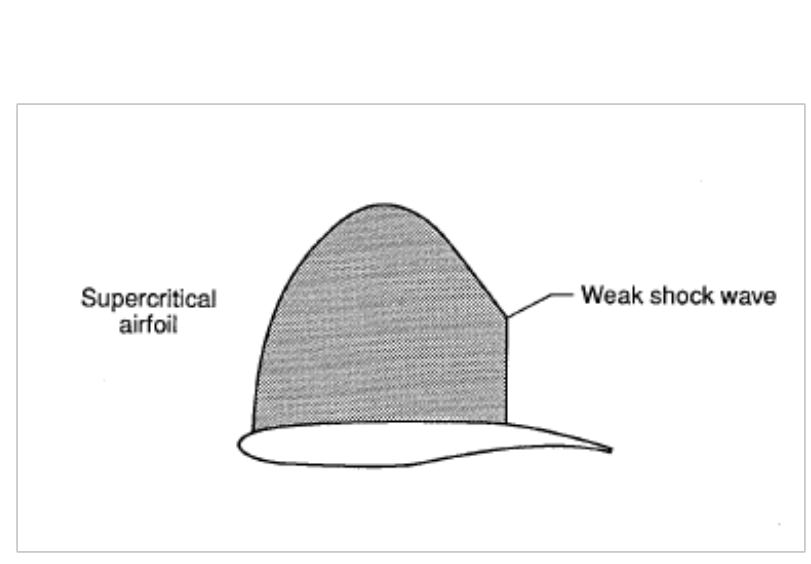

(a) Supercritical airfoil.

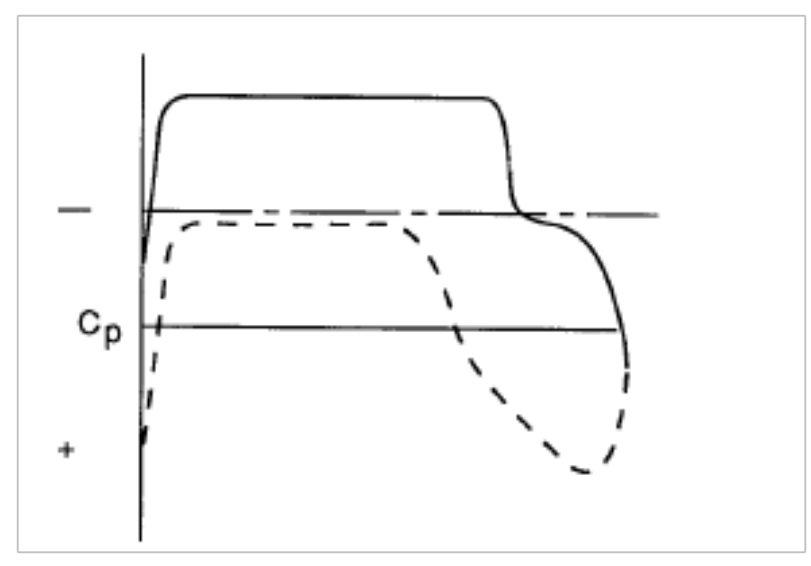

(b) Associated pressure coefficient distribution.

Figure 7. Flow fields and pressure coefficient distribution around a supercritical airfoil. ${ }^{15}$

The RSW computational and experimental pressure coefficient distributions for the $2^{\circ}$ angle-of-attack unforced system at the second span station are shown in figure 8 . These pressure distributions exhibit the features described above. At Mach $0.825, \alpha=2^{\circ}$, this airfoil is slightly above its design condition. Note that the mean value of the lift coefficient from all submitted computational results for the $2^{\circ}$ case is 0.57 , very near the published design lift coefficient of 0.6.

For the RSW unforced system, the principal features that need to be resolved as described above are the shock location and strength, the pressure plateau aft of the shock, the level of the supersonic plateau ahead of the shock, and the character of the flow in the lower surface cusp region. Based on (1) the fact that the mean pressure distribution resembles the design condition distribution for a supercritical airfoil and (2) other characteristics that will be discussed 
subsequently, prediction of trailing edge separation does not appear to be a requirement for the RSW, as will also be discussed later in this paper.

The forced oscillation data features parallel these characteristics, except that both strength (magnitude) and phasing need to be considered. These features include the oscillatory behavior of the shock, the aft pressure plateau, the supersonic plateau, and the suction peak. In the cusp region and just behind the shock, it is also possible that the dynamic characteristics include oscillations of flow separation and reattachment. Each of these features will be examined using the frequency response function representations of the forced oscillation data sets.

\section{II.E. Dynamic content of unforced system data}

The three configurations posed for the AePW were originally chosen to have matching steady and unsteady data sets for each configuration. Over the course of analyzing the experimental data sets, it became evident that not

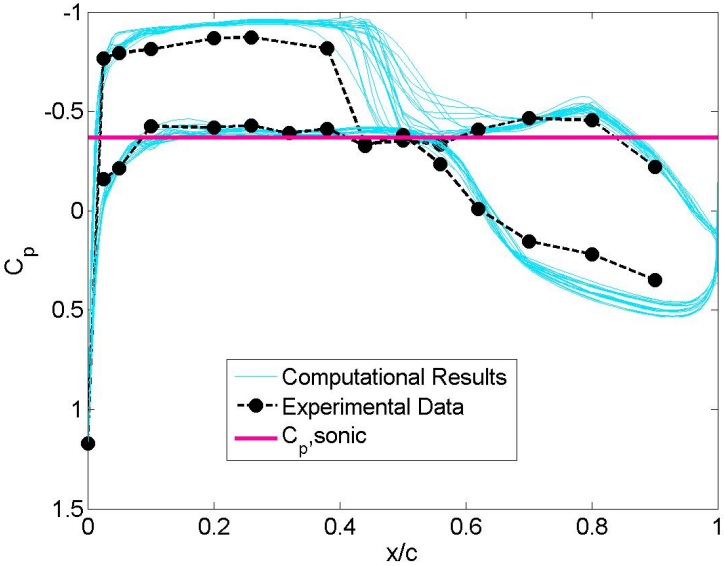

Figure 8. Mean pressure coefficient distributions, $\alpha=2^{\circ}$, Station 2 all of the experimental data was well-represented by a mean value. There were dynamics in the experimental data sets that were potentially resulting in misrepresentations of the system characteristics. Based on analysis of the BSCW time history data, it is suspected that the RSW experimental data may not correctly capture the pressures that would be associated with data obtained in a truly steady situation. The presence of shock motion in a presumed static data set can have the following effects on the resulting mean pressure distribution: smearing the shock over several chord stations, reducing the magnitude of the shock, and canting the shock towards the leading edge. This issue is being investigated through additional experimental data processing and additional computations using both the BSCW and the HIRENASD configurations. While the RSW time history data is not available, there is no reason to think that this case would be exempt from these effects.

From a terminology standpoint, we are using the term "unforced system" in this paper to identify the data sets that have traditionally been called "steady" or "static." We are also using the term "forced system" to identify the data sets that have previously been called "unsteady" or "dynamic."

A more thorough discussion of this discussion is presented. The experimental unforced system information presented in this paper is described using mean values. A mean value only adequately represents a time history data set if that time history has a symmetric distribution. In general, the mean is used to represent data that is thought to be Gaussian distributed, although the case of pure oscillations, the resulting distribution is symmetric so the mean is a reasonable quantity to represent the information. In the case of an oscillatory shock, however, the pressure measured by a sensor or calculated at a grid point in the spatial range of the oscillation can have a highly skewed distributionnot symmetric and poorly represented by a mean value.

As the shock moves across the sensor or grid point, the pressure value changes from the supersonic plateau (ahead of the shock) to the subsonic and possibly separated region of the distribution (aft of the shock), creating pressure floors and ceilings. The resulting time history appears nonlinear for any single spatial location, and when analyzed as a histogram, results in a highly skewed distribution. This was observed in the experimental data of the BSCW configuration, but it appears in the time history data from the RSW computational results as well. The potential impacts of the mean-value representation were principally assessed by examining the experimental data from the BSCW data set time histories.

If a feature-such as a shock-is moving rather than stationary, it can appear smeared over several chord stations, as the mean value represents an averaged position. Because the mean will also represent an averaged magnitude, the overall magnitude of the shock will likely be reduced relative to the actual supersonic plateau pressure that would otherwise be observed just ahead of the shock. Because the pressure magnitude is higher ahead of the shock than behind the shock, averaging the pressures together as the shock moves across a sensor or a grid point can result in the shock slanting towards the leading edge, rather than being more vertical.

In performing experiments where the static pressure distribution is desired, it is recommended that time history data be obtained, with time record lengths sufficient for identifying potential dynamic phenomena. Data should not be assumed to be Gaussian distributed. The time history data should be examined and represented by a first moment 
other than the mean if the histogram is not symmetric. Using the median has been suggested. Including upper and lower bounds is useful regardless of which first moment is used. They can provide a better representation of a static distribution than using the mean value. Time-lapse (smear) plots are useful for the same reason as the bounds, but they give additional insight into neighboring data point relationships.

\section{Workshop analyses}

Six analysis teams, shown in table 3, performed computations on the RSW for the AePW. Each team has a letter designation that will be used throughout this document in describing their analyses and results. The flow solver codes and choices made by each of the six teams are provided in table 4. All teams performing computations on the RSW configuration chose to use RANS flow solvers. The most popular choice of turbulence model was Spalart-Allmaras $(\mathrm{SA})^{19}$ in several varieties, with only one participant deviating from this choice and employing Menter's Shear Stress Transport (SST) model. ${ }^{20}$

Although the AePW OC generated and provided structured and unstructured grids, the workshop participants overwhelming chose to generate and use their own grids. Incompatibility of formats and files was cited as the main reason. The RSW grids used by the different analysis teams are detailed in table 5. The numbers of grid points that defined coarse (C), medium (M) and fine (F) grid resolutions for each analyst are listed in table 5 and shown in figure 9. Throughout this paper, grid factor, defined as the number of grid points or cells to the $-2 / 3$ power $\left(N^{-2 / 3}\right)$ is used to differentiate the grids. A plot of the RSW grid factors for each grid resolution is shown in figure 10.

For each AePW configuration, unforced system (steady) and forced oscillation (unsteady) analyses were performed and compared with existing experimental data sets. The RSW was treated as a rigid system, with the unforced system analysis being performed without consideration of any static aeroelastic properties. The forced oscillation cases were analyzed with time-accurate solutions for the unsteady flow fields.

Table 3. RSW analysis teams, affiliations and AePW designations

\begin{tabular}{ccc} 
Affiliation & Analysis Team Members & AePW Designation \\
\hline \hline RUAG Aviation & $\begin{array}{c}\text { Alain Gehri, } \\
\text { Daniel Steiling }\end{array}$ & $\mathrm{A}$ \\
\hline NASA & Pawel Chwalowski & $\mathrm{B}$ \\
\hline NASA & $\begin{array}{c}\text { David Schuster, } \\
\text { Andrew Prosser }\end{array}$ & $\mathrm{C}$ \\
\hline ANSYS Germany GMBH & $\begin{array}{c}\text { Thorsten Hansen, } \\
\text { Angela Lestari }\end{array}$ & $\mathrm{D}$ \\
\hline University of Wyoming & $\begin{array}{c}\text { Dimitri Mavriplis, } \\
\text { Mike Long, } \\
\text { Zhi Yang, } \\
\text { Jay Sitaraman }\end{array}$ & $\mathrm{E}$ \\
\hline University of Liverpool & Sebastian Timme & $\mathrm{F}$ \\
\hline \hline
\end{tabular}

\section{Convergence studies}

Each of the AePW analysis teams was asked to perform a grid convergence study for the RSW, performing computations on a coarse, medium, and fine grid as described in table 5 and figure 9 . Lift, drag, and pitching moment coefficients were computed for each of the grids and included in the workshop data submission package. The AePW organizers provided guidelines for reference area, reference length, and moment reference center to be used in the computation of the coefficients. Unfortunately, the previously discussed change in wing span due to the inclusion of the wind tunnel wall boundary layer in the analysis led to some confusion as to the correct wing span over which to integrate the loads, as well as how to deal with the change in wing planform area with respect to the provided reference area. Some analysts only modeled the wing from the splitter plate outboard, despite the recommendation to model the wing all the way to the wind tunnel wall. Couple this with the fact that no experimentally measured loads data is 
Table 4. AePW RSW flow solution information

\begin{tabular}{|c|c|c|c|c|c|}
\hline $\begin{array}{c}\text { Analysis } \\
\text { Team }\end{array}$ & $\begin{array}{l}\text { Software } \\
\text { Name }\end{array}$ & $\begin{array}{c}\text { Turbulence } \\
\text { Model }^{*}\end{array}$ & $\begin{array}{c}\text { Flux } \\
\text { Construction }\end{array}$ & $\begin{array}{c}\text { Flux } \\
\text { Limiter }\end{array}$ & $\begin{array}{c}\text { Oscillatory } \\
\text { Solution } \\
\text { Method }\end{array}$ \\
\hline $\mathrm{A}$ & NSMB & SA & Unknown & None & Elastic+TFI \\
\hline $\mathrm{B}$ & FUN3D & SA & Roe & Venkat & Elastic \\
\hline $\mathrm{C}$ & CFL3D & SA & Roe & None & Modal+TFI \\
\hline $\mathrm{D}$ & ANSYS CFX & SST & $\begin{array}{c}\text { 2nd Order Upwind/ } \\
\text { Rhie Chow }\end{array}$ & $\begin{array}{c}\text { Barth \& } \\
\text { Jesperson }\end{array}$ & Diffusion Equation \\
\hline $\mathrm{E}$ & NSU3D & SA & $\begin{array}{c}\text { Matrix Artificial } \\
\text { Dissipation }\end{array}$ & None & Full Grid Motion \\
\hline $\mathrm{F}$ & PMBv1.5 & SA & Osher & $\begin{array}{c}\text { MUSCL+ } \\
\text { van Albada }\end{array}$ & Full Grid Motion \\
\hline
\end{tabular}

Table 5. AePW RSW submitted grids

Analysis Grid Element Solver Number of Nodes or Cells, (millions) Wing Tip $\mid$ Wing Span,

\begin{tabular}{cccc||c|c|c||c|c} 
Team & Type $^{*}$ & Type $^{\dagger}$ & Type $^{\ddagger}$ & Coarse & Medium & Fine & Model $^{\S}$ & inches \\
\hline A & Str & Hex & Cell & 3.38 & 9.91 & 27.0 & Revoln & 55 \\
B & Unstr & Mix & Node & 2.88 & 7.07 & 18.23 & Revoln & 55 \\
C & Str & Hex & Cell & 0.18 & 1.42 & 11.18 & Scarf & 55 \\
D & Str & Hex & Node & 1.91 & 5.89 & 15.42 & Revoln & $48^{\text {II }}$ \\
E & Unstr & Mix & Node & 2.87 & 7.07 & 18.28 & Revoln & 55 \\
F & SMB & Hex & Cell & 2.32 & 6.60 & 18.63 & Revoln & 55
\end{tabular}

${ }^{*}$ Structured (Str), Unstructured (Unstr), Structured MultiBlock (SMB)

${ }^{\dagger}$ Hexagonal (Hex), Mixed Hexagonal \& Tetrahedral (Mix)

¥ Cell-centered (Cell), Node-centered (Node)

$\S$ Model geometry surface of revolution (Revoln), Scarfed tip (Scarf)

II Modeled only from splitter plate outboard to wing tip

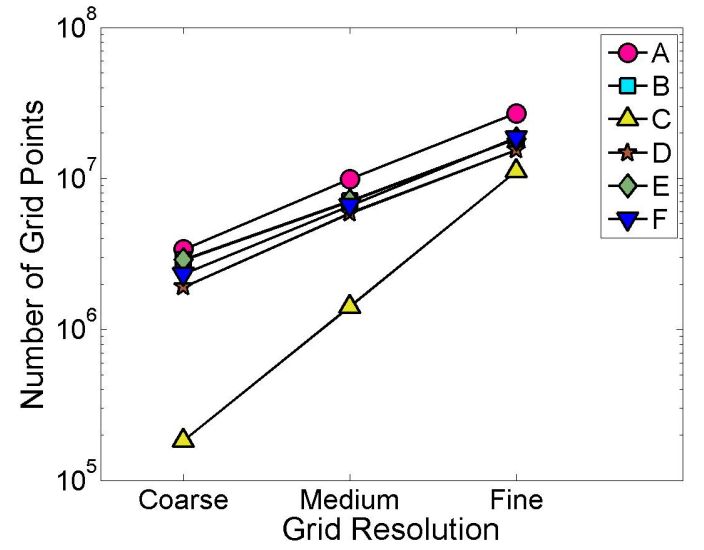

Figure 9. RSW grid resolutions for unforced analyses; Number of grid points.

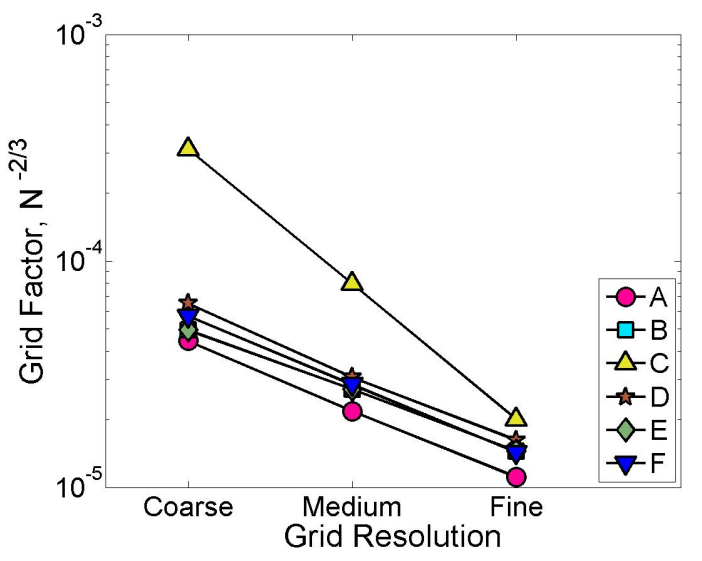

Figure 10. RSW grid resolutions for unforced analyses; Grid factor. 
available against which to anchor the computations for this case, and the suitability of these data for a definitive grid convergence study becomes questionable. Figure 11a plots the steady lift coefficient submissions from the analysts for the $\alpha=2^{\circ}$ case as a function of the number of grid points. All of the submissions appear to be converging to a fixed value, with some converging to this value from above, and some from below. Some computations appear to be better converged for a given grid refinement than others. There does not appear to be a consensus value towards which more than two methods are converging. Interestingly, there appears to be three values to which pairs of the analysts are converging. Analysts A and E seem to be converging towards a value in the 0.61 range, analysts $\mathrm{B}$ and $\mathrm{C}$ to a value in the 0.57 range, and analysts $\mathrm{D}$ and $\mathrm{F}$ to the 0.53 range. Despite the questions with regard to the potential procedural differences in computing the coefficients, it is encouraging that the computed fine grid lift coefficient varies by only approximately 0.08 across all the analysts. Similar data is available for the drag and pitching moment coefficients, as well as for the coefficient FRFs from the forced oscillation cases. But given that only qualitative conclusions can be reasonably derived from these data, they are not presented here.

To examine the accuracy of the solutions and to provide historical connection to the DPW and HiLiftPW, we followed the recommendation of the DPW community in defining a grid factor which is the number of grid points raised to the $-2 / 3$ power. Using the grid factor

...as a measure of the average cell size squared should produce straight-line plots for grid convergence studies based on second-order-accurate discretizations. ${ }^{21}$

The lift coefficient results shown in figure 11a are replotted in figure $11 \mathrm{~b}$ as functions of grid factor. With the exception of the results from analysis team E, to a first approximation the results in igure $11 \mathrm{~b}$ are linear, implying the respective solution methods are approximately second order accurate.

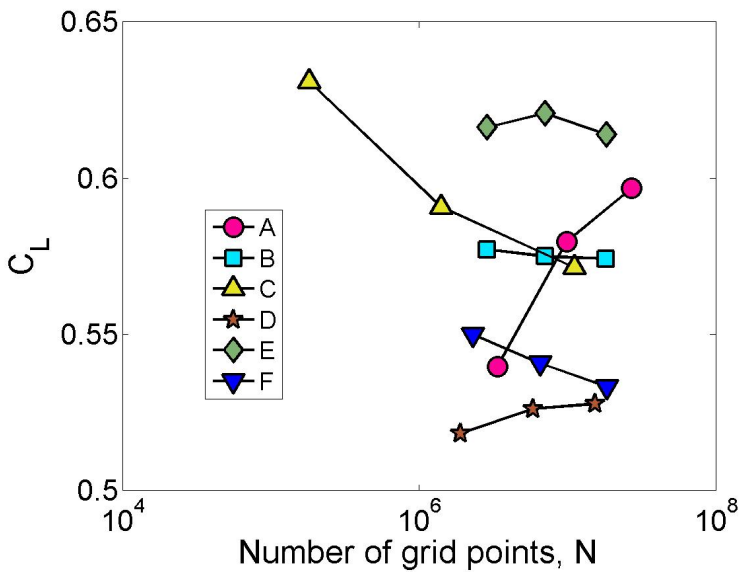

(a) Computed lift coefficient as a function of number of grid points.

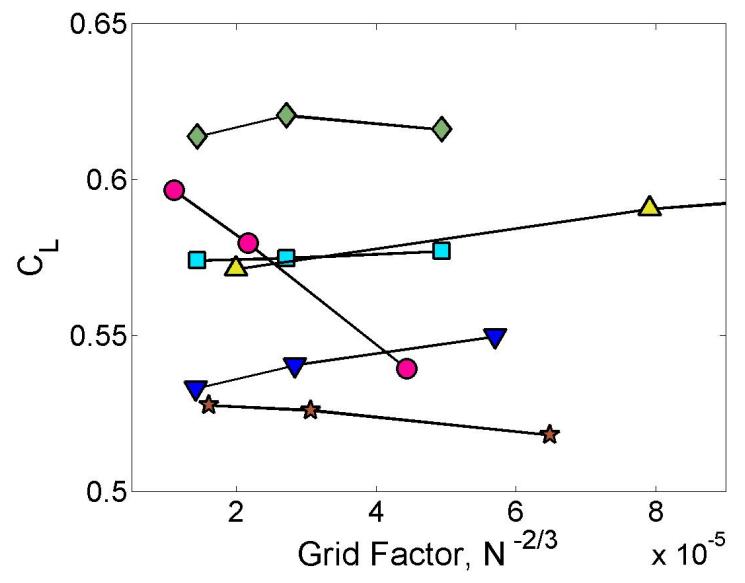

(b) Accuracy assessment, using lift coefficient and grid factor.

Figure 11. Grid convergence for unforced system response integrated coefficients, $\alpha=2^{\circ}$.

The analysts were also asked to consider the influence of time step size on their results. Only two of the six analyst submissions contained systematic variations in time step to assess convergence. Figure 12 shows the magnitude of the unsteady lift coefficient FRFs for the $10 \mathrm{~Hz}$ forced oscillation case submitted by the analysts. All analysts provided data at a single time step (64 time steps per cycle of motion), except for Analysts B and C who provided two and three different time steps, respectively. No definitive statement can be made about the temporal convergence characteristics for this case using these data. The three time steps provided by Analyst $\mathrm{C}$ are not monotonically converging towards a fixed value.

Subiterations between time steps were executed by all of the analysts, but each used their own scheme for determining the number of subiterations or subiteration convergence criteria. In retrospect, asking for convergence criteria information may have been beneficial in interpreting the data. In Cummings et a.1, ${ }^{22}$ the authors suggested that spatial and temporal resolution be simultaneously addressed. Having examined the solution processes used in the AePW analyses, it also appears relevant to consider the subiteration convergence criteria simultaneously.

The primary issue in not performing thorough time step convergence studies, much less performing simultaneous grid convergence studies, was one of computational time and the lack of an agreed-upon systematic approach to address combined spatial and temporal convergence. An important secondary issue was determining the parameters to 
examine for this type of convergence study, both in terms of response parameters and refinement parameters. For the data shown here, the magnitude of the total lift coefficient was chosen as the response parameter, but this isn't viewed as ideal. ${ }^{22}$ In computing the coefficients, the integration process removes some of the essential features of the flow field that should drive the time step convergence.

These data sets illustrate that the workshop goal of addressing time step convergence has not been satisfied for the RSW configuration. The proper approach to addressing this issue still remains to be investigated.

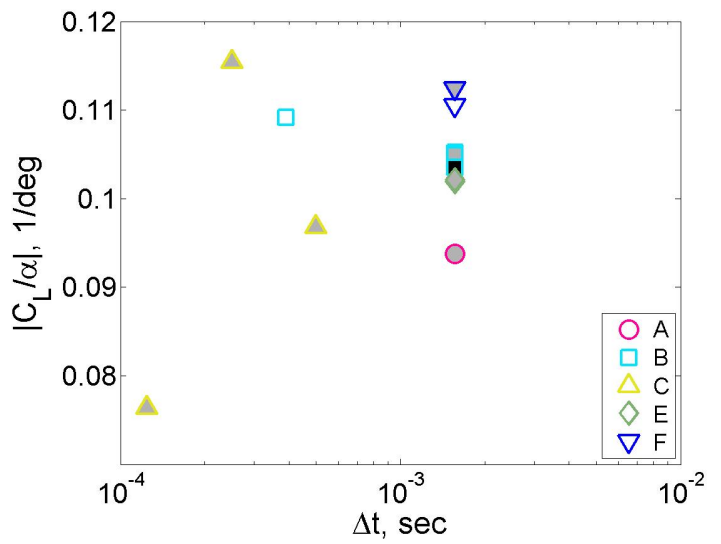

Figure 12. Time convergence for lift coefficient for $10 \mathrm{~Hz}$ forced oscillation case: (Coarse grids: white-filled symbols; Medium grids: grey-filled symbols; Fine grids: black-filled symbols).

\section{Mean pressure distributions}

The unforced system data was analyzed by computing the time-averaged (mean) value for each sensor (experimental data) or corresponding grid point (computational data). The resulting pressure coefficient distributions are presented in Appendices A and B. For each of the two unforced system test cases listed in table 1, there are eight plots in the appendix (upper and lower surfaces at 4 span stations). Each plot contains all of the computational results submitted for that test case. Results in this paper are generally shown with separate plots for the upper and lower surfaces, with the vertical axes inverted.

Example plots are shown for the upper and lower surfaces in figure 13, corresponding to $\alpha=2^{\circ}$ at the second span station, $\eta=0.588$. In the figure, data from each of the analysts is indicated using a different color. The different data sets from a given analyst are shown with a different symbol. The key for figure 13 is provided in figure 30 of Appendix A, showing the analysts' designation letters and the qualitative grid resolution descriptions. The features present in the mean data recognizably correspond to those identified for a typical supercritical airfoil near the design condition, presented earlier in figure 7.

For the purposes of discussing the pressure distributions at $2^{\circ}$, test case 1 , please refer to the plots in Appendix A. The shock strength is defined for these analyses as the change in the magnitude of the pressure coefficient across the shock, from the supersonic plateau ahead of the shock to the point at the foot of the shock or at the aft end of any shock-induced separation, as depicted using an example data set in figure 14a. The shock leading edge point was determined by finding the minimum $C_{p}$ value (labeled in the figure as Supersonic plateau peak) for a given data set and then searching aft along the chord for a slope exceeding a prescribed value. For the inboard span station, the shock trailing edge point was determined by finding the maximum $C_{p}$ value in the $20 \%$ of the chord aft of the shock leading edge location, and then searching forward along the chord to find a slope exceeding a prescribed value. For the other three span stations, the same algorithm was used, but $30 \%$ of the chord was included in the aft-of-shock search region. For cases where no appropriate slope was detected, the shock trailing edge location is not reported, but the maximum value of pressure coefficient was used to determine the shock strength. A mean pressure value was calculated from the pressures at the shock leading and trailing edge points. The data set was then searched for the two points straddling this mean pressure. Linear interpolation was applied using the chord locations and pressures of these straddling points to calculate the shock center location.

Beginning with the inboard span station, $\eta=0.309$, the mean pressure distribution on the lower surface and ahead of the shock on the upper surface show computational results that are relatively consistent with each other. However, the shock location predictions vary widely at this span station, figure 15a. The results can be grouped into two sets: 


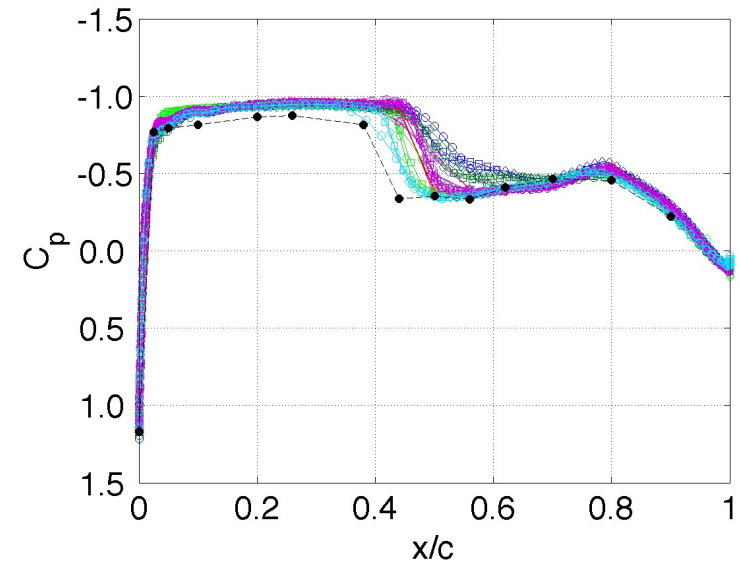

(a) Upper surface.

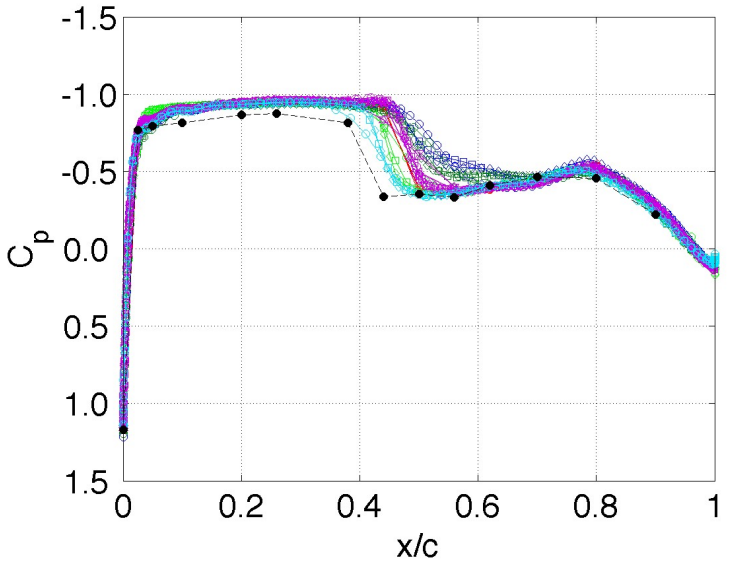

(b) Lower surface.

Figure 13. Unforced system mean data: Computational data sets (colors) submitted overlaid with experimental data (black): $\alpha=2^{\circ}$, Station 2 .

those predicting a forward shock location at approximately 50\% chord; and those predicting the shock further aft at approximately $65 \%$ chord. However, no modeling or analysis factor has been found to distinguish one grouping from the other. Figure 15 shows the shock center location and range predicted by each analysis team for each of the submitted computations, with each span station shown in a separate subfigure. The corresponding strength for each submitted data set is shown in figure 16, again with each span station in a separate subfigure.

It is interesting to note that although the pressure distribution at span station 3 looks benign by comparison with the more inboard stations, the shock ranges are more extensive chordwise. This is principally due to the shape of the pressure distribution just aft of the shock.

Treating the shock properties for all test case 1 computational results as a single data set, the mean and extreme values were calculated. These are shown in table 6 . All analysis teams submitted results for coarse, medium and fine grids for this case, as shown in figure 14. There were no distinctive trends in the results based on grid size; examination of the results shows that the variation introduced by analysis team appears larger than the variation introduced by grid refinement.

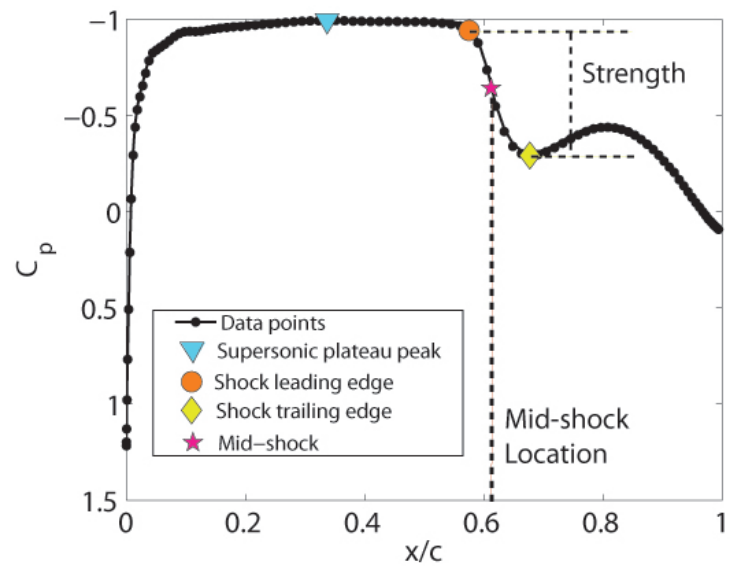

(a) Steady pressure coefficient of unforced system.

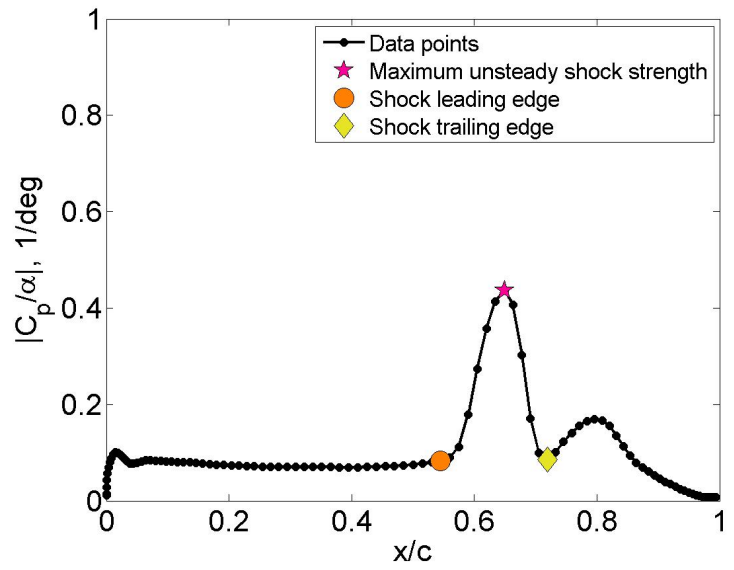

(b) Frequency response function magnitude of forced oscillation system.

Figure 14. Shock properties assessment example: Analysis Team A, $\alpha=2^{\circ}$, Station $1(\eta=0.309)$.

The computational results can also be compared with the experimental data using figure 15 and the plots shown in Appendix A. The shock center location for the experimental data is indicated by the black vertical bars in each of these plots. At the inboard span station (shown in figure 15a) the shock location appears to approximately agree with 


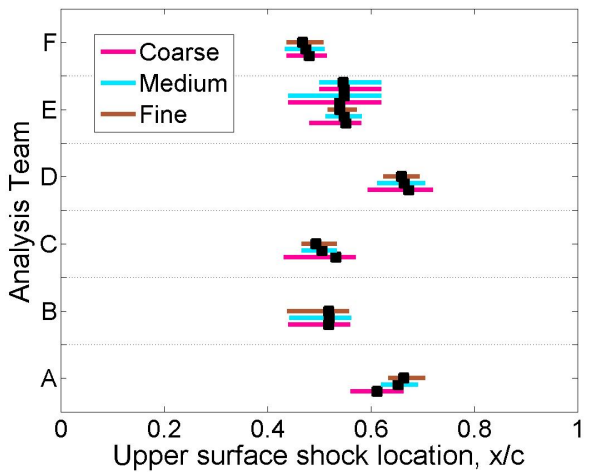

(a) Station 1 .

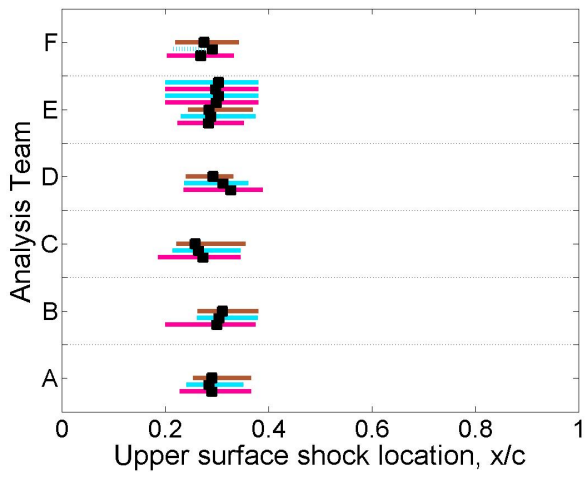

(c) Station 3 .

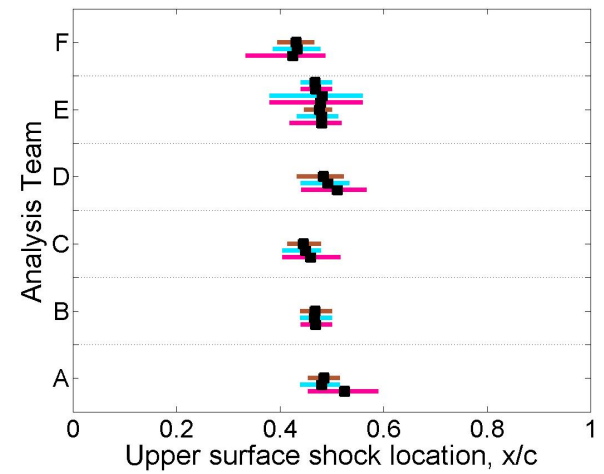

(b) Station 2.

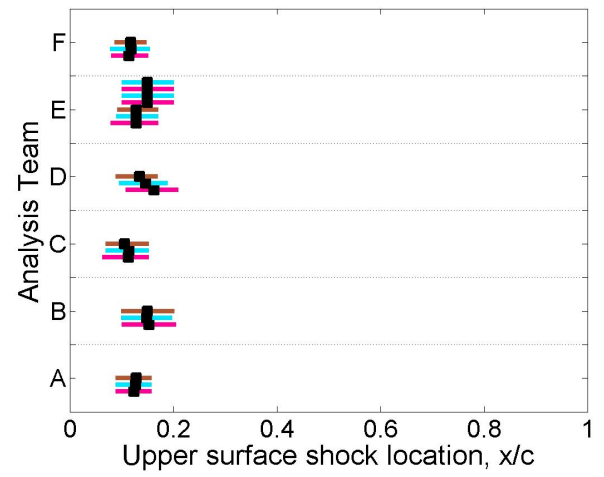

(d) Station 4 .

Figure 15. Upper surface shock center location (black filled symbols) and range (lines) for each set of computational results (Case 1: Unforced system at $\alpha=2^{\circ}$ ).

Table 6. Computational results, Upper surface shock properties, unforced system, $\alpha=2^{\circ}$

\begin{tabular}{cc|ccc|ccc|ccc} 
& & \multicolumn{3}{|c|}{ Location $(\mathrm{x} / \mathrm{c})$} & \multicolumn{3}{c|}{ Strength $\left(\left|\Delta C_{p}\right|\right)$} & \multicolumn{3}{c}{ Supersonic Plateau $\left(C_{p}\right)$} \\
\hline Station & $\eta$ & Mean & Min & Max & Mean & Min & Max & Mean & Min & Max \\
\hline \hline 1 & 0.309 & 0.56 & 0.43 & 0.72 & 0.66 & 0.58 & 0.78 & -0.99 & -1.03 & -0.95 \\
2 & 0.588 & 0.47 & 0.33 & 0.59 & 0.49 & 0.29 & 0.61 & -0.95 & -0.98 & -0.94 \\
3 & 0.809 & 0.29 & 0.19 & 0.39 & 0.43 & 0.36 & 0.54 & -0.94 & -0.96 & -0.91 \\
4 & 0.951 & 0.13 & 0.06 & 0.21 & 0.34 & 0.26 & 0.38 & -0.91 & -0.95 & -0.86
\end{tabular}




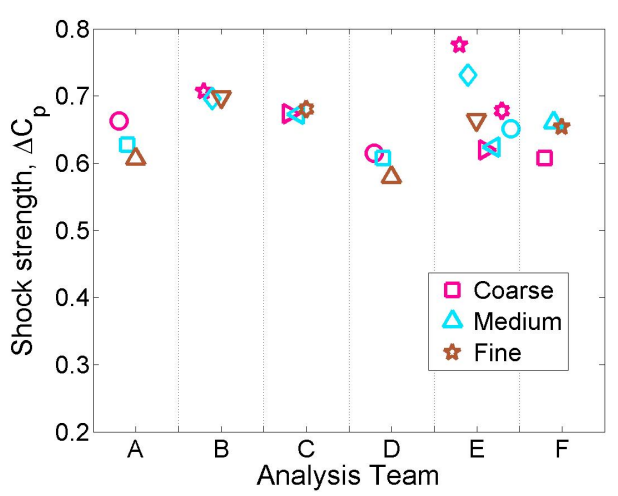

(a) Station 1.

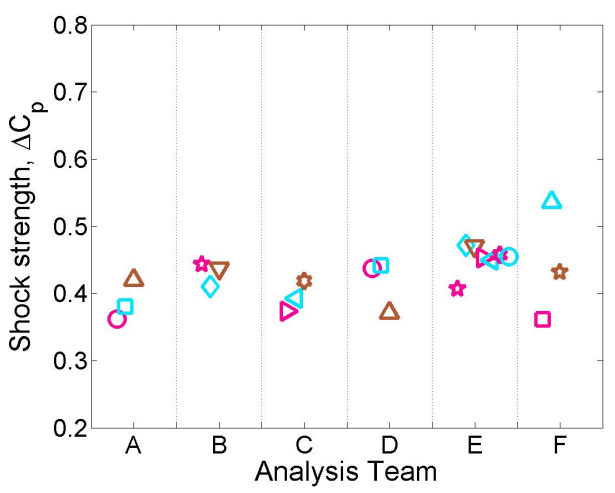

(c) Station 3 .

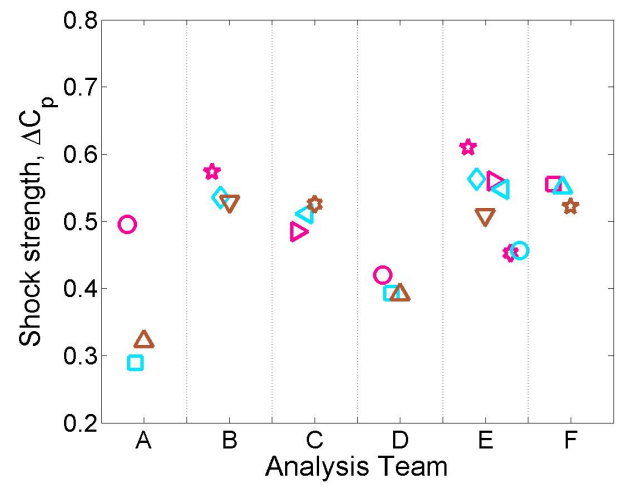

(b) Station 2.

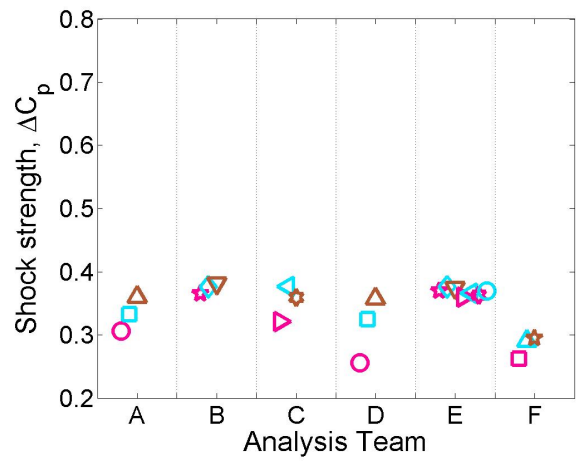

(d) Station 4 .

Figure 16. Shock strength generated for each set of computational results (Case 1: Unforced system at $\alpha=2^{\circ}$ ). 
the forward shock location predictions. The experimental upper surface pressure distribution, however, is complicated by a divot at $\mathrm{x} / \mathrm{c}=0.44$ which will be discussed later with regard to assessing sensor health. The supersonic plateau appears to be slightly overpredicted by all analysts, but the plateau aft of the shock appears well-predicted by those computations with the forward shock location. Going hand-in-hand with the shock being aft, the peak of the aft pressure hump is overpredicted in terms of magnitude and is predicted too far aft.

On the aft portion of the airfoil lower surface at the inboard station, none of the computational results compare well with the experimental data; the computations overpredict the pressure in this region. This is in the region of the supercritical airfoil cusp and beyond; it is speculated that the experimental data may contain geometry-induced separation in this region.

Moving outboard to the second span station, $\eta=0.588$, all computational models predict the shock further aft than is indicated in the experimental data. The computational results are a more tightly bunched data set than at the inboard span station. Here, the midpoint of the shock predictions range from $\mathrm{x} / \mathrm{c}=0.43$ to 0.54 . Several of the analyses, by teams A and D, show a very flat aft pressure plateau. On the lower surface, the general agreement of the computational results continues, and the discrepancy on the aft portion with respect to the experimental data also continues.

At the third span station, $\eta=0.809$, the influence of the wind tunnel wall boundary layer is diminished, and all of the computational results are qualitatively similar and also agree fairly well with the experimental data. The exception to this general statement is in the lower surface cusp region, where the computations continue to predict higher pressure coefficients than shown in the experimental data.

At the outboard span station, $\eta=0.951$, the mean pressure distributions are again tightly bunched, with a few exceptions. The coarse grid solution from analysis team D behaves differently between 15 and $20 \%$ chord on the upper surface and near the leading edge on the lower surface. Some of the results from analysis team $\mathrm{C}$ behave differently near the trailing edge of the upper surface and over the forward portion of the lower surface.

\section{V.A. Angle-of-attack effects}

The plots showing the mean pressure coefficient distributions for the $4^{\circ}$ angle of attack case are given in Appendix B. The same characterization methods used to generate the values shown in table 6 were applied to the submitted data for the $4^{\circ}$ angle of attack case, with the results shown in table 7 .

The plotted and tabulated $4^{\circ}$ data, in comparison with the $2^{\circ}$ data, shows a definite angle of attack effect. As the angle of attack increases, the shock moves aft. The magnitude of the pressure at the supersonic plateau increases with increasing angle of attack. The aft-of-shock pressure plateau hump becomes less pronounced (i.e. becomes flatter) as the angle of attack increases. The forward migration of the shock location as the tip is approached is maintained in this data set. The shock at all span stations is stronger than for the $2^{\circ}$ case, but the variation in the predicted shock strength has also grown. The supersonic plateau magnitude has increased relative to the data calculated for the $2^{\circ}$ case. These trends, observed in both the experimental data and the computational results, correspond to expected trends with increasing angle of attack for a supercritical airfoil at conditions without trailing edge separation.

Table 7. Computational results, Upper surface shock properties, unforced system, $\alpha=4^{\circ}$

\begin{tabular}{cc|ccc|ccc|ccc} 
& & \multicolumn{3}{|c|}{ Location $(\mathrm{x} / \mathrm{c})$} & \multicolumn{3}{c|}{ Strength $\left(\left|\Delta C_{p}\right|\right)$} & \multicolumn{3}{c}{ Supersonic Plateau $\left(C_{p}\right)$} \\
\hline Station & $\eta$ & Mean & Min & Max & Mean & Min & Max & Mean & Min & Max \\
\hline \hline 1 & 0.309 & 0.57 & 0.43 & 0.74 & 0.81 & 0.66 & 1.02 & -1.17 & -1.25 & -1.12 \\
2 & 0.588 & 0.54 & 0.41 & 0.66 & 0.73 & 0.61 & 0.83 & -1.12 & -1.18 & -1.11 \\
3 & 0.809 & 0.34 & 0.23 & 0.44 & 0.53 & 0.43 & 0.64 & -1.11 & -1.15 & -1.09 \\
4 & 0.951 & 0.14 & 0.07 & 0.24 & 0.38 & 0.30 & 0.42 & -1.08 & -1.12 & -1.03
\end{tabular}

\section{V.B. Separated flow assessment}

The ideal supercritical airfoil design was envisioned to be shock-free, but enforcing this resulted in inefficiencies at off-nominal conditions, so supercritical airfoils generally have weak upper surface shocks. ${ }^{15}$ The supercritical airfoil design process involved shaping the pressure distributions, with one objective being to control boundary layer separation. The supercritical airfoil designers measured separation boundaries for their airfoils, delineating separation onset conditions as Mach-normal force combinations, exemplified by figure 3 from Harris. ${ }^{15}$

The likelihood of shock-induced separation increases with the shock strength. A weak shock thickens the boundary layer; a stronger shock locally separates the boundary layer followed by reattachment; a very strong shock can separate 
the boundary layer to the trailing edge. ${ }^{23}$ Data for the upper surface of the wing is examined for indications of shockinduced separation at the trailing edge and locally aft of the shock. Additionally, the lower surface data is examined for indications of geometry-induced separation in the region of the cusp.

In studying the separation tendencies of the RSW, it should be remembered that only the reduced data is available. Potential insights that might be gained from time history data are lost. If the time history data were available, we could plot histograms of the pressures in different regions of the unforced system data, as well as the coherence of the frequency response functions. Both of these assessments might provide insight into the likelihood of the flow being separated. Further discussions of these assessment tools are postponed to future publications focused on the BSCW and HIRENASD data sets, where time histories are available.

\section{V.B.1. Trailing edge separation}

The experimental data sets at the previously discussed test conditions are plotted together to examine the angle-ofattack effects with respect to assessing flow separation in figure 17 . In addition to the data at $2^{\circ}$ and $4^{\circ}$, there is a data set available at $\alpha=0^{\circ}$, which is also shown in the figure.

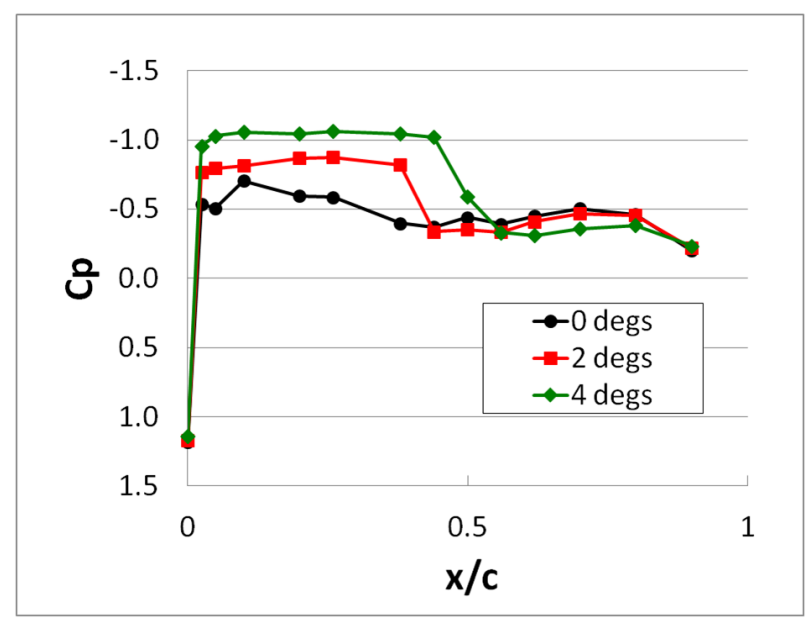

(a) Upper surface.

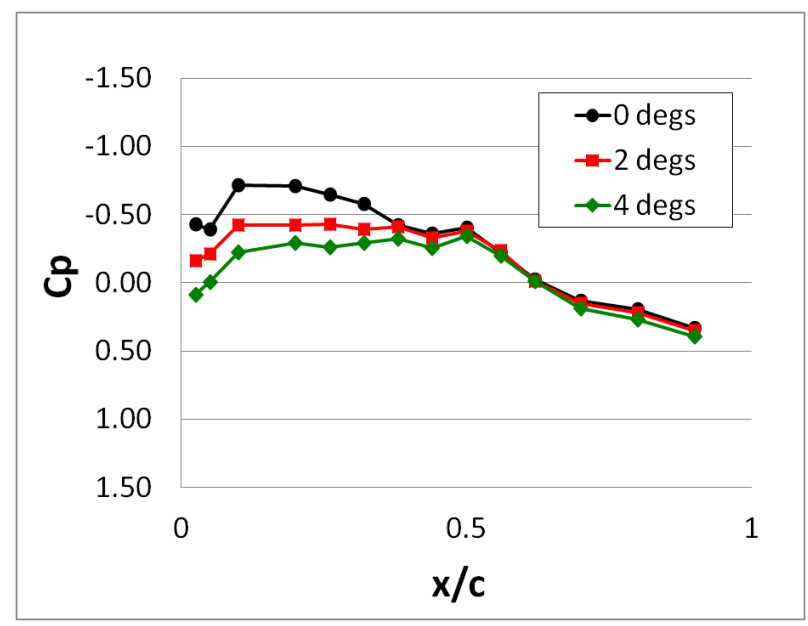

(b) Lower surface.

Figure 17. Experimental data; Station 2; Variation of mean pressure coefficient with angle of attack.

Comparisons of the pressure distribution with known separated flow cases were used to assess whether separation was likely to have occurred at the trailing edge or aft of the shock. The trailing edge pressure is the key indicator for separated flow. If the pressure coefficient on the upper surface is positive, the flow will generally be attached. For all RSW cases, the pressure coefficient for the last sensor or the last grid point is positive. Separated flow would also cause the trailing edge pressure distribution on the upper to change with angle of attack. ${ }^{15}$ This is not observed in either computations or experiment. Additionally, the value of the pressure coefficient at the trailing edge in all cases (computational and experimental data for all angles of attack) is positive. Examining the pressure distribution shape aft of the shock, it resembles the pressure distribution of a supercritical airfoil near the design condition. Separated flow causes the plateau in this region to disappear and be replaced by a uniformly downward sloping distribution. This is particularly well-illustrated by Hillenherms et al. ${ }^{24}$ They present a shadowgraph and associated pressure distribution for a supercritical BAC 3-11/RES/30/21 airfoil. The shadowgraph clearly shows aft-wing separation, and the pressure distribution has a monotonic aft-of-shock slope.

\section{V.B.2. Local shock-induced separation}

The guideline for assessing the shock strength is based on local Mach number. A local Mach number above 1.3 indicates that the shock should be classified as a "strong" shock, implying that the boundary layer is likely to separate at least locally just aft of the shock. ${ }^{25}$ The local Mach number can be computed from the Cp level (specified here by the minimum value of $\mathrm{Cp}$ ), the freestream Mach number (0.825), and the ratio of specific heats for the test medium 
$\left(\gamma_{R-12}=1.14\right)$ using equation $1 .^{26}$

$$
M_{\text {local }}=\sqrt{2 /(\gamma-1) \frac{1+\frac{\gamma-1}{2} M_{\infty}^{2}}{\left(C_{p, \text { local }} \gamma M_{\infty}^{2} / 2+1\right)^{\frac{\gamma-1}{\gamma}}}-1}
$$

Applying this equation and rule of thumb to the RSW at all 4 span stations for the two unforced system cases yields the results in table 8 . The local Mach values indicate that the RSW at $2^{\circ}$ has a moderate strength shock, where shockinduced separation is unlikely. At $4^{\circ}$, the same analysis indicates a stronger shock, where shock-induced separation is likely. This also indicates that for the forced oscillation cases, where the angle of attack oscillates to a maximum angle of $3^{\circ}$, there is the possibility of separation and reattachment oscillations behind the shock.

Table 8. Local Mach number and shock-induced separation tendencies based on local pressure coefficient
\begin{tabular}{c||ccc||ccc} 
Wing & \multicolumn{5}{c}{$\alpha=2^{\circ}$} \\
Station & $C_{p}$, & $M_{\text {local }}$, & Separation & $C_{p}$, & $M_{\text {local }}$, & Separation \\
$\min$ & $\max$ & likely? & $\min$ & $\max$ & likely? \\
\hline 1 & -0.875 & 1.2094 & No & -1.078 & 1.3077 & Yes \\
2 & -0.873 & 1.2084 & No & -1.06 & 1.2987 & Yes \\
3 & -0.908 & 1.2249 & No & -1.108 & 1.3228 & Yes \\
4 & -0.928 & 1.2344 & No & -1.115 & 1.3263 & Yes
\end{tabular}

\section{V.B.3. Lower surface cusp region}

The comparison of the mean pressure coefficients for the unforced system showed consistent discrepancies between computational results and experimental data over the aft region on the lower surface. The measured distribution is flat instead of rounded like a classical supercritical distribution plot. One speculation is that these characteristics are being generated by an extreme thickening of the boundary layer; an alternate thought is that there is a local region of separated flow in this cusp area. In the case of the RSW at this particular test condition, the value of the pressure coefficient at the cusp leading edge is lower than what would usually be expected for the flow to separate, making the first speculation more likely. The frequency response functions that will be discussed subsequently show that the magnitude of the oscillatory response is near zero here. That is, the initiation of separation or the initiation of the boundary layer thickening is insensitive to angle of attack within the range of the tested oscillation magnitude. Examination of the lower surface mean pressure distribution in figure $17 \mathrm{~b}$ emphasizes this insensitivity to angle of attack, showing no variation over the lower surface from the $50 \%$ chord to the $65 \%$ chord location.

\section{V.C. Mean pressures calculated from oscillatory solution time histories}

The majority of the workshop analysts generated mean pressure distributions from steady-state solutions, but one analysis team provided mean results from both their steady-state solutions and their time-accurate forced oscillation solutions. All analysis teams provided a few time histories from their forced oscillation analyses, which will be examined in more detail later in this paper. From these time histories, mean values were calculated. The results of these mean calculations are shown in figure 18. In these plots, the lines show the static pressure distribution results submitted by the analysis teams. The symbols show the mean values calculated from the time histories submitted for the forced oscillation cases. The plots on the left hand side of the figure show the data for the entire chord station, while those on the right zoom in on the shock location.

An examination of all of the analysis results together (figures $18 \mathrm{a}$ and $\mathrm{b}$ ) shows that the mean values computed for the forward chord location agree very well, in general, with the submitted steady-state solution results. The points corresponding to locations aft of the shock onset for any particular analysis show substantial disagreement with the submitted mean values. This disparity is due to the nonlinearities introduced into the time histories due to shock oscillation. Unlike the experimental data, which contains shock motion for even the unforced system, the computational results of the steady-state solution are exactly that- steady.

Although not plotted separately, the time-history-calculated results from analysis team A correspond very closely to the submitted mean results. As will be seen in detailed examination of the time histories, these particular analyses did not predict any significant nonlinear behavior in the pressure time histories. Without the nonlinearities introduced by the shock motion, there is nothing to cause disparity between the mean values obtained using the two approaches. 
Focusing on the results from analysis team E (Appendix E and figures 18c and d), the points calculated from the time histories fall directly on the lines for some of their submitted mean data sets. These cases represent those where analysis team E performed the same analysis as above, calculating the mean from the forced oscillation data. The other data sets were generated from their steady-state analyses. Notice that the shock appears more vertical in the steady-state calculations. This is a symptom of the more aggressive shock motion that occurs in the forced oscillation case as compared to the unforced system case.

Results from analysis team $\mathrm{C}$ are also shown in separate plots (figures $18 \mathrm{e}$ and $\mathrm{f}$ ). The mean values computed from the forced oscillation time history data, shown by symbols, fall below the results calculated from the steady-state solution of the unforced system, shown by lines. This was also true for the results from analysis team F. Although in the earlier discussion of mean pressure distributions, teams $\mathrm{C}$ and $\mathrm{F}$ were grouped with teams $\mathrm{B}$ and $\mathrm{E}$, predicting a forward shock location, these two analysis sets (C and F) predict the shock just slightly ahead of the other two teams. Thus, their results at the forward chord location include shock oscillation effects. The nonlinear behavior observed in the time histories manifests as the mean value not matching the steady solution, even at this forward chord location.

\section{Frequency response functions}

Frequency response functions describe the periodic responses produced due to periodic input at discrete frequencies. Here, the responses consist of pressure coefficients and integrated load coefficients (i.e., lift coefficient, drag coefficient and pitching moment coefficient), and the reference or input refers to the angle of attack.

All FRF magnitudes shown in this paper have units of 1/deg; the coefficient forms of the responses are nondimensional and the angle of attack is in degrees. A separate section discussing the units is included later in this paper.

For the AePW, each analyst calculated the FRFs using their own methods. Fourier domain analysis was generally, although not universally, employed. The results were only requested at the frequency of forced oscillation or at the dominant response frequency determined by the analyst, defined as $f^{*}$. Parametric descriptions of the forced oscillation computations are given in tables 9 and 10 for the 10 and $20 \mathrm{~Hz}$ cases, respectively.

Table 9. Time-accurate computational parameters; $10 \mathrm{~Hz}$ forced oscillations

\begin{tabular}{|c|c|c|c|c|c|c|c|}
\hline & $\begin{array}{l}\text { Grid } \\
\text { Res }\end{array}$ & $\begin{array}{c}\text { \# of steps } \\
\text { per } \\
\text { cycle }\end{array}$ & $\begin{array}{c}\text { \# of } \\
\text { cycles } \\
\text { computed }\end{array}$ & $\begin{array}{c}\# \text { of } \\
\text { subiterations } \\
\text { per global } \\
\text { time step }\end{array}$ & $\begin{array}{l}\text { Time } \\
\text { record } \\
\text { length } \\
(\mathrm{sec})\end{array}$ & $\begin{array}{c}\text { Nyquist } \\
\text { frequency } \\
(\mathrm{Hz})\end{array}$ & $\begin{array}{l}\text { Fourier } \\
\text { frequency } \\
\text { resolution } \\
(\mathrm{Hz})\end{array}$ \\
\hline $\mathrm{A}$ & $M$ & 64 & 7 & variable & 0.7 & 320 & 1.43 \\
\hline \multirow[t]{3}{*}{ B } & $\mathrm{C}$ & {$[64,256]$} & 8 & 25 & 0.8 & {$[320,1280]$} & 1.25 \\
\hline & M & 64 & 8 & 25 & 0.8 & 320 & 1.25 \\
\hline & $\mathrm{F}$ & {$[64,1024]$} & {$[8,4]$} & 25 & {$[0.8,0.4]$} & {$[320,5120]$} & {$[1.25,2.5]$} \\
\hline $\mathrm{C}$ & M & {$[200,400,800]$} & {$[6,12,3]$} & 4 & {$[0.6,1.2,0.3]$} & {$[1000,2000,4000]$} & {$[1.67,0.84,3.34]$} \\
\hline \multirow[t]{3}{*}{$\mathrm{D}$} & $\mathrm{C}$ & {$[32,64,128]$} & 5 & {$[15,10,7]$} & 0.5 & {$[160,320,640]$} & 2.00 \\
\hline & M & {$[32,64,128]$} & 5 & {$[18,11,8]$} & 0.5 & {$[160,320,640]$} & 2.00 \\
\hline & $\mathrm{F}$ & 64 & 4 & 14 & 0.4 & 320 & 2.50 \\
\hline \multirow[t]{2}{*}{$E$} & $\mathrm{C}$ & 64 & 5 & 50 & 0.5 & 320 & 2.00 \\
\hline & M & 64 & 5 & 50 & 0.5 & 320 & 2.00 \\
\hline \multirow[t]{2}{*}{$\mathrm{F}$} & $\mathrm{C}$ & 64 & 6 & 50 & 0.6 & 320 & 1.67 \\
\hline & M & 64 & 6 & 50 & 0.6 & 320 & 1.67 \\
\hline
\end{tabular}

The reduced frequency, $k$, defined in equation 2 provides a non-dimensional relationship indicating the significance of the velocity introduced by the oscillation relative to the freestream velocity. In aeroelastic analyses, the reduced frequency is considered to be a critical parameter. The reduced frequencies for the RSW forced oscillation test cases are given in table 1 . They are sufficiently large that these cases can not be considered quasi-steady.

$$
k=\frac{\omega c}{2 V_{\infty}}
$$

Included in tables 9 and 10 are the Nyquist frequency and the Fourier frequency resolution. The Nyquist frequency 


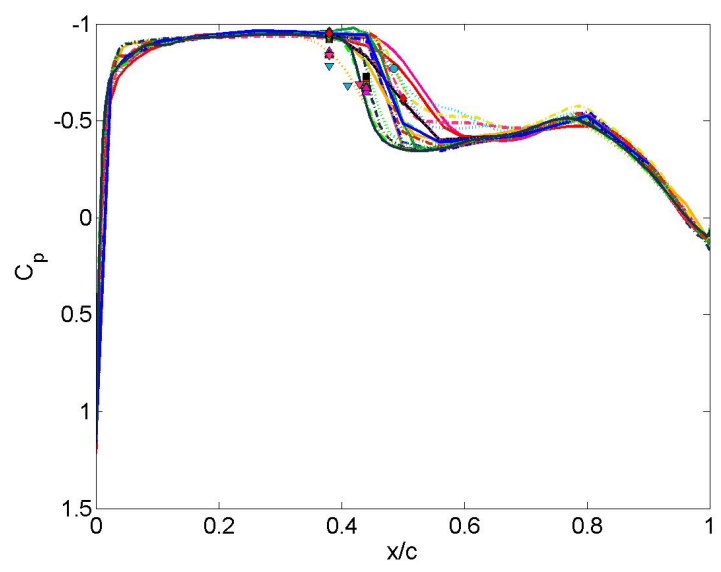

(a) All analysts, full chord shown.

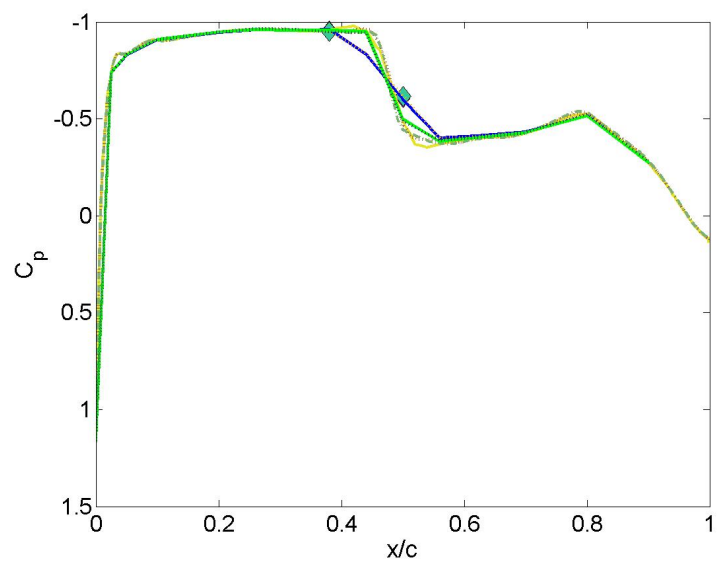

(c) Analysis team E, full chord.

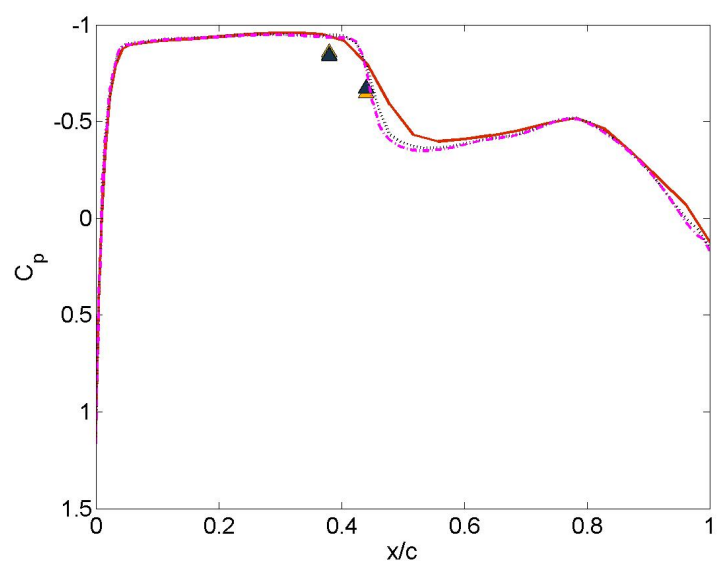

(e) Analysis team C, full chord.

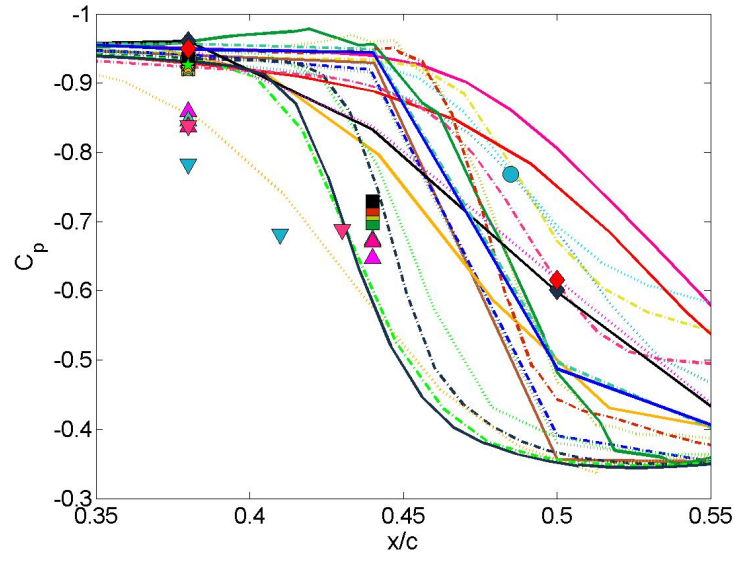

(b) All analysts, focused on shock region.

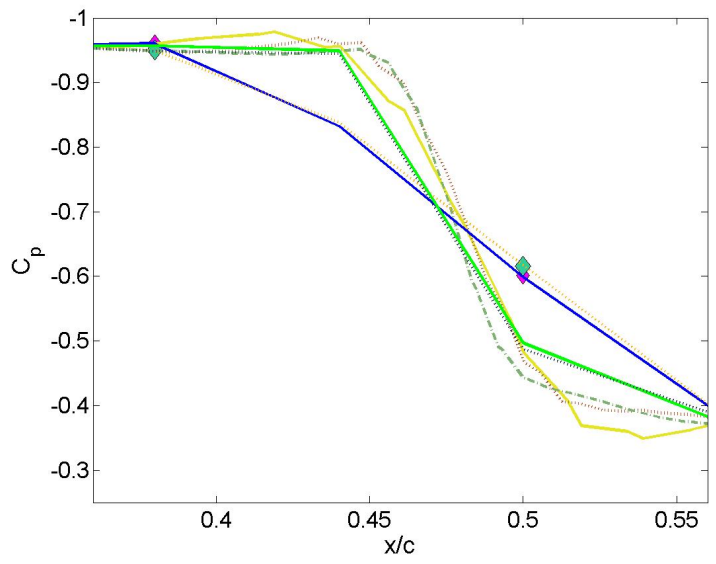

(d) Analysis team E, shock region.

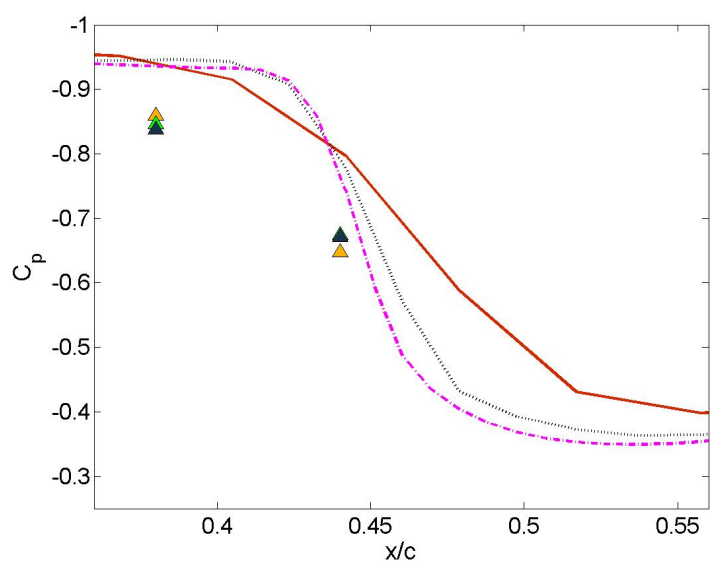

(f) Analysis team C, shock region.

Figure 18. Mean pressure distribution, computed from steady analyses and from time-accurate analyses; Upper surface; Station 2 ( $\eta=0.588)$. (Lines show analyst-submitted mean data; symbols show mean calculated from submitted forced oscillation time histories.) 
Table 10. Time-accurate computational parameters; $20 \mathrm{~Hz}$ forced oscillations

\begin{tabular}{|c|c|c|c|c|c|c|c|}
\hline & $\begin{array}{l}\text { Grid } \\
\text { Res }\end{array}$ & $\begin{array}{c}\text { \# of steps } \\
\text { per } \\
\text { cycle }\end{array}$ & $\begin{array}{c}\text { \# of } \\
\text { cycles } \\
\text { computed }\end{array}$ & $\begin{array}{c}\text { \# of } \\
\text { subiterations } \\
\text { per global } \\
\text { time step }\end{array}$ & $\begin{array}{l}\text { Time } \\
\text { record } \\
\text { length } \\
(\mathrm{sec})\end{array}$ & $\begin{array}{c}\text { Nyquist } \\
\text { frequency } \\
(\mathrm{Hz})\end{array}$ & $\begin{array}{c}\text { Fourier } \\
\text { frequency } \\
\text { resolution } \\
(\mathrm{Hz})\end{array}$ \\
\hline A & $\mathrm{M}$ & 64 & 6 & variable & 0.3 & 640 & 3,33 \\
\hline \multirow[t]{3}{*}{ B } & $\mathrm{C}$ & 128 & 8 & 25 & 0.4 & 640 & 2.50 \\
\hline & M & 128 & 5 & 25 & 0.25 & 1280 & 4.00 \\
\hline & $\mathrm{F}$ & {$[128,1024]$} & {$[5,2]$} & 25 & {$[0.25,0.1]$} & {$[1280,10240]$} & {$[4,10]$} \\
\hline $\mathrm{C}$ & M & {$[200,400,800]$} & {$[6,6,3]$} & 4 & {$[0.3,0.3,0.15]$} & {$[1000,2000,4000]$} & {$[3.35,3.35,6.7]$} \\
\hline \multirow[t]{2}{*}{$\mathrm{D}$} & $\mathrm{C}$ & {$[32,64,128]$} & 5 & {$[13,9,6]$} & 0.25 & {$[320,640,1280]$} & 4.00 \\
\hline & $\mathrm{M}$ & 64 & 5 & 9 & 0.25 & 640 & 4.00 \\
\hline \multirow[t]{2}{*}{$\mathrm{E}$} & $\mathrm{C}$ & 64 & 5 & 50 & 0.4 & 640 & 2.50 \\
\hline & M & 64 & 5 & 50 & 0.4 & 640 & 2.50 \\
\hline \multirow[t]{2}{*}{$\mathrm{F}$} & $\mathrm{C}$ & 64 & 6 & 50 & 0.3 & 640 & 3.33 \\
\hline & M & 64 & 6 & 50 & 0.3 & 640 & 3.33 \\
\hline
\end{tabular}

is important because it defines the maximum frequency of the flow phenomena that can be resolved. The Fourier frequency resolution is important because it defines the lowest non-zero frequency that can be resolved and determines the spacing of the frequencies available for defining frequency response characteristics.

The Nyquist frequency is determined by the sample length (time step size) measured in seconds, as shown in equation (3). It is determined by the requirement that each cycle must be represented by 2 or more time points in order to be discerned. Guidelines for data processing of experimental information advise the data analyst to sample the data at a rate 2 to 5 times faster than the Nyquist criteria would demand, relative to the highest frequency of concern for their application (i.e., desired bandwidth). The guideline of a factor of 5 has also been employed in analyzing CFD data. $^{22}$

$$
f_{\text {Nyquist }}(H z)=\frac{1(\text { cycle })}{2 \Delta t(\sec )}
$$

The Fourier frequency resolution is determined by the time record length on which Fourier analysis is performed, as shown in equation (4). Often, data records are not analyzed as a single record but are broken into overlapping segments for computing the Fourier series coefficients. The FRFs are computed using power spectral density and cross spectral density functions that are average values obtained for all of the data segments. ${ }^{27}$ This reduces the achievable frequency resolution but improves the confidence in the results. ${ }^{28}$ The frequency resolution values given in tables 9 and 10 correspond to the finest resolution achievable for a given data set- the case that uses the entire time record as a single analysis block.

$$
\Delta f(H z)=\frac{1(\text { cycle })}{T_{\text {ensemble }}(\mathrm{sec})}
$$

\section{VI.A. FRF results}

The FRFs for each of the two forced oscillation test cases, $10 \mathrm{~Hz}$ and $20 \mathrm{~Hz}$, are shown in Appendices C and D, respectively. For each case, there are sixteen plots: magnitude and phase for upper and lower surfaces at the 4 span stations. The results are coded in the same way as the mean pressure distributions- a different color for each analysis team and a different symbol for each data set submitted by a given analysis team. The magnitude of the frequency response function of $C_{p}$ due to $\alpha$ at a chosen frequency is plotted as a function of chord location in each of the plots identified as showing the magnitude. This relationship is expressed in equation (5).

$$
\left|\frac{C_{p}}{\alpha}\left(f^{*}\right)\right| v s \cdot \frac{x}{c}
$$


For the $10 \mathrm{~Hz}$ case, the computations predict a relatively consistent set of results on the lower surface and on the upper surface ahead of the oscillating shock. The large pressure pulses on the upper surface at the first 3 span stations represent the fluctuating shock strength and range of shock motion. There is significant variation among the computations in both strength and range. As we examine the data moving outboard towards the wingtip, the magnitude of the shock decreases until station 4 , where it is observed that for most of the computational results, the oscillations at the leading and trailing edges are of greater magnitude than the shock oscillation. For each span station, the aft-of-shock dynamic behavior has lower magnitude than the shock itself, but the distribution of the dynamics varies significantly among the computational results. Forward shock predictions tend to be coupled with overprediction of the dynamic response here, while aft shock predictions tend to be coupled with underpredictions.

To quantify this behavior, the data is analyzed to isolate the shock range of motion and strength. In the following discussion, the peak magnitude of the FRF is equated with the shock oscillation strength, and the range of shock motion is equated with the width of the dominant peak in the FRF, as exemplified in figure 14b. The peak width is defined using the points at the base of the peak, identified using an algorithm that examines the slope on either side of the maximum value. The algorithm was designed to find the point at which the slope either changes sign (as shown for the point identified aft of the shock in figure 14b), or the slope diminishes to below a prescribed value (as shown for the point identified ahead of the shock in figure 14b). Because the shock location plots contain so much data, a zoomed view of one analyst's data set is shown in figure 19 with a legend that applies to the plots in the left column of figure 20 .

The ranges of the computational results in figures 19 and $20 \mathrm{a}, \mathrm{c}, \mathrm{e}$, and $\mathrm{g}$ are shown by the colored plot features, while the center locations associated with several specific data sets are shown by the black plot features. One important result to note on the plots is the range of the shock oscillation for each of the forced oscillation cases. The range of the shock oscillation for the $10 \mathrm{~Hz}$ cases are shown by the blue-filled circles; the yellow-filled triangles show the shock oscillation ranges for the $20 \mathrm{~Hz}$ cases. This plot also contains the previously discussed mean data results for each computational case, shown by the solid horizontal lines. (Recall that the mean results represent the shock region of the unforced system pressure distribution, from the supersonic plateau ahead of the shock to the point at the foot of the shock or the aft end of the shock-induced separation region.) In the background of these computational data sets, the experimental data shock locations are represented. The vertical solid bar on each plot shows the shock center location obtained from the mean pressure distribution. The vertical dashed bar on each plot shows the experimental shock peak location for the 10 and $20 \mathrm{~Hz}$ cases, which are at the same chord station for all cases.

Examining the data first at span station $1, \eta=0.309$, the magnitude plots in Appendices C and D at both 10 and 20 $\mathrm{Hz}$ show the dominance of the oscillatory shock and also a large amplitude of the pressure field aft of the shock. The variations in the predictions of shock location and strength are shown in figure 20 a for this span station. They correlate well with the trends observed earlier in the mean pressure distributions. There are several interesting observations that can be made: 1) the shock oscillates over a wider range of the chord when forced oscillations are applied; 2) larger range of motion is observed for the $10 \mathrm{~Hz}$ forced oscillation than for the $20 \mathrm{~Hz}$ oscillation; and 3) the forced oscillation tends to move the range towards the leading edge. A subtlety of the results is that analysis teams B and E submitted their solutions at only the points corresponding to the experimental sensor locations, as requested by the OC. This is seen by comparing the vertical stacking of the shock motion range for analysis team B with the meandering stacks of analysis team $\mathrm{D}$.

The data is analyzed in the same manner for the other stations, figures $20 \mathrm{c}$,e, and f. Similar observations to those made above can be made at station 2, with the exception of a few results by analyst $\mathrm{D}$. At station 3, however, the data presents a more chaotic story. The station 3 plot shows several cases where the range of the dominant oscillation occurs at the leading edge. These are the cases where the shock oscillation has diminished such that the leading edge oscillation dominates the response. This is also the reason that several of analysis team D's results at station 2 are shown near the leading edge. At station 4, there are some cases where the dominant dynamic response is also shown at the trailing edge. These cases do not represent shock motion, as discussed previously.

Comparing across all span stations, the plots in the left-hand column of figure 20 show the agreement of the computational results and experimental data as the shock moves towards the leading edge. The range of the computational data on any given plot is an indication of the variation that the CFD solutions contain, taken as a whole.

The shock strength is shown in the right hand column of plots in figure 20. The plots show the consistent trend that the oscillatory magnitude is always less than the value that would occur if the shock completely oscillated across a sensor for each forced cycle. (This is one interpretation of the mean data shock strength.) Also consistently shown is that the $10 \mathrm{~Hz}$ oscillation magnitude is higher than the $20 \mathrm{~Hz}$ oscillation magnitude for every computational result, as well as the experimental data.

The plots show the consistency with which any single analysis predicted the shock strength. This is assessed by 


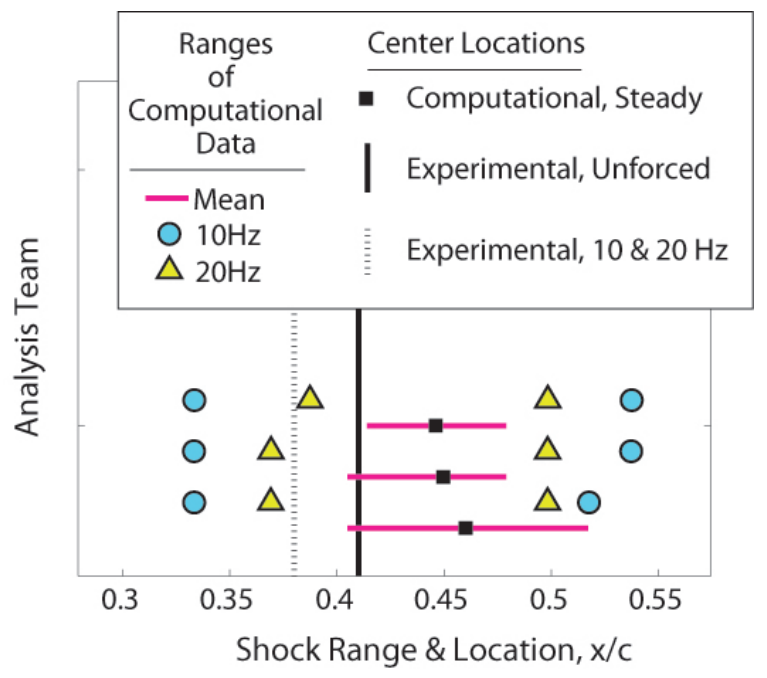

Figure 19. Location and range of upper surface shock oscillations, Station 2, Analyst C. (Experimental data ranges shown by shaded regions.)

examining the clustering of each team's results. The plots also show the consistency of the predictions among the analysis teams.

The lower surface magnitude plots in Appendices $\mathrm{C}$ and $\mathrm{D}$ show maximum response near the leading edge. The magnitude drops off quickly until the cusp region, where an aft plateau initiates. At the beginning of the cusp region, the magnitude plots are different at different span stations and for the two frequencies. At the inboard 2 span stations at $10 \mathrm{~Hz}$ the computational results show a flat-bottomed bucket in the magnitude. The experimental results show the magnitude going to 0 , at the inboard span station. Aft of the bucket, the computations appear to slightly underpredict the experimental dynamic response. At the outboard span stations, most analyses and the experimental data show an upward blip in the response at the cusp. At $20 \mathrm{~Hz}$, most of the computational results and the experimental data show a decided downward blip (decreased response) at the cusp.

With regard to the phase characteristics, the computational results are tightly bunched and follow the trends of the experimental data for the regions where this could be said of the magnitude. The region of disparity on the upper surface begins just ahead of the predicted shock location. Here, the computations have consistently lower phase angles than the experiment, starting at the leading edge of the predicted shock location. The regions of disagreement on the lower surface are in the cusp region and beyond, where the magnitude disparities were observed. The lower surface disparities are greatly reduced in comparison with those observed on the upper surface.

\section{VI.B. Interpreting the forced oscillation results, relative to the mean pressure distributions}

The mean values from the unforced system information lend insight into the expected results for the oscillatory solutions. Having a solution method and model capable of correctly capturing the unforced system pressure distribution is necessary for correctly predicting the oscillatory characteristics. Without a simulation that can correctly predict the shock location and strength of the unforced system, there is little hope of being able to correctly predict the geometric range of motion, strength or phase information associated with the oscillatory data. However, the forced oscillation solutions will introduce additional features that are not discernible from the unforced system data.

In the previous two section, the frequency response functions were defined mathematically and the results were described in terms of trends observed. The mean pressure distributions are revisited in this section to lend insight to their interpretation.

Moving the system means that the existing flow features, represented by the mean pressure distribution, will be under pressure to move or increase and decrease in magnitude. Features that are resistant or sluggish to respond to the forcing motion will exhibit phase loss relative to the forcing function.

The oscillations applied for the AePW had a magnitude of $1^{\circ}$, meaning that the airfoil was forced through sinusoidal motion between $1^{\circ}$ and $3^{\circ}$. There are no experimental data sets for these angles, but the distribution is likely bounded by the available data at $0^{\circ}$ and $4^{\circ}$. Figure 17 shows an example of the mean pressure coefficient experimental data for the $2^{\circ}$ nominal angle of attack at station 2 .

Quasi-steady analysis, near zero frequency, is useful for checking the feasibility of unsteady results and under- 


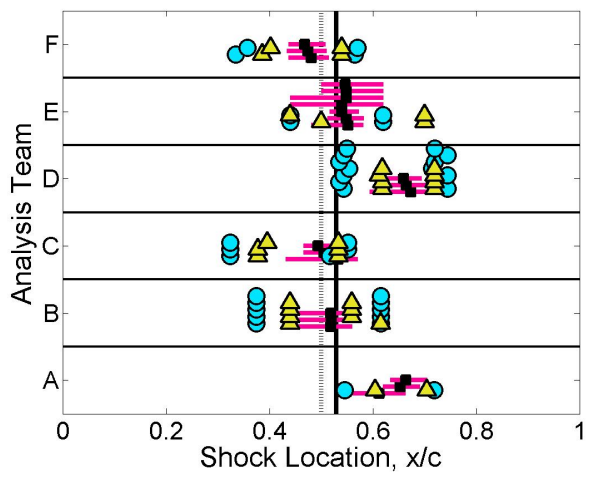

(a) Station 1, Location and Range.

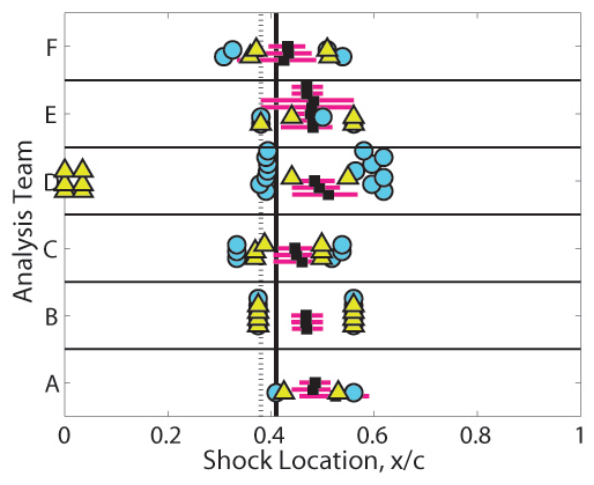

(c) Station 2, Location and Range.

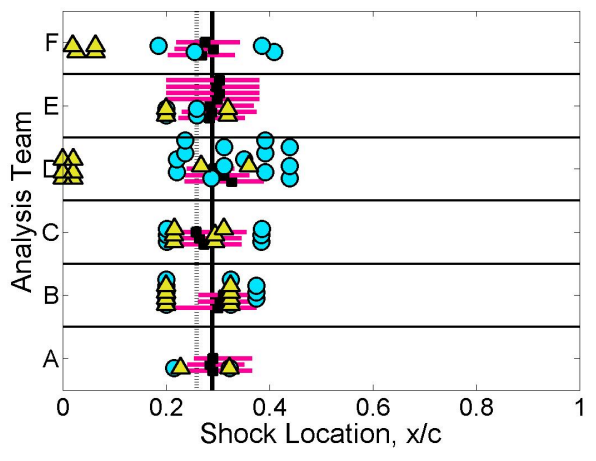

(e) Station 3, Location and Range.

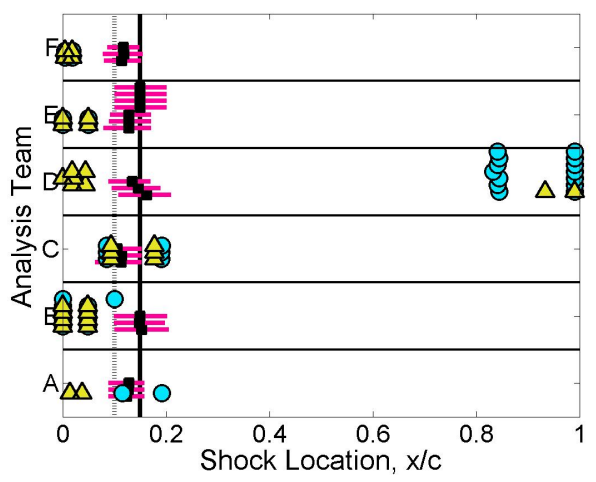

(g) Station 4, Location and Range.

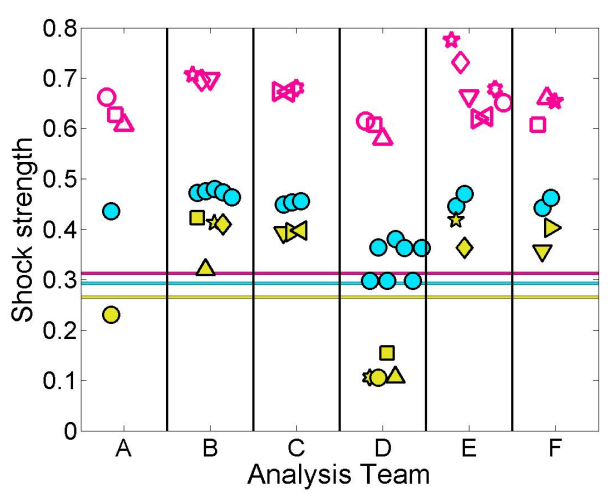

(b) Station 1, Strength.

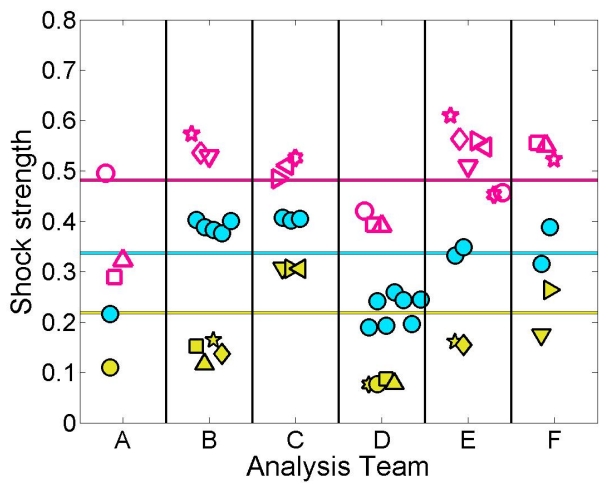

(d) Station 2, Strength.

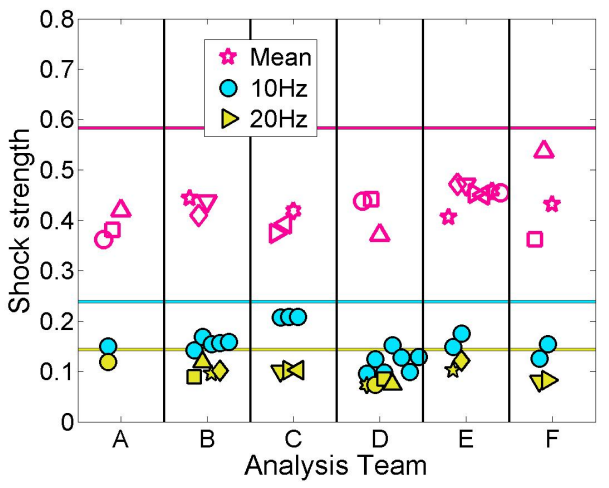

(f) Station 3, Strength.

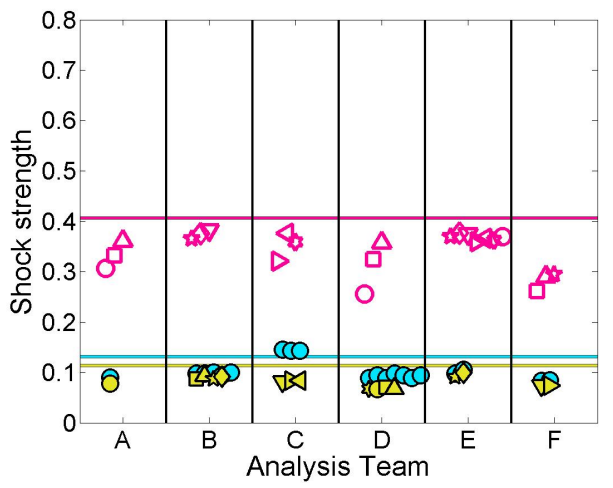

(h) Station 4, Strength.

Figure 20. Shock characteristics. 
standing some of the qualities of the frequency response functions. Quasi-steady cases can be classified as those cases where the phase of the FRF is $0^{\circ}$ or $180^{\circ}$, reflecting only the sign of the relationship between response and excitation. (Examination of the FRFs can also be performed using the real and imaginary components, rather than magnitude and phase. In examining real and imaginary components for a quasi-steady case, the imaginary component is zero.)

Forcing the airfoil to move in a quasi-steady manner (i.e., very slowly) from $0^{\circ}$ to $4^{\circ}$ would result in the pressure distribution moving from the $0^{\circ}$ line to the $4^{\circ}$ line. The FRF magnitude could be calculated by finding the difference between the two static lines at each point. The phase would be $0^{\circ}$ or $180^{\circ}$, as mentioned above, because the motion is so slow in our thought experiment that we have waited on all of the molecules. Also, moving this slowly, no oscillation-induced-velocity is created.

The upper surface shock is the dominant feature of the RSW test cases. Examining the information using the above process produces a result that incorporates both the shock location effect and the changing magnitude of the shock and pressure plateaus on both sides of the shock.

The previous discussion leads to the idea that the change in location of the shock as the system oscillates through different angles of attack will be a significant factor in the FRF. Using the information from the nominal angle of attack, the influence of the mean shock location movement can be isolated in a limited sense. The slopes of the mean pressure distributions have been calculated with respect to the chord location for the unforced system experimental data set, using a backward finite difference equation. These slopes are shown in figure 21. These results were calculated without information from the frequency response function calculations, yet they appear to capture an important feature of the FRFs, namely the oscillatory shock motion.

One discrepancy that should be noted occurs on the lower surface at the forward edge of the cusp. The mean pressure distribution in this region has a large slope, indicating that a large oscillatory response would be expected as the pressure distribution here moves forward and aft, following the forced motion. A quick check of the experimental FRFs in this region shows almost no response, i.e., the flow field in this region of the airfoil is insensitive to angle of attack changes. This is the same conclusion as that produced from examining the lower surface mean pressure distributions as a function of angle of attack, shown in figure $17 \mathrm{~b}$.

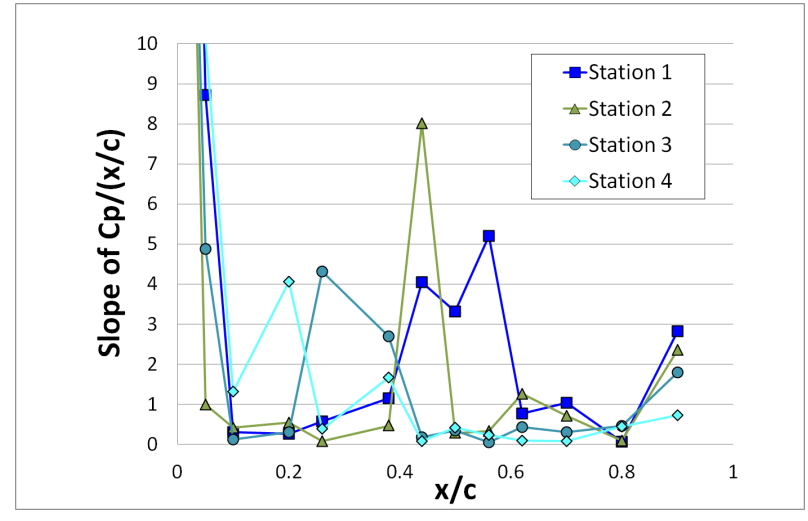

(a) Upper surface.

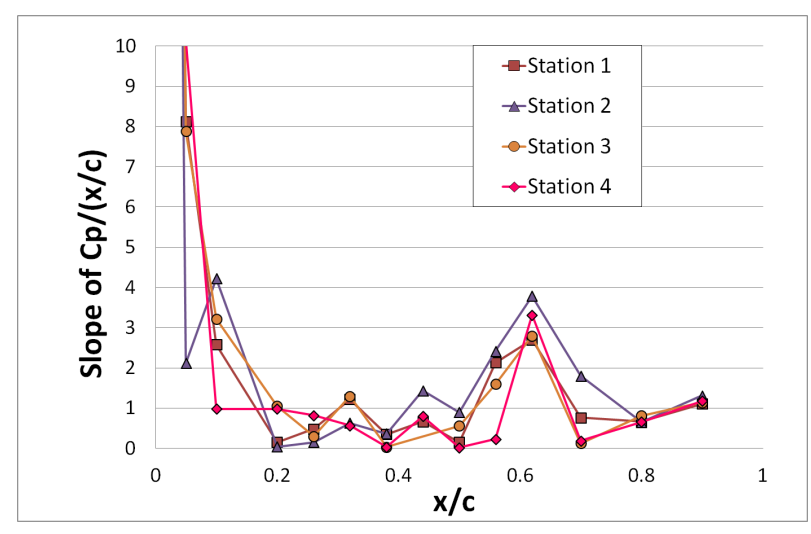

(b) Lower surface.

Figure 21. Experimental data; Slope of the mean pressure coefficient with respect to chord location; Unforced system data.

\section{Time histories from the computational results}

A few geometric locations were selected for examining the time history responses from the computational models. Each analyst was asked to submit data for station $2, \eta=0.588$, at two locations:(1) at a given location, $\mathrm{x} / \mathrm{c}=0.38$, and (2) at the location of maximum FRF response. This second location was specified to capture the behavior of the oscillating shock. The time histories from the submitted medium grid solutions for the $10 \mathrm{~Hz}$ oscillations are shown in figure 22. In order to compare the dynamics present, the time histories were synchronized based on the minimum value (peak on the plot) and time-adjusted to 0 .

The common location for submitting time histories, $\mathrm{x} / \mathrm{c}=0.38$, was chosen in an attempt to find a location that would be at a high magnitude of the mean pressure coefficient, without including shock dynamics. From the time histories shown, figure 22a, it can be discerned that for analysis teams A, D and E, this was the case. These time histories show near-perfect sinusoidal pressure responses. The results from analysis team B indicate that this chord 


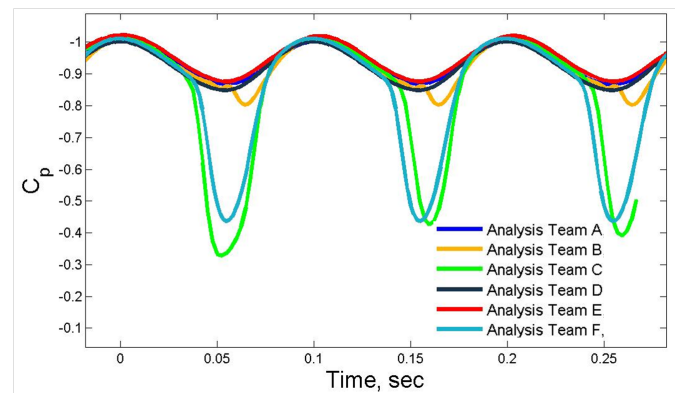

(a) Responses at forward chord location, $\mathrm{x} / \mathrm{c}=0.38$.

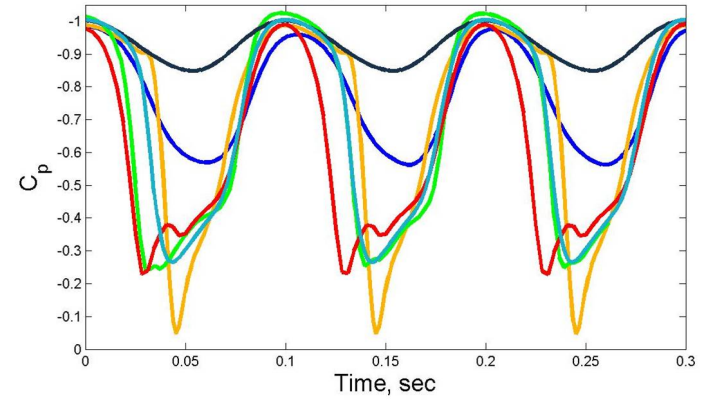

(b) Responses at chord location of maximum FRF resposne.

Figure 22. Time histories of pressure coefficient; $10 \mathrm{~Hz}$ forced oscillation computational results; Upper surface; Span station 2.

location is very near the forward limit of the oscillation range of the shock being predicted by their computations. This is indicated by the relatively small nonlinear portions of the otherwise sinusoidal pressure response. The time histories from analysis teams $\mathrm{C}$ and $\mathrm{F}$ show that their results predict the shock to oscillate further forward than analysis team B. If they were predicting that the shock oscillation range was entirely forward of this location, you would anticipate a flattening of the response at the bottom of the time history, indicating that the pressure plateau aft of the unforced system shock had now moved past this location.

The second time history location is different for each analyst. Their choice in chord location was based on where their shock oscillations had the most significant influence on the pressure coefficient. The synchronized time histories for these chord locations are shown in figure $22 \mathrm{~b}$. The results from analysis team A appear fairly sinusoidal, emphasizing the compatibility of the mean value calculations discussed previously for analysis team A. Analysis team D's results are also sinusoidal, but reflect their choice of chord location-it was significantly forward of their shock location. The other four time histories for teams B, C, E and F show significant nonlinear behavior as the shock oscillates forward and aft of the selected chord location.

\section{Influences on results}

\section{VIII.A. Influences of analysts' choices}

The choices made by the analysts in setting up their computational models, tables 4 and 5 , influence the outcome of the solutions. One objective of the AePW was to identify the significant factors. Unfortunately, all attempts to discern this information from the RSW data set have been unsuccessful. Contributing factors to this lack of success are the overall small number of analyses that were performed, the small number of analyses that were performed for any given parameter choice, the lack of diversity (in the case of the turbulence model), and the vast array of differences among the methodologies employed.

Based on the lessons learned from prior workshops, DPW and HiLiftPW, some variables that were examined included: turbulence model, solver type (cell- or node-based) and grid resolution. Example results are shown in figure 23 , sorting the data by each of the above variables, but they offer little in the way of conclusions. Even when sorting by grid factor, the dominant differentiator appears to be "analyst." (In the figures, the different options for the parameter being sorted are shown using different colors.)

Three factors that may have had a significant influence on the results are placement of the wing relative to the wind tunnel wall boundary layer, wing tip modeling and solution methodology. The significance of these effects cannot be statistically isolated from the effect of being submitted by different analysis teams because each case either has one analysis team that utilized a different option (wing tip modeling or wing placement relative to the boundary layer) or every analysis team utilized a different option (solution methodology). Plots sorting by the wing tip model are shown in figure 24. The information in table 5 shows that this will isolate analyst C. Plots sorting by location of the wing relative to the wind tunnel wall, that is, isolating analyst $\mathrm{D}$, are shown in figure 25 . It should be pointed out that analysis team D is also the only team that used the SST turbulence model instead of an SA model. From the figures, it is observed that the first modeling difference indicates more evident changes near the wing tip, while the second indicates more evident changes near the wing root. 


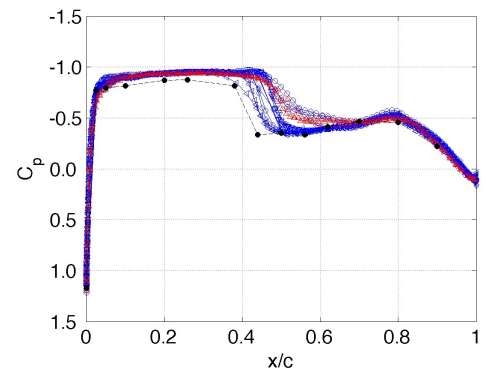

(a) Turbulence model (Blue:SA; Red:SST).

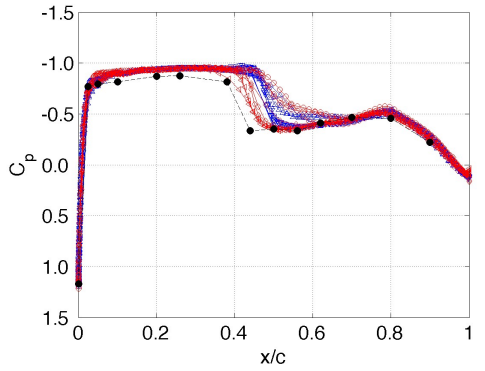

(b) Solver type (Blue: Node-based; Red: Cellbased).

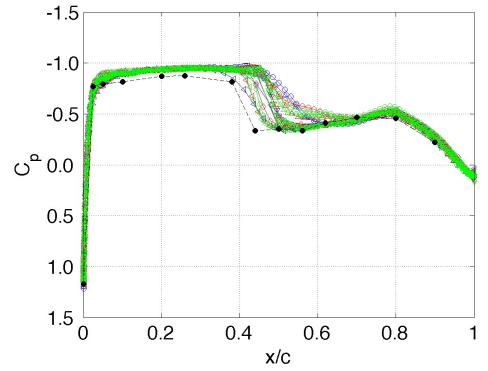

(c) Grid Resolution (Blue: Coarse; Red: Medium; Green: Fine).

Figure 23. Unforced system mean pressure coefficients, sorted by identified variables,(Case: $\alpha 2^{\circ}$, Upper surface, Station 2$)$.

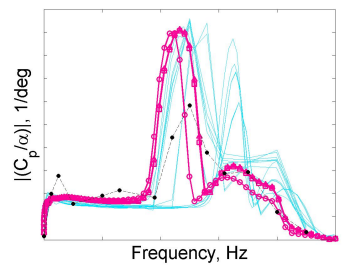

(a) Station 1.

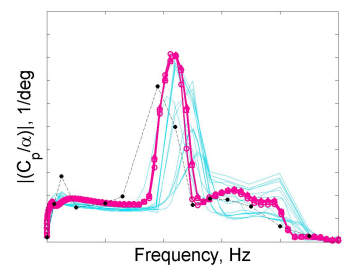

(b) Station 2.

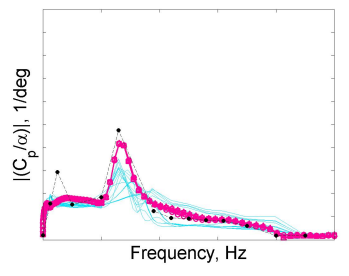

(c) Station 3 .

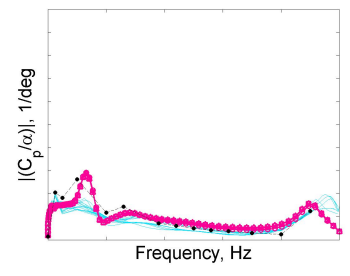

(d) Station 4 .

Figure 24. Sorting results based on wing tip modeling (Red: scarfed tip; Blue: tip of revolution); Magnitude of FRFs at $10 \mathrm{~Hz}$.

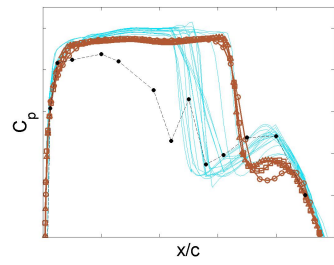

(a) Station 1 .

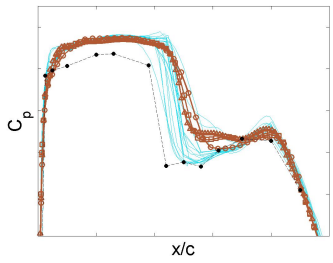

(b) Station 2 .

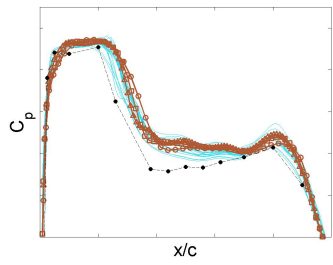

(c) Station 3 .

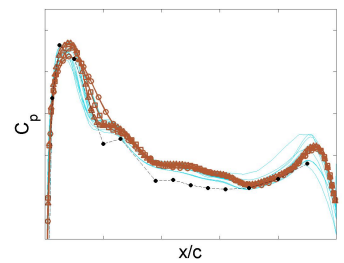

(d) Station 4 .

Figure 25. Sorting results based on wing span (Red: 48”, Blue: 55”); Mean pressure coefficients of the unforced system at $2^{\circ}$. 


\section{VIII.B. Influences of transition strips}

For all AePW RSW experimental data points, transition strips were located on the upper and lower surfaces of the model at the $6 \%$ chord, attempting to fix the location of laminar to turbulent transition. The potential influences of the transition strips on the flow field in three regions of the wing are discussed below: the upper surface leading edge region, the upper surface shock region and the lower surface cusp region.

The upper surface leading edge region is discussed because the experimental FRFs that have been presented show a significant peak in the magnitude of the response at each of the span stations for the sensor located at $\mathrm{x} / \mathrm{c}=0.05$. This region is one area of mismatch between the computational and experimental FRFs. The proximity of this peak to the transition strip location brings up the question as to whether or not the peak is a local response due to the strip, producing this mismatch with the computational results. All of the computational results were performed under the assumption that the flow was fully turbulent; no one analyzed the RSW to include transition effects. A few computations (analysis teams B and E) do indicate this behavior for the outboard span station, but these are the exceptions. Otherwise, the computational results appear to miss this behavior.

There are three available experimental FRF data sets with transition strips: the two AePW test cases at 10 and 20 $\mathrm{Hz}$ and an additional case at $15 \mathrm{~Hz}$. The FRFs for each of these three cases were compared to data sets obtained at equivalent test conditions without transition strips. An example plot at the second span station is shown in figure 26 for AePW test case 3 ( $10 \mathrm{~Hz}$ excitation) and its companion data set without transition strips. The percent differences between the magnitudes on the upper surface at $\mathrm{x} / \mathrm{c}=0.05$ were computed for each of the three companion data sets $(10,15$ and $20 \mathrm{~Hz})$ at the 4 span stations. For each span station, the mean value of the percent differences is shown in table 11. The data with transition strips always has a higher value than without transition strips. The largest differences are at the inboard span station, while the differences at the outboard span station are considerably smaller. As indicated by the data in the first data column of table 11, the transition strips had only a 2-15\% effect on the local pressure coefficient fluctuation.

The AePW experimental data set (i.e., with transition strips) was compared with the computational data in a similar manner. The computational data was only available for the AePW test cases with frequencies of $10 \mathrm{and} 20 \mathrm{~Hz}$. At each span station, the computational results at the 5\% chord locations were averaged, and the percent difference with respect to the experimental data was computed. All data at 10 and $20 \mathrm{~Hz}$ was combined to compute the mean percent difference, shown in the second data column of table 11 . The data shows that there is a $23-55 \%$ difference at this chord station. This is a substantially larger difference than that observed due to the presence of the transition strips in the experimental data set.

To calculate lower bounds on the percent differences, the maximum values from among all of the computational data sets at the $5 \%$ chord locations were used in these calculations. These results, shown in the last column of table 11, predictably show smaller differences than produced by the mean value. However, even this representation leads to the conclusion that these differences are substantially larger than those that could be attributed to transition strip influences alone. Thus, it is concluded that the presence of the transition strips accounts for some of the leading edge oscillations, but there is a substantial portion of the disagreement with the computational results that is unrelated.

Table 11. Influence of transition strips for all frequency cases combined; Upper surface at $\mathrm{x} / \mathrm{c}=\mathbf{0 . 0 5}$

\begin{tabular}{c|c|c|c}
\hline & \multicolumn{3}{|c}{ \% Difference } \\
& \multicolumn{3}{|c}{ Experiment with transition strips compared with: } \\
\hline & $\begin{array}{c}\text { Experiment without } \\
\text { transition strips }\end{array}$ & $\begin{array}{c}\text { Computations, } \\
\text { Mean value }\end{array}$ & $\begin{array}{c}\text { Computations, } \\
\text { Maximum value }\end{array}$ \\
\hline \hline 1 & 15.3 & 39.3 & 28.8 \\
2 & 7.2 & 41.6 & 23.2 \\
3 & 9.2 & 55.5 & 39.1 \\
4 & 2.2 & 23.4 & 8.6 \\
\hline \hline
\end{tabular}

Referring again to figure 26a, several other features are prominent in terms of the influence of the transition strips on the frequency response function. The oscillatory shock is observed to be located further forward for the case with the transition strip. Although the magnitude of the shock oscillation is shown to be larger for the case without the transition strip in this particular data set, this is not a consistent feature found in the data. The pressure sensors are 


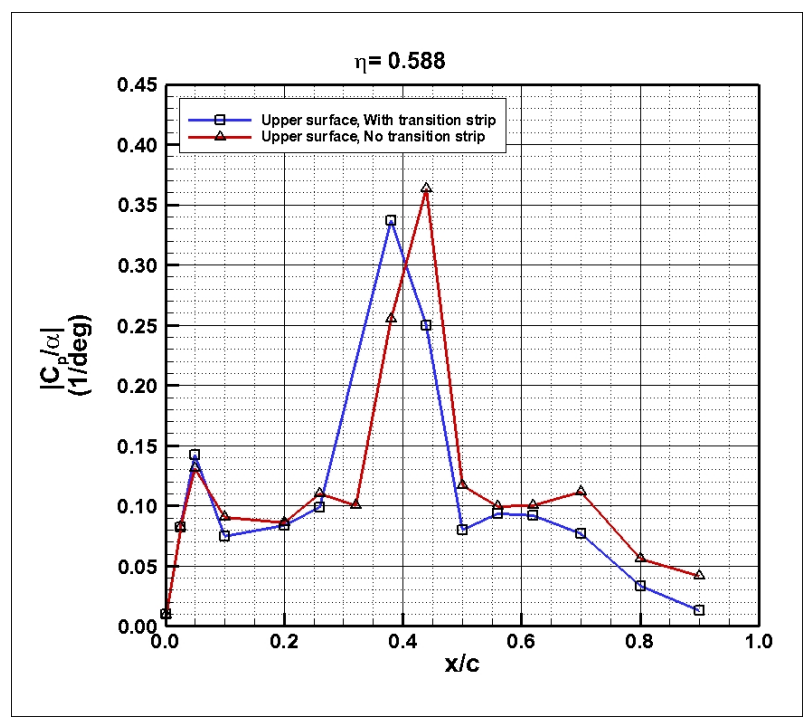

(a) Upper surface.

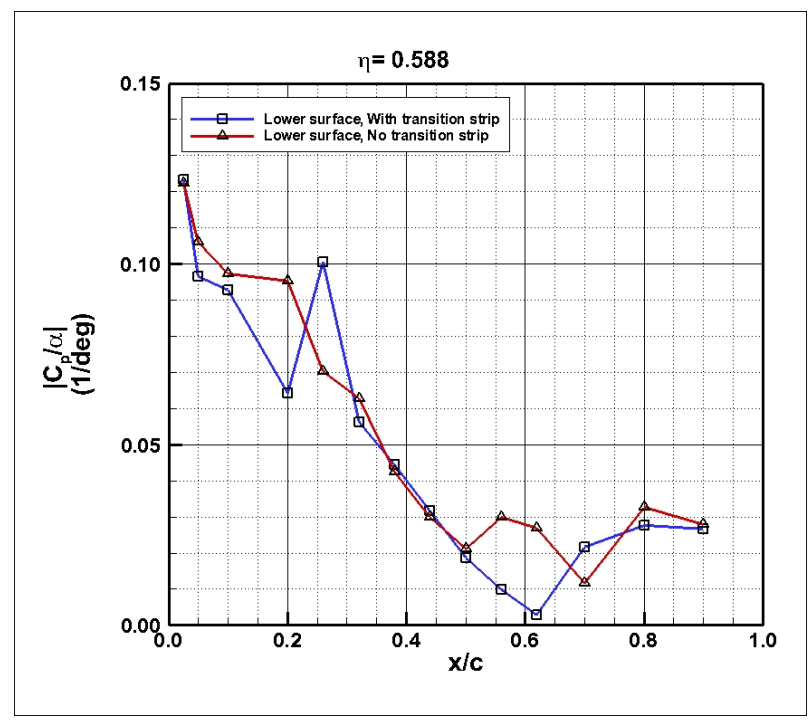

(b) Lower surface.

Figure 26. Experimental data with and without transition strips; Station 2; Magnitude of the FRF at $10 \mathrm{~Hz} ; \alpha=2^{\circ}$.

spaced too far apart to be able to comment with any assurance as to which case produces a higher amplitude in the oscillating shock response. Aft of the shock, the shape of the pressure recovery region changes when the transition strips are not present.

The computational data for this example is shown with both corresponding experimental data sets in figure 27. Figure 27a shows that the computational FRFs on the upper surface are consistently more in line with the transitionstrip-free experimental FRFs. Comparisons between the experimental and computational FRFs also show disagreement on the lower surface in the region of the cusp, as shown in figure 27b. It is interesting that there is a similar disagreement between the two experimental data sets with and without the transition strips. As discussed previously, the FRF is related to the unforced system (mean) pressure distribution. Comparison of the unforced system mean distribution shows different characteristics with and without transition strips over the aft portion of the lower surface. With transition strips, the pressure distribution is flatter than the distribution of the case without transition strips. It is unclear whether this behavior is specifically related to the transition strip presence or if it is just a data repeatability effect.

The closer match between the computational data and the experimental data without transition strips is likely fortuitous. It is also possible that the transition strips used in the experiment were not appropriately sized. The RSW data was originally generated for transonic unsteady aerodynamic code development. At the time of the data acquisition, the majority of unsteady aerodynamic codes in development included inviscid flow assumptions and viscous fluid properties were not included in the aerodynamic computations. Those involved in the original RSW program chose to perform the majority of their testing without transition strips, and compared their computational results only to the data without transition strips present. ${ }^{10}$ It is unclear whether they had insight into the quality of the data sets with and without transition strips, or if they were simply using the standard practice of the day. The transonic shock location can be a strong function of the state of the incoming boundary layer ahead of the shock and the AePW computations generally predict a shock location aft of the experiment, indicating that they may be predicting a thinner boundary layer ahead of the shock. Whether a case of an overly thin boundary layer predicted in the computations, or an overly thick boundary layer due to the transition approach employed in the experiment, or a combination of both, the boundary layer produced in the experiment without transition strips appears to be better captured in the AePW computations than the boundary layer produced by the experiment with transition strips present.

\section{VIII.C. Postprocessing}

The processing of computational data is very different from the processing of experimental data. Experimental data sets for dynamic cases are generally of sufficient time length to perform data averaging. ${ }^{28}$ Experimental data sets are also noisy, containing wide-spectrum sources of noise, including airstream turbulence. Computational data usually has a very short time record, but it is relatively noise-free. The computational data also offers the advantage that the user 


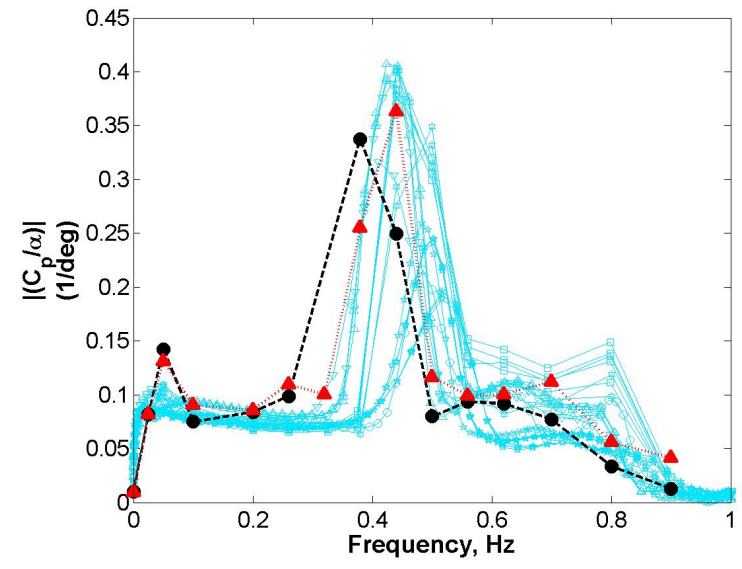

(a) Upper surface.

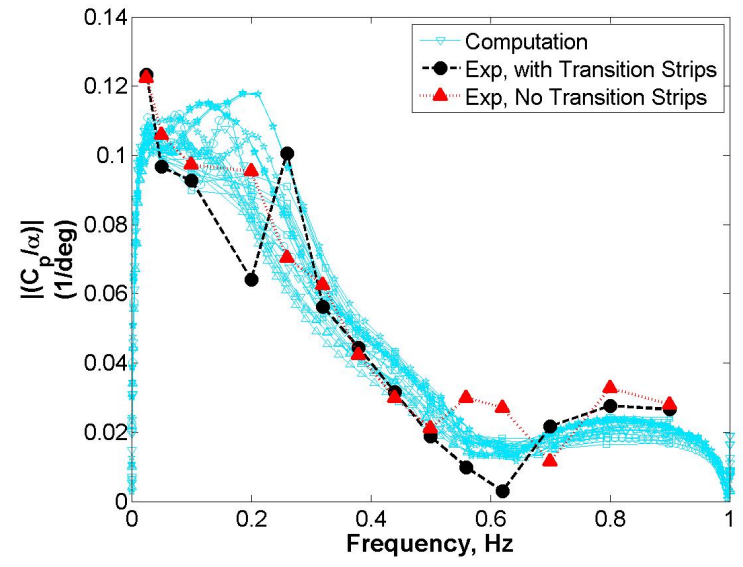

(b) Lower surface.

Figure 27. Effect of transition strips; Comparison with computational data; Station 2; Magnitude of the FRF at $10 \mathrm{~Hz}$.

specifies how the data samples will be spaced in time, relative to the oscillation being performed. This has been done for at least one experimental data set as well (HIRENASD) and has been attempted for some system identification methods, but real-world implementation issues can easily derail the benefits. It has been demonstrated that even small errors in implementation reintroduce the requirement for data averaging. ${ }^{29}$

In performing the postprocessing of the computational data, the Fourier transform and the frequency selection process eliminate all information except that at the assumed frequency. Fourier analysis at an inaccurate frequency leads to leakage and generally results in a reduced response being represented. Presence of nonlinear effects which seem obvious in the time histories are ignored in our current methods, even though their presence in the system can contaminate the computed response at the selected frequency.

The total record length determines the frequency spacing in a Fourier analysis. If the frequency content is exactly at one of the Fourier frequencies and the data is analyzed as a single window containing an integer number of cycles, there are no leakage errors introduced. ${ }^{29,30}$ These are the assumptions that were made in much of the postprocessing of the computational data for this workshop. A few analysis teams used the periodogram method in constructing their frequency response function estimates. In these cases, the record length is also important because it limits the number of windows of data that can be obtained for a given level of overlap. For the computational data, the information at most chord locations was fairly periodic and rectangular windowing with a large amount of overlap (between 75\% and $95 \%$ ) was used to obtain a larger number of data windows. One analysis team, C, performed least squares fits of their pressure time history data to a sinusoidal function of preassigned frequency. While their methodology appears fundamentally equivalent to performing a Fourier anlaysis, they also performed manual inspection of the results for single windows of data containing integer numbers of cycles. They added cycles of computational results to their data window until they were satisfied with their results. Analysis team B used similar logic in determining the data processing window size used in their Fourier analysis, although convergence was based on minimizing the standard deviation of the periodograms used to compute their Fourier coefficients.

The time step size is also an important factor in representing the data. The time step size determines the Nyquist frequency, which sets the upper bound on the frequency of flow phenomena that can be resolved. For this study, the Nyquist frequency (and advisable frequency, between 1/5 and 1/2 of the Nyquist) is more than satisfied by the time step sizes chosen.

\section{Statistical analysis}

Statistical analyses of the RSW data sets were performed using methods identical to those previously employed in the DPW ${ }^{31}$ and HiLiftPW. ${ }^{32}$ Methods for assessing outlier data sets and tracking change in variation level were employed. In the prior workshops, the first moment of the distribution was chosen to be represented by the median value, rather than the mean in order to make the statistic robust to outliers.

Scatter bounds were calculated to identify outlier data sets. This was accomplished using equation 6 . sIn equation $6, \hat{\mu}$ is the computed estimate of the median value, $\hat{\sigma}$ is the computed value of the standard deviation and $\mathrm{K}$ is 
the confidence interval coverage factor. Identifying a data set as an outlier means that some aspect of the solution was significantly different from the others and its causes should be investigated. The selection of a larger confidence interval coverage factor will result in fewer data sets being identified as outliers. For the previous workshops, a coverage factor of 3 or the $\sqrt{3}$ was used. For AeWPW, we utilized the latter choice. Even with the statistical method, there is a great deal of subjectivity, first in the choice of the coverage factor and second in the choice of importance given to different output parameters. The decision to define a data set as an outlier was made based on 2 out of 3 unforced system integrated loads being statistically an outlier.

$$
\hat{\mu} \pm K \hat{\sigma}
$$

The coefficient of variation, calculated using equation 7 , was used to compare the variation among the submitted computational data sets. Plots showing the details of the analysis for the unforced system at $2^{\circ}$ are shown for the lift coefficient (figure 28a), the drag coefficient (figure 28b) and the pitching moment coefficient (figure 28c). In each of these cases, the scatter range and coefficient of variation are shown for the analysis of the data set as a whole, rather than by grid resolution.

$$
C_{v}=\hat{\sigma} / \hat{\mu}
$$

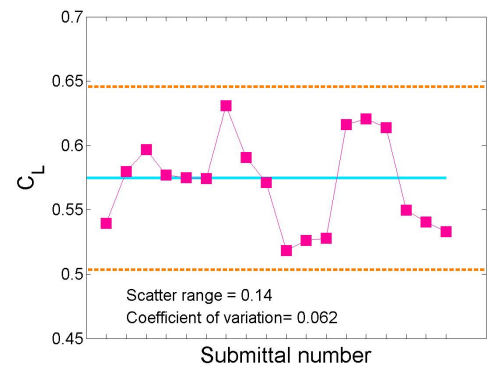

(a) Lift coefficient.

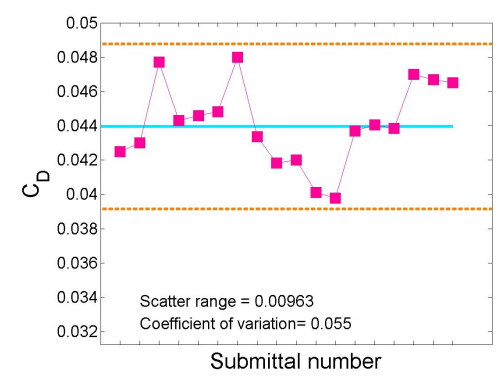

(b) Drag coefficient.

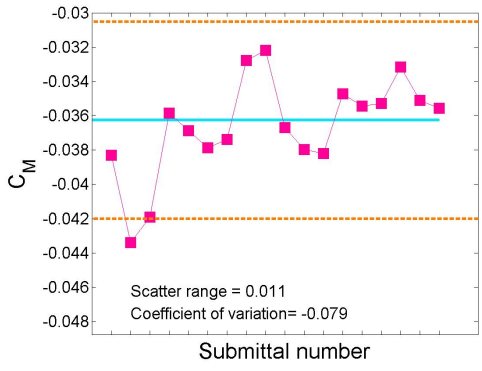

(c) Pitching moment coefficient.

Figure 28. Assessment of statistics of computational results; Unforced system integrated coefficient; Case $1 \alpha=2^{\circ}$.

These statistics can be useful in tracking improvements in varability among computations and in identifying outlier data sets. The statistical assessment is of use because it gives a quantification, although still subject to subjectivity, of code-to-code variation. In the case of the RSW data sets, the outlier points are obvious, without any need to perform the statistical calculations.

There are several points that should be made regarding the results presented here. First, the number of data sets used to compute the statistical quantities for each of the grid resolutions is no greater than six. Fewer data sets means that we have lower confidence that the scatter bounds accurately capture the population. Second, there are known important differences among the analyses. The convergence criteria discussion covered many of the suspected contributors towards the variations indicated here- namely wing span, integration area and reference length. The wind tunnel wall boundary layer, discussed throughout this paper, is another known significant source of variation in these results. Additionally, each analyst used a different set of grids and made different flow solver choices. The comparatively large variations described by the statistical results are likely reflections of these known variations in these choices. Third, the statistics shown represent only scatter in the between-computation comparisons. They do not reflect how well or how poorly the computations predict the physics of the problem.

\section{Comments on experimental data sets}

\section{X.A. Sensor health assessment}

In previous publications documenting testing of the $\mathrm{RSW},{ }^{10-13}$ it was noted that the lower surface pressure sensor at station $3, \eta=0.809$, at $\mathrm{x} / \mathrm{c}=0.45$ malfunctioned during the entire test. Over the course of the AePW-related RSW data set examination, several additional sensors were determined to have been faulty during the wind tunnel test. They have all been removed from the results shown in this paper.

The majority of the faulty sensors were on the upper surface at $\mathrm{x} / \mathrm{c}=0.32$, for all span stations. The RSW testing was conducted by first acquiring a full data set without transition strips on the model. Near the end of the test, the 
model was modified by adding the transition strips, and a reduced set of data was acquired. Direct comparison of the data with and without transition strips has shown that the responses of these upper surface sensors at $\mathrm{x} / \mathrm{c}=0.32$ were greatly reduced after the model modification. Changes to the aerodynamic characteristics were eliminated as the source of this response reduction by comparisons made among the different data sets without transition strips. These sensors were still partially functional, as the phase results that were examined still tracked with the rest of the airfoil.

The other sensor that appeared to behave in a questionable manner is located on the upper surface at the inboard span station at $\mathrm{x} / \mathrm{c}=0.44$. Detailed comparisons of the data sets with and without transition strips and with changing Mach number indicate that this sensor is not, in general, bad. A divot observed in the pressure distribution for the AePW test condition at this location is very near the rotation axis, $\mathrm{x} / \mathrm{c}=0.46$. There are many small geometric features of the splitter plate and turntable hardware that could be responsible for producing this divot. The divot has also been shown to move with Mach number, indicating that it is more likely a flow field feature, rather than a sensor issue. Interestingly, other references (e.g. Harris ${ }^{16}$ ) show test data from supercritical airfoils show a similar divot, but the authors do not provide comment.

\section{X.B. Notes regarding units}

The RSW data sets have been widely distributed in reduced-data form. The FRFs are computed as the non-dimensional pressure coefficients due to pitch angle oscillations. A source of confusion in using the RSW data set was in interpreting the units of the published frequency response functions.

Having culled through all of the relevant publications and sorted out this issue, we document it here for posterity. The RSW data was originally published in $1983^{10}$ and $1984^{10}$ by Ricketts et containing plots and tables of FRF results that are in 1/deg. A journal article was published in 1984 by Ricketts et al. ${ }^{10}$ containing plots in units of $1 /$ deg (Figures $5,6,9,10,13$ and 14). The plots in the journal article are correct; the currently available pdf version of the article (as of November 2012) has vertical axes which have lost the decimal points, making them appear 10 times larger in general than the proper values. The RSW data set was revived in 1999 by Bennett and Walker, ${ }^{13}$ where they converted the data to $1 / \mathrm{rad}$, although no units for the FRFs are specified in the document. This holds true for the subsequent documents that were produced: an AGARD report ${ }^{33}$ and an RTO report. ${ }^{34}$ It is this last report that has an accompanying set of experimental data that served as the basis for the AePW experimental database.

The FRFs published at the AePW in April 2012 are in units of 1/rad. To have a set of consistent units between the RSW and BSCW configurations, it was decided to change the units to 1/deg for AePW publications. All of the FRFs shown in this paper are in $1 / \mathrm{deg}$.

\section{Concluding Remarks}

The Rectangular Supercritical Wing proved to be a challenging configuration to analyze, due in large part to the mounting of the wind tunnel model within the wind tunnel boundary layer. The computational methods applied to examine this configuration were varied, resulting in comparison data sets that could not be dissected to determine causal relationships. The configuration was instructive in terms of investigating flowfield behavior for the mean pressure distribution and for frequency response functions. Many characteristics of the data sets were not fully explored due to lack of time history information from the experimental data set. The observations made with regard to the RSW and its associated data will serve as a basis for proceeding with future analyses using the other two configurations from the Aeroelastic Prediction Workshop, the BSCW and the HIRENASD model.

\section{Acknowledgments}

The authors gratefully acknowledge the AePW team, particularly the RSW analysts and the AePW Organizing Committee for their guidance and discussions.

\section{References}

\footnotetext{
${ }^{1}$ Levy, D. W. et al., "Summary of Data from the First AIAA CFD Drag Prediction Workshop," AIAA Paper 2002-0841, Jan. 2002.

${ }^{2} \mathrm{http} / / /$ aaac.larc.nasa.gov/tsab/cfdlarc/aiaa-dpw/, August 2010.

${ }^{3}$ Rumsey, C. L. et al., "Summary of the First AIAA CFD High Lift Prediction Workshop," AIAA Paper 2011-0939, Jan. 2011.

${ }^{4}$ http://hiliftpw.larc.nasa.gov/, August 2010.
} 

2013.

${ }^{5}$ Heeg, J. et al., "Overview of the Aeroelastic Prediction Workshop," Tech. rep., 51st AIAA Aerospace Sciences Meeting, Grapevine, TX, Jan

${ }^{6}$ Schuster, D. et al., "Analysis of test case computations and experiments from the first aeroelastic prediction workshop," AIAA paper, 51st AIAA Aerospace Sciences Meeting, Grapevine, TX, Jan 2013.

7"http://www . aeronautics .nasa.gov/atp/facilities/tdt/index.html".

${ }^{8}$ Cole, S. R. et al., "Test Activities in the Langley Transonic Dynamics Tunnel and a Summary of Recent Facility Improvements," AIAA Paper 2001-1958, April 2001.

${ }^{9}$ Piatak, D. and Cleckner, C., "Oscillating Turntable for the measurement of unsteady aerodynamic phenomenon," Journal of Aircraft, Vol 14, No. 1, Jan-Feb 2003

${ }^{10}$ Ricketts, R. H., Sandford, M., Seidel, D., and Watson, J., "Transonic pressure distributions on a rectangular supercritical wing oscillating in pitch," Journal of Aircraft, Vol. 21, No. 8, 1984.

${ }^{11}$ Ricketts, R. H., Sandford, M. C., Seidel, D. A., and Watson, J. J., "Transonic Pressure Distributions on a Rectangular Supercritical Wing Oscillating in Pitch,” NASA TM 1983-84616, March 1983.

${ }^{12}$ Ricketts, R. H., Sandford, M. C., Watson, J. J., and Seidel, D. A., "Subsonic and Transonic Unsteady and Steady-Pressure Measurements on a Rectangular Supercritical Wing Oscillated in Pitch,” NASA TM 1984-85765, Nov. 1984.

${ }^{13}$ Bennett, R. M. and Walker, C. E., "Computational Test Cases for a Rectangular Supercritical Wing Undergoing Pitching Oscillations," NASA TM 1984-209130, April 1999.

${ }^{14}$ Wieseman, C. and Bennett, R., "Wall boundary layer measurements for the NASA Langley Transonic Dynamics Tunnel," NASA TM 200721867, 2007.

${ }^{15}$ Harris, C., "NASA supercritical airoils: a matrix of family-related airfoils," NASA TP 2969, 1990.

${ }^{16}$ Harris, C., "Aerodynamic characteristics of a 14-percent-thick NASA supercritical airfoil designed for a normal-force coefficient of 0.7 ," NASA TM X-72712, 1975.

${ }^{17}$ Lee, B. and Ohman, L., "Unsteady pressures and forces during transonic buffeting of a supercritical airfoil," Journal of Aircraft, Vol. 21, No. 6, pp. 439-441, June, 1986.

${ }^{18}$ Hurley, F., Spaid, F., Roos, R., Stivers, Jr., L., and Banettini, A., "Detailed transonic flow field measurements about a supercritical airoil section," NASA TM X-3244, 1975.

${ }^{19}$ Spalart, P. R. and Allmaras, S. R., "A One-Equation Turbulence Model for Aerodynamic Flows,” La Recherche Aerospatiale, No. 1, 1994, pp 5-21.

${ }^{20}$ Menter, F., "Two-Equation Eddy-Viscosity Turbulence Models for Engineering Applications,” AIAA Journal, Vol. 32, No. 8, 1994, pp 1598-1605

${ }^{21}$ Mavriplis, D., Vassberg, J., Tinoco, E., Mani, M., Brodersen, O., Eisfeld, B., Wahls, R., Morrison, J., Zuckuhr, T., Levy, D., and Murayama, M., "Grid quality and resolution issues from the Drag Prediction Workshop series," AIAA Paper 2008-930, Jan. 2008.

${ }^{22}$ Cummings, R., Morton, S., and McDaniel, D., "Experiences in accurately predicting time-dependent flows," Progress in Aerospace Sciences, Vol 44, No 4, pp.241-257, 2008.

${ }^{23}$ Mundell, A. and Mabey, D., "Pressure fluctuations caused by transonic shock/boundary-layer interaction," Aeronautical Journal, Vol. 90 , No. 897, Aug-Sept, 1986.

${ }^{24}$ Hillenherms, C., Schoeder, W., and Limberg, W., "Experimental investigation of a pitching airfoil in transonic flow," Applied Science and Technology, Vol. 8, pp. 583-590, 2004

${ }^{25}$ Campbell, R., personal communication, 2012.

${ }^{26}$ Anderson, J. J., Fundamentals of Aerodynamics, McGraw Hill Book Company, 1984.

${ }^{27}$ Bendat, J. and Piersol, A., Random data: Analysis and measurement procedures, John Wiley \& Sons, New York, 1971.

${ }^{28}$ Bendat, J. and Piersol, A., Engineering applications of correlation and spectral analysis, John Wiley \& Sons, New York, 1980.

${ }^{29} \mathrm{Heeg}, \mathrm{J}$. and Wieseman, C., "System identification and uncertainty quantification using orthogonal excitations and the Semi-span SuperSonic Transport (S4T) model," AIAA paper, presented at the 53 ${ }^{\text {rd }}$ AIAA Structural Dynamics and Materials Conference, Honolulu, April 2012.

${ }^{30}$ Schoukens, J., Rolain, Y., and Pentelon, R., "Analysis of windowing/leakage effects in frequency response function measurements," Automatica, 2011, Vol. 42, pp. 27-38, 2006.

${ }^{31}$ Morrison, J., "Statistical Analysis of CFD Solutions from the Fourth AIAA Drag Prediction Workshop," AIAA Paper 2010-4673, 2010.

${ }^{32}$ Rumsey, C., Slotnik, J., Long, M., Stuever, R., and Wayman, T., "Summary of the First AIAA CFD High-Lift Prediction Workshop," J. Aircraft, Vol. 48, No. 6, 2011.

${ }^{33}$ Olsen, J. J., Lambourne, N., et al., "Compendium of unsteady aerodynamic measurements,” AGARD R-702, Aug. 1999.

${ }^{34}$ Ruiz-Calavera, L. et al., "Verification and validation data for computational unsteady aerodynamics," RTO TR-26, Oct. 2000, Report of the Applied Vehicle Technology Panel (AVT) Task Group AVT-010. 


\section{Appendix A}

This appendix contains comparisons of the steady-state calculations of all analysts compared with the experimental unforced data at $\mathrm{M}=0.825, \alpha=2 \mathrm{deg}$ for all span stations.

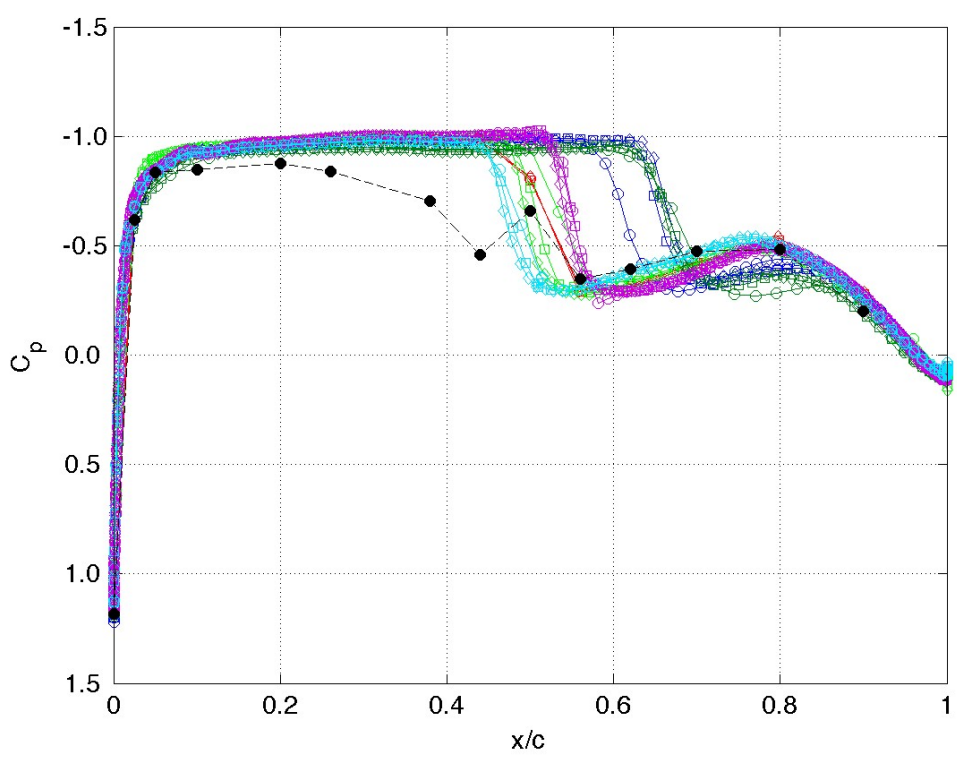

- A, Coarse
$\square$ A, Medium
- A, Fine
- B, Coarse
- B, Medium
- B, Fine
C, Coarse
C, Medium
C, Fine
D, Coarse
- D, Medium
- D, Fine
E, Coarse
E, Medium
E, Fine
F, Coarse
F, Medium
F, Fine
Experimental

(a) Upper Surface.

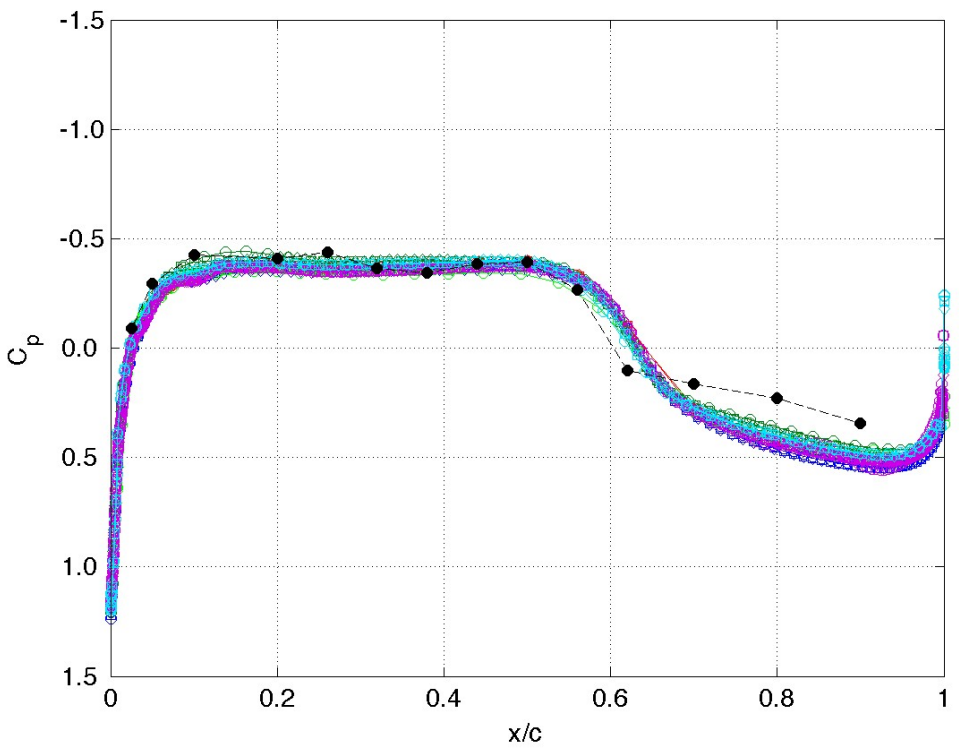

(b) Lower surface.

Figure 29. Unforced, $\alpha=2 \mathrm{deg}, \eta=0.309$. 


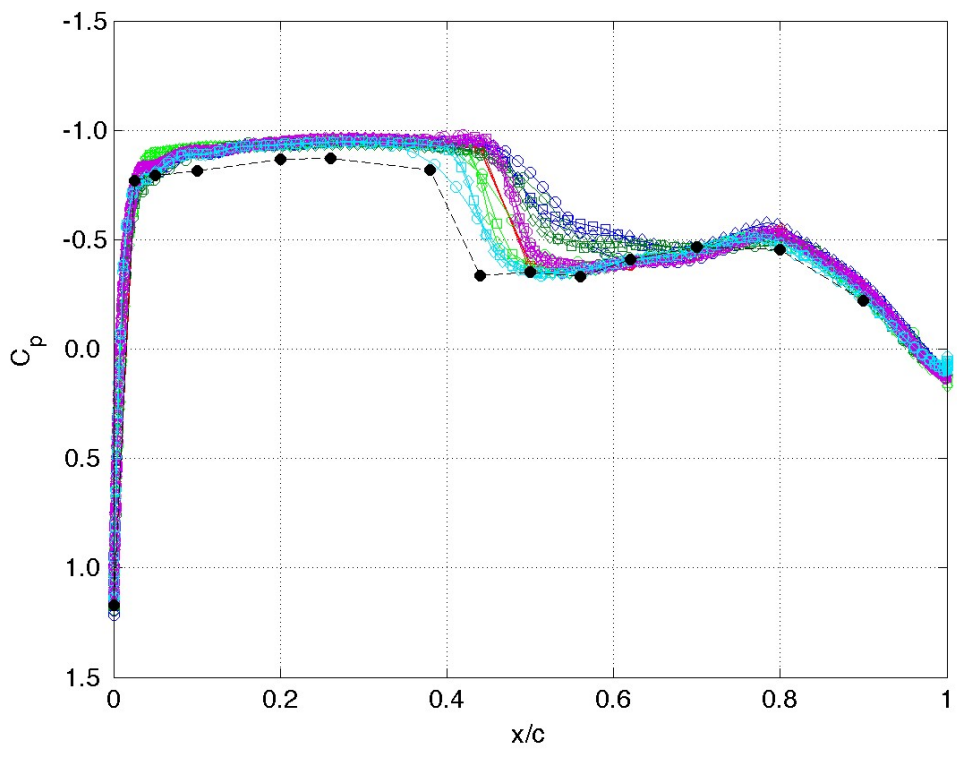

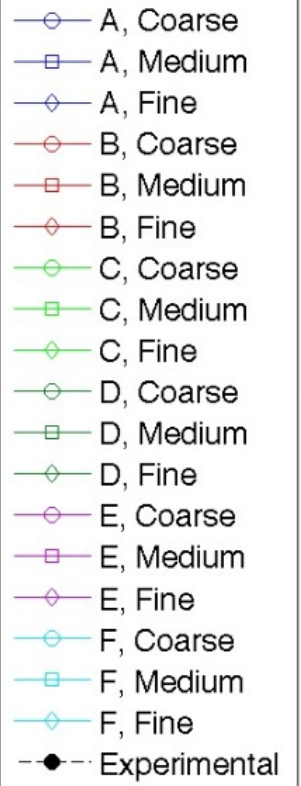

(a) Upper Surface.

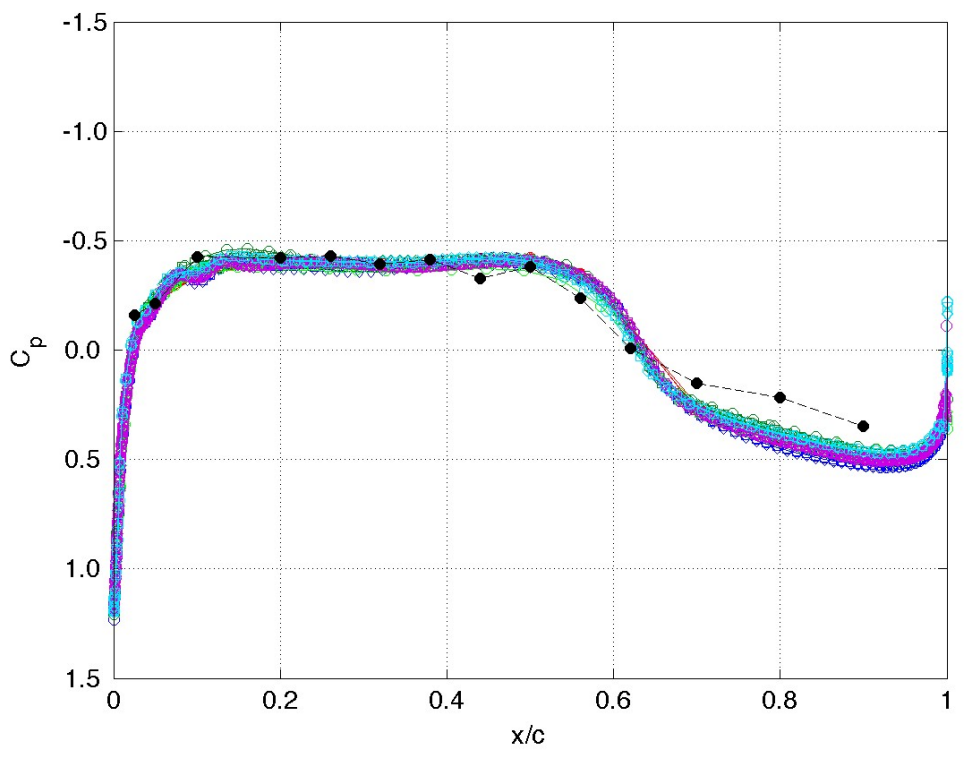

(b) Lower surface.

Figure 30. Unforced, $\alpha=2 \mathrm{deg}, \eta=0.588$. 


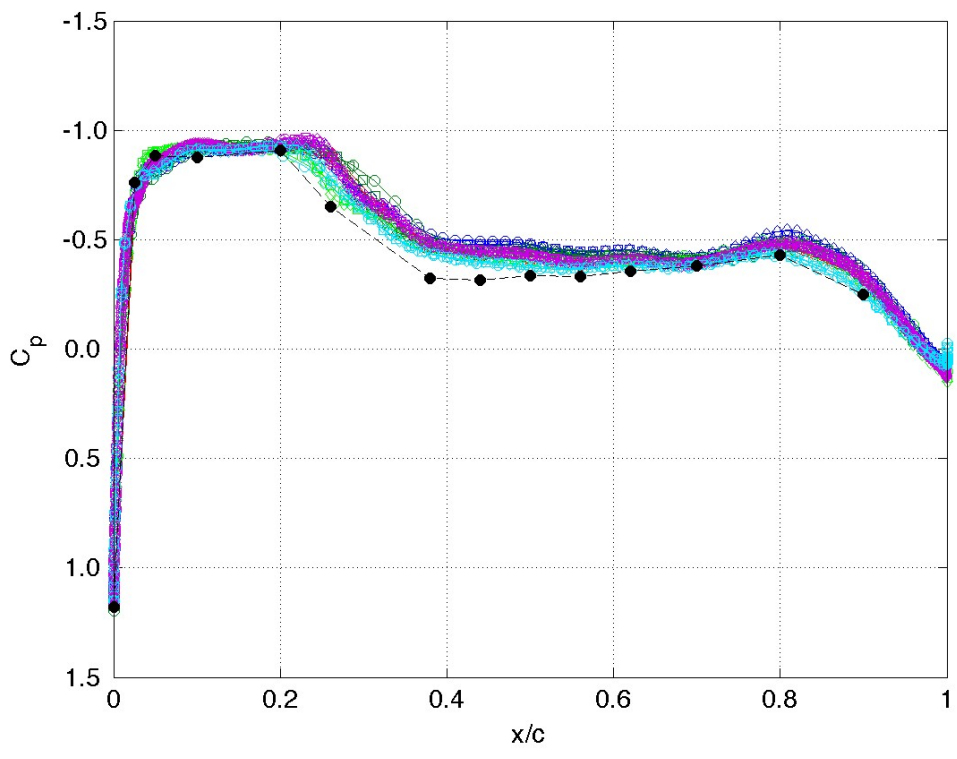

\begin{tabular}{|c}
- A, Coarse \\
$\square$ A, Medium \\
- A, Fine \\
- B, Coarse \\
- B, Medium \\
- B, Fine \\
C, Coarse \\
$\square$ C, Medium \\
C, Fine \\
- D, Coarse \\
- D, Medium \\
- D, Fine \\
- E, Coarse \\
E, Medium \\
E, Fine \\
F, Coarse \\
F, Medium \\
F, Fine \\
- Experimental
\end{tabular}

(a) Upper Surface.

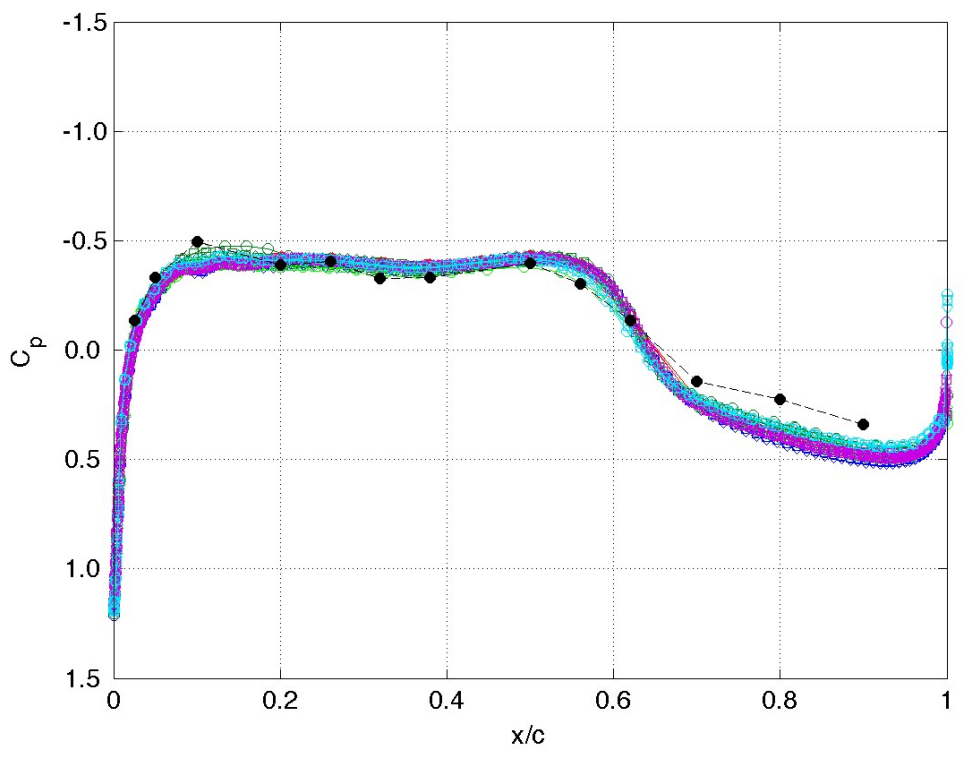

(b) Lower surface.

Figure 31. Unforced, $\alpha=2$ deg, $\eta=0.809$. 


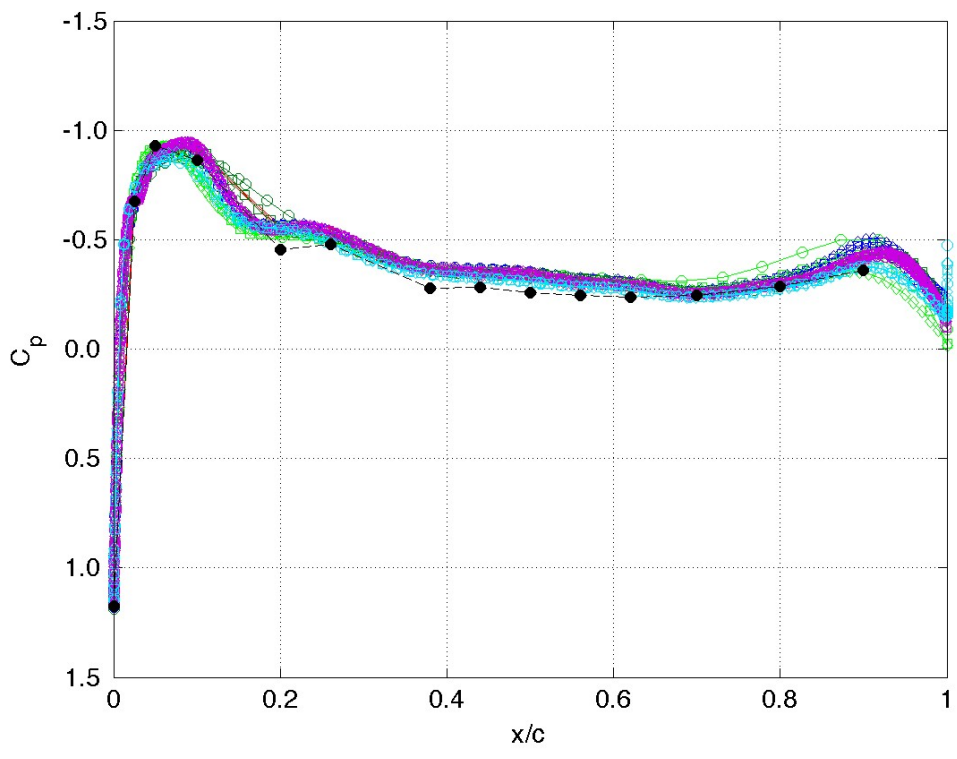

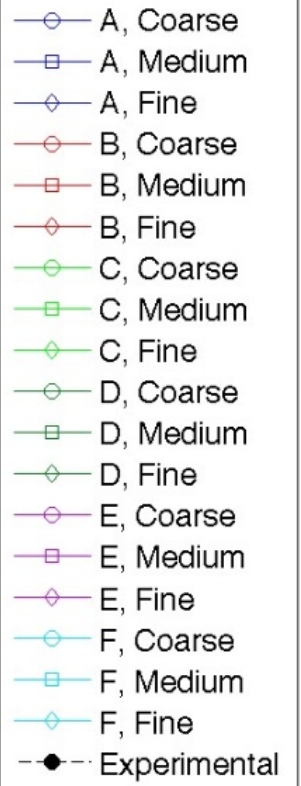

(a) Upper Surface.

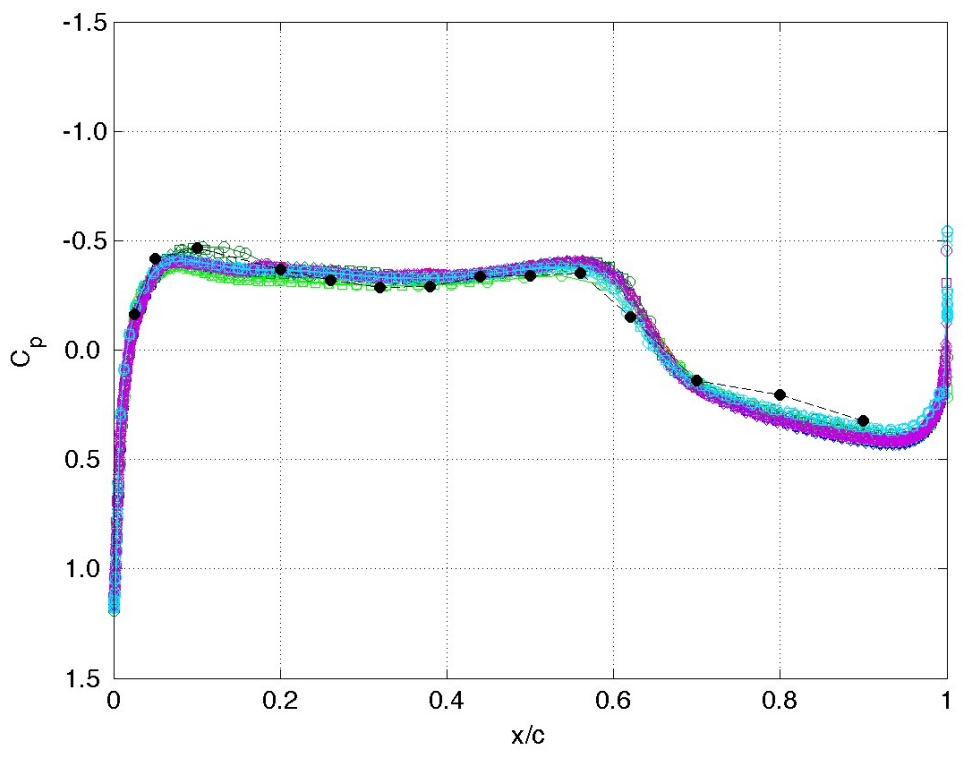

(b) Lower surface.

Figure 32. Unforced, $\alpha=2 \mathrm{deg}, \eta=0.951$. 


\section{Appendix B}

This appendix contains comparisons of the steady-state calculations of all analysts compared with the experimental unforced data at $\mathrm{M}=0.825, \alpha=4 \mathrm{deg}$ for all span stations.

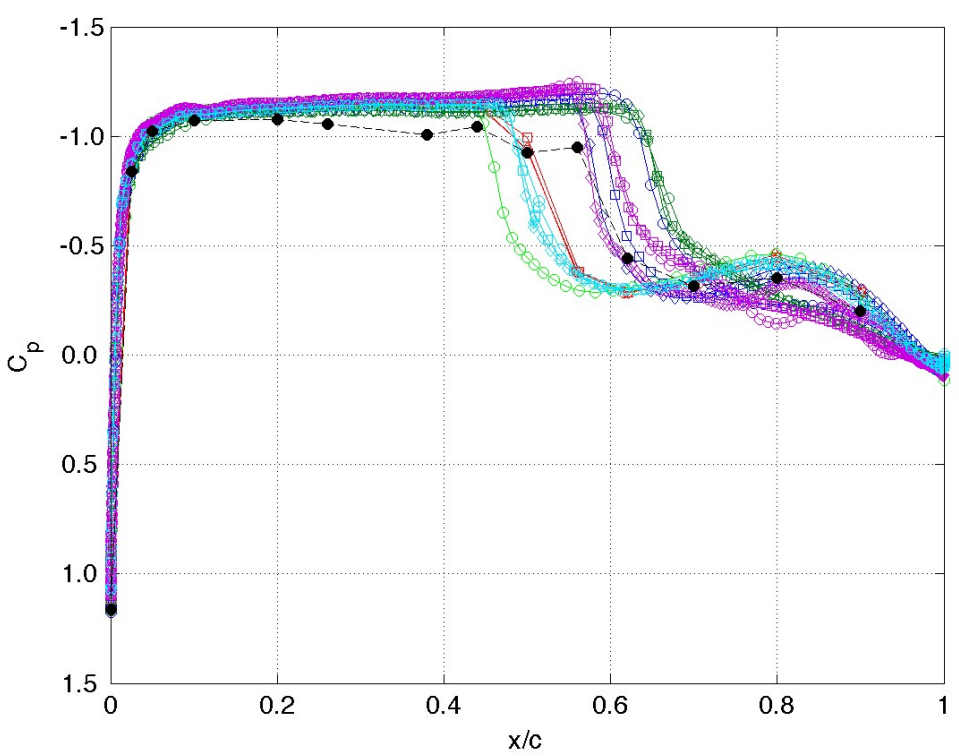

\begin{tabular}{|l}
\hline- A, Coarse \\
- A, Medium \\
- A, Fine \\
- B, Coarse \\
- B, Medium \\
- B, Fine \\
- C, Medium \\
- D, Coarse \\
- D, Medium \\
- D, Fine \\
- E, Coarse \\
- E, Medium \\
- E, Fine \\
F, Coarse \\
F, Medium \\
- F, Fine \\
- Experimental
\end{tabular}

(a) Upper Surface.

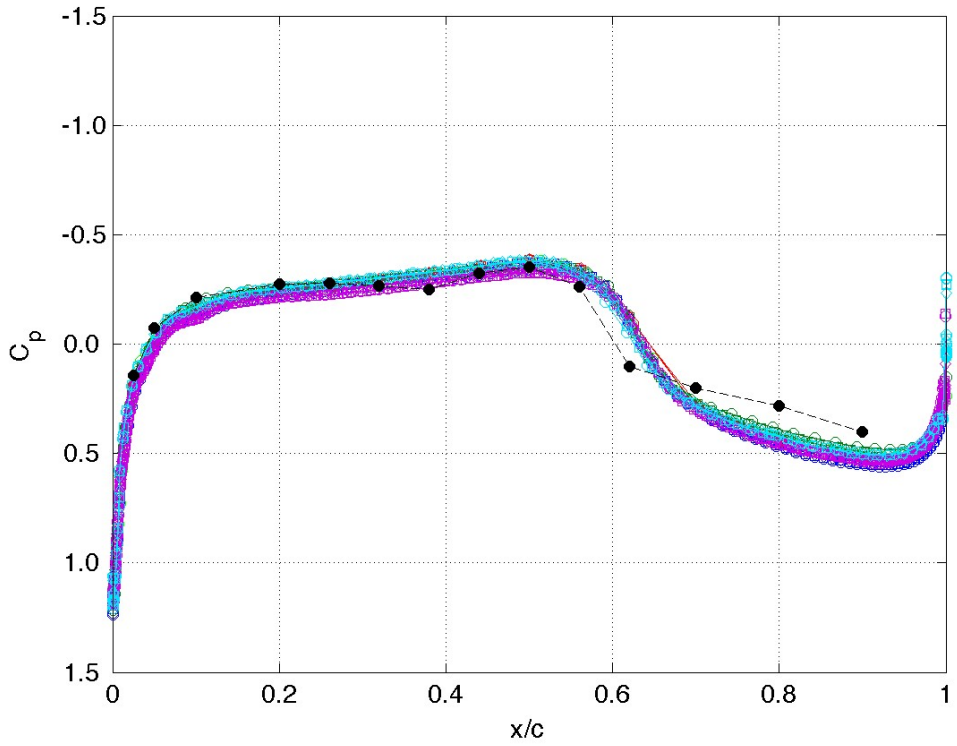

(b) Lower surface.

Figure 33. Unforced, $\alpha=4 \mathrm{deg}, \eta=0.309$. 


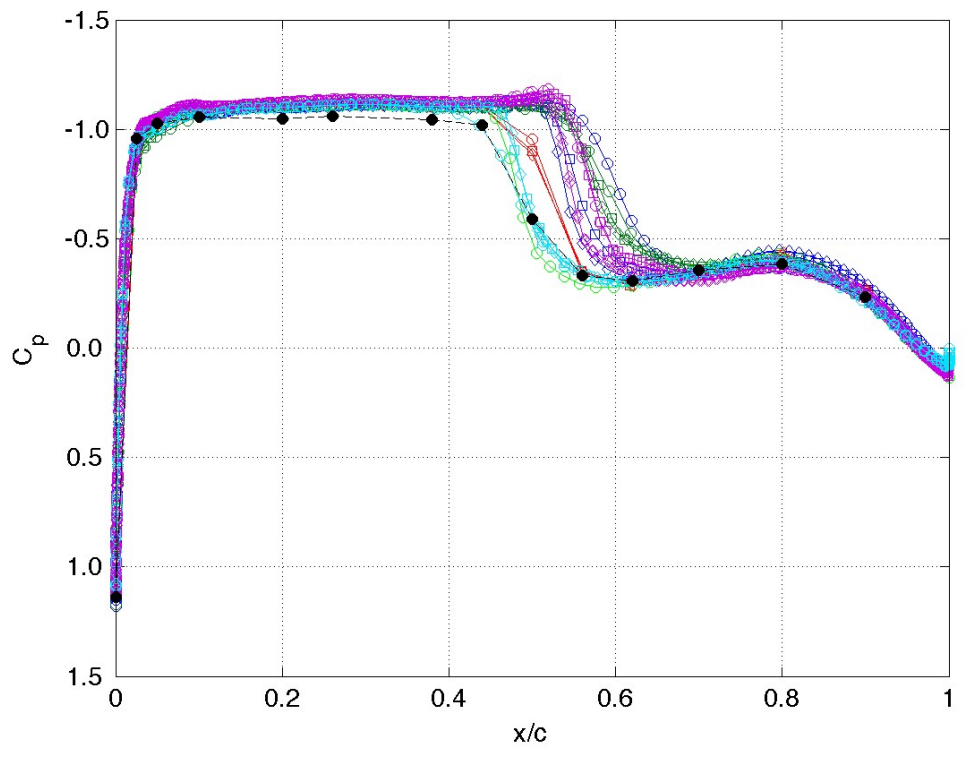

\begin{tabular}{|l|}
\hline- A, Coarse \\
- A, Medium \\
- A, Fine \\
- B, Coarse \\
- B, Medium \\
- B, Fine \\
- C, Medium \\
- D, Coarse \\
- D, Medium \\
- D, Fine \\
- E, Coarse \\
- E, Medium \\
- E, Fine \\
F, Coarse \\
F, Medium \\
F, Fine \\
- Experimental
\end{tabular}

(a) Upper Surface.

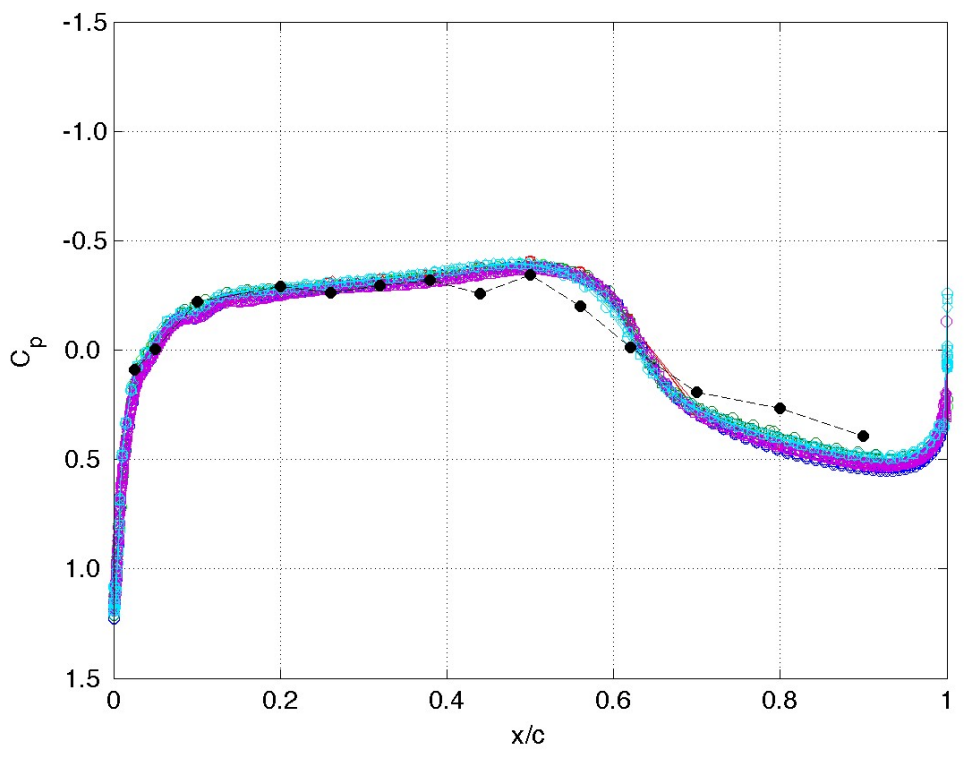

(b) Lower surface.

Figure 34. Unforced, $\alpha=4 \mathrm{deg}, \eta=0.588$. 


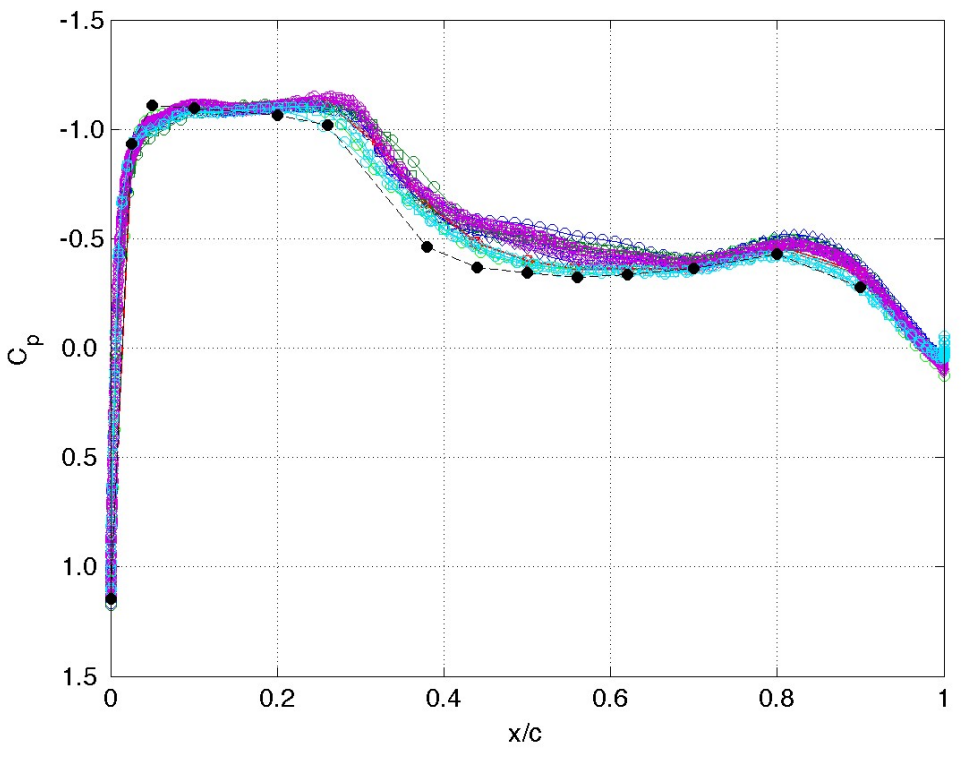

$-\because$ A, Coarse
- A, Medium
- A, Fine
- B, Coarse
- B, Medium
- B, Fine
C, Medium
- D, Coarse
- D, Medium
- D, Fine
- E, Coarse
- E, Medium
E, Fine
F, Coarse
F, Medium
F, Fine
- Experimental

(a) Upper Surface.

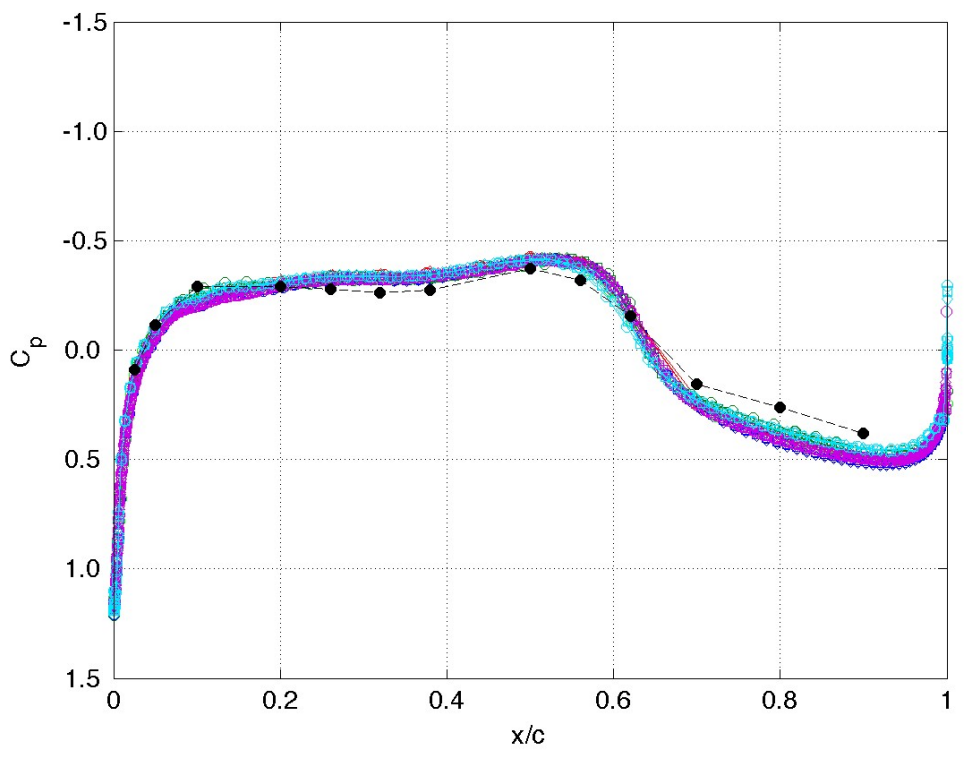

(b) Lower surface.

Figure 35. Unforced, $\alpha=4 \mathrm{deg}, \eta=0.809$. 


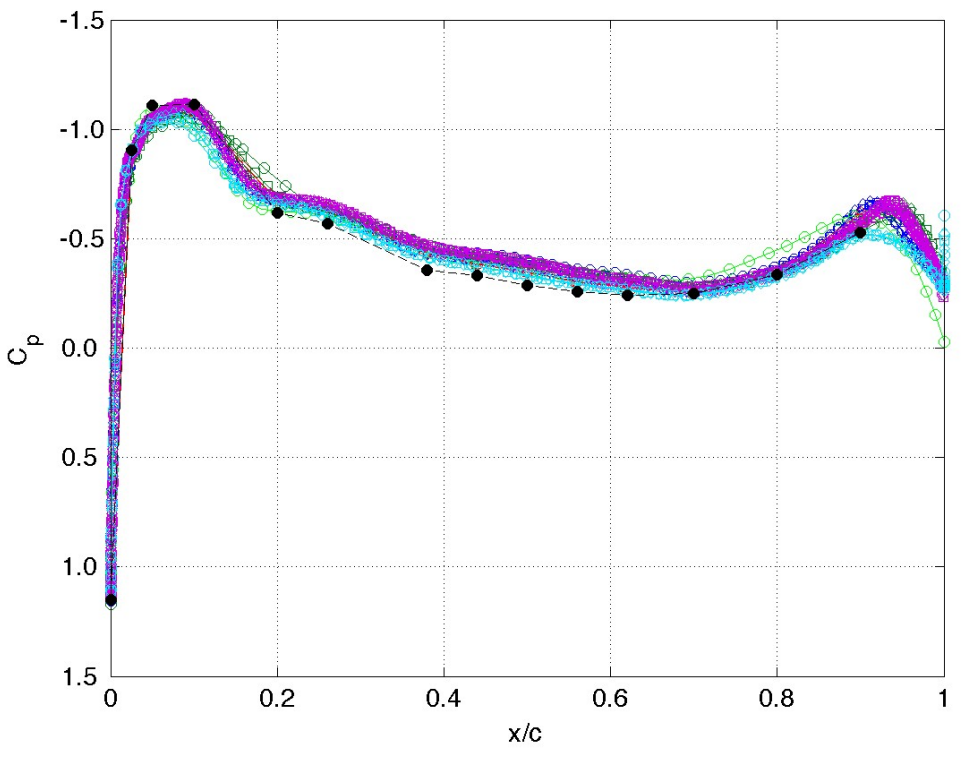

\begin{tabular}{|l|}
\hline- A, Coarse \\
- A, Medium \\
- A, Fine \\
- B, Coarse \\
- B, Medium \\
- B, Fine \\
- C, Medium \\
- D, Coarse \\
- D, Medium \\
- D, Fine \\
- E, Coarse \\
- E, Medium \\
- E, Fine \\
F, Coarse \\
F, Medium \\
F, Fine \\
- Experimental
\end{tabular}

(a) Upper Surface.

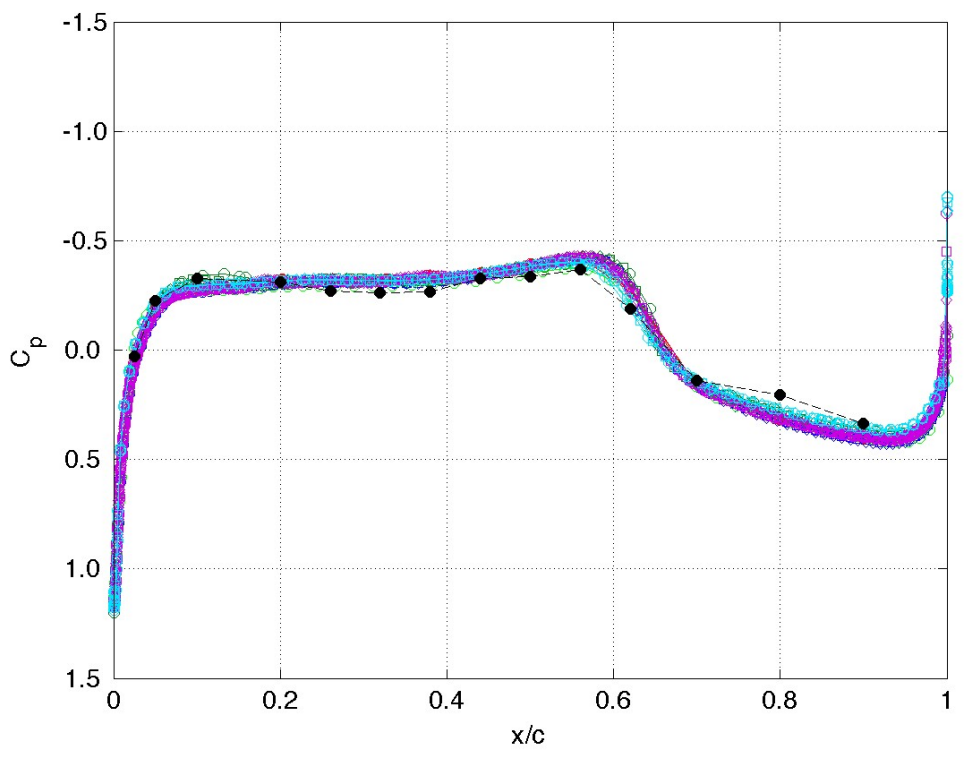

(b) Lower surface.

Figure 36. Unforced, $\alpha=4 \mathrm{deg}, \eta=0.951$. 


\section{Appendix C}

This appendix contains comparisons of the unsteady magnitude and phase from CFD calculations of all analysts compared with the experimental forced data at $\mathrm{M}=0.825, \alpha=2 \mathrm{deg}$, at frequency of $10 \mathrm{~Hz}$ at all span stations.

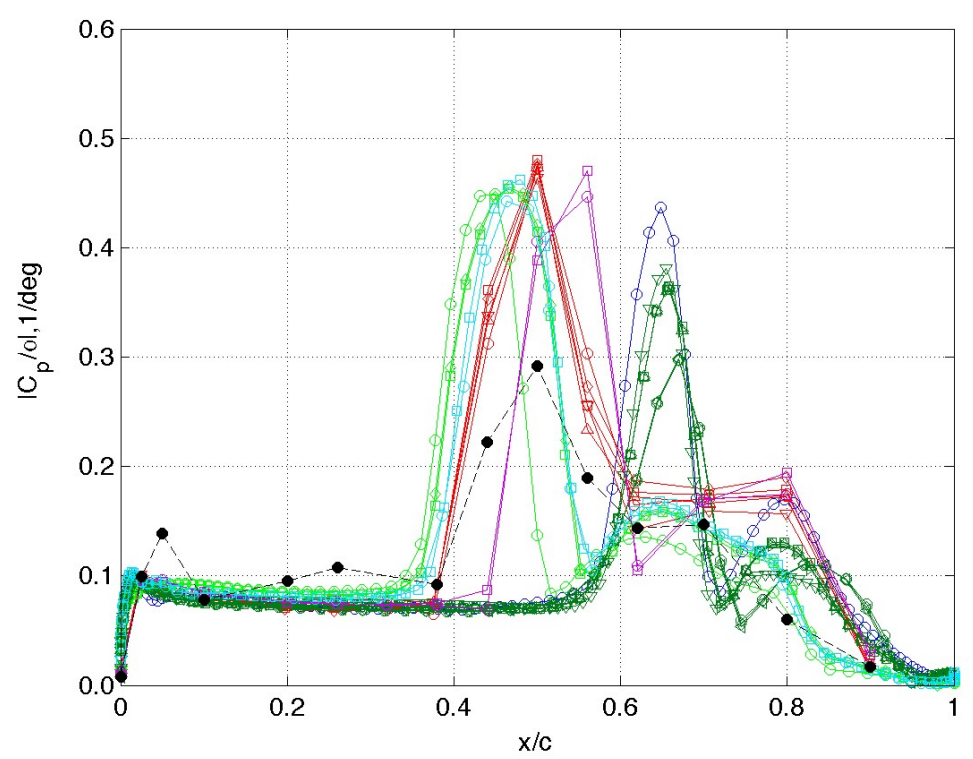

(a) Magnitude.

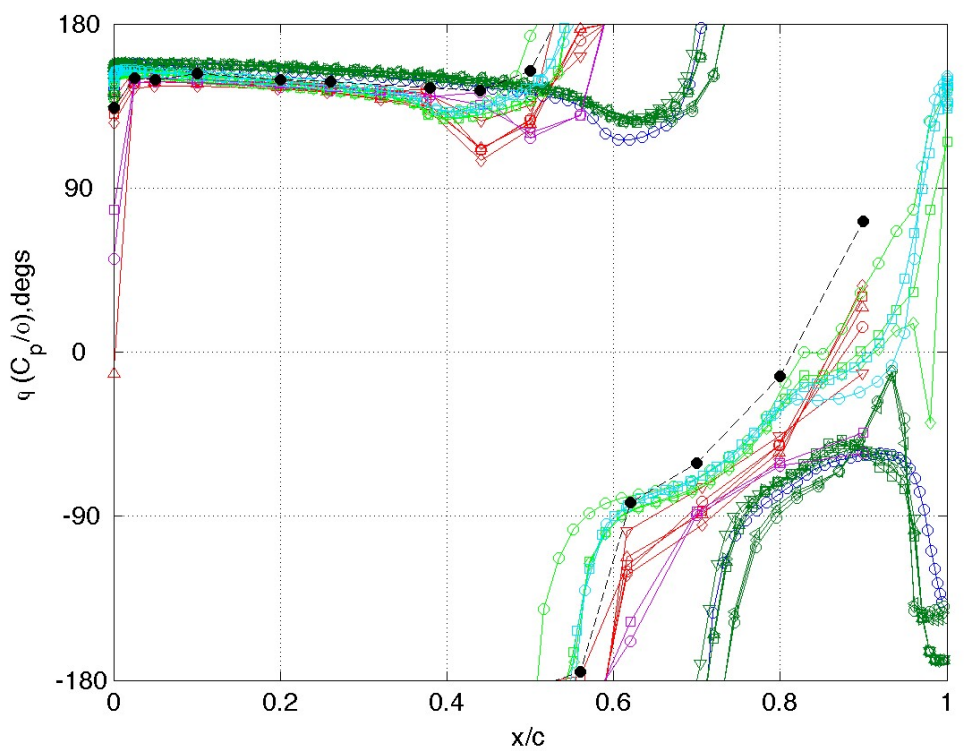

(b) Phase, deg.

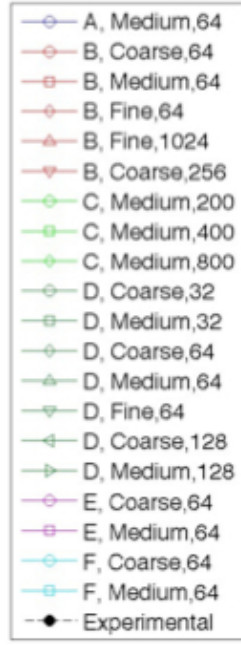

A, Medium, 64

- - B. Medium 64

- C. Medium, 200

- C. Medium, 400

- D. Coarse, 32

- D, Medium, 32

-D D. Fine, 64

-4- D. Coarse, 128

- E, Coarse 64

- E. Medium,64

F, Coarse, 64

- Experimental

Figure 37. Forced, $\alpha=2 \mathrm{deg}$, frequency $=10 \mathrm{~Hz}, \eta=0.309$. 

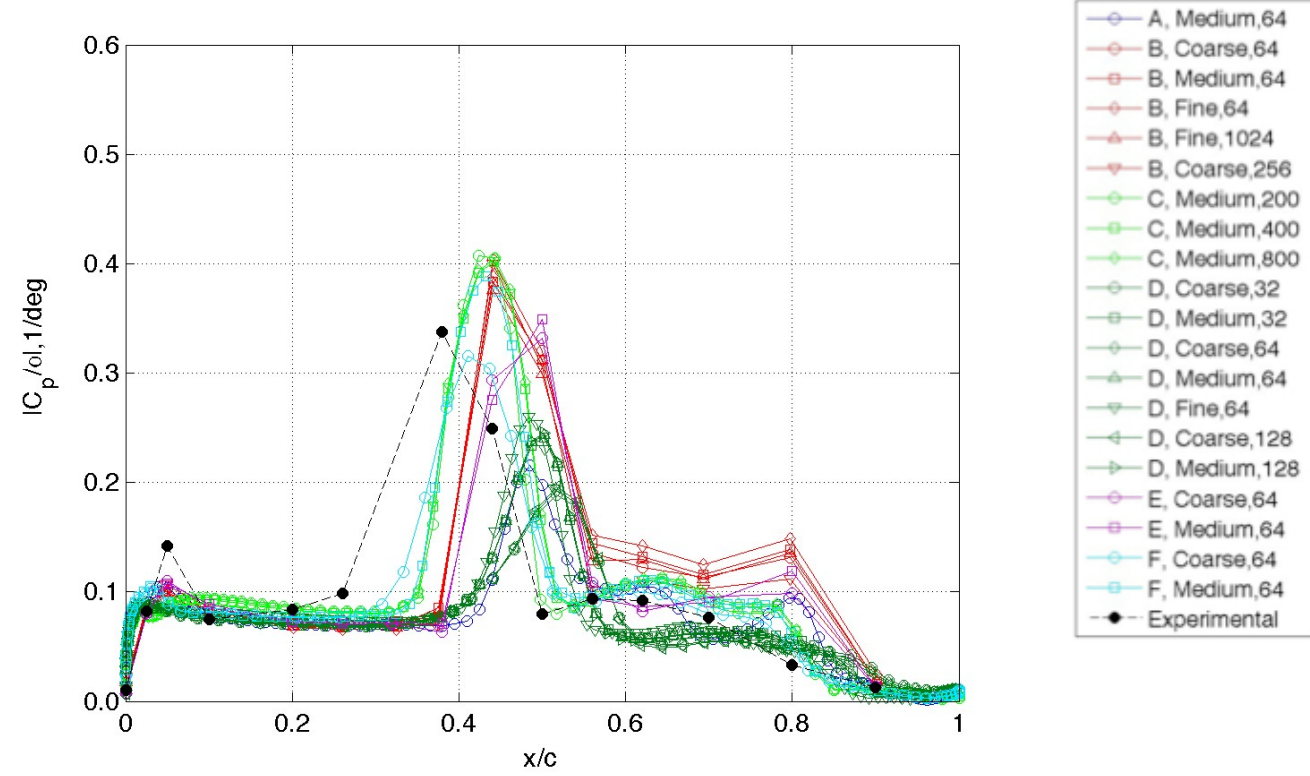

(a) Magnitude.

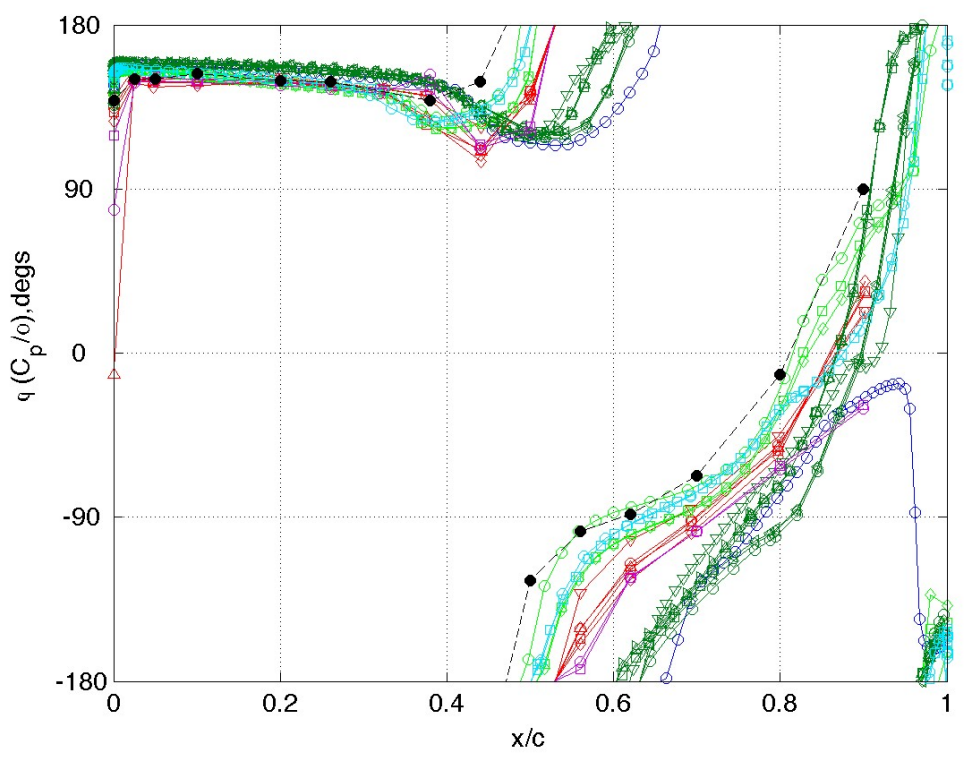

(b) Phase, deg.

Figure 38. Forced, $\alpha=2 \mathrm{deg}$, frequency $=10 \mathrm{~Hz}, \eta=0.588$. 

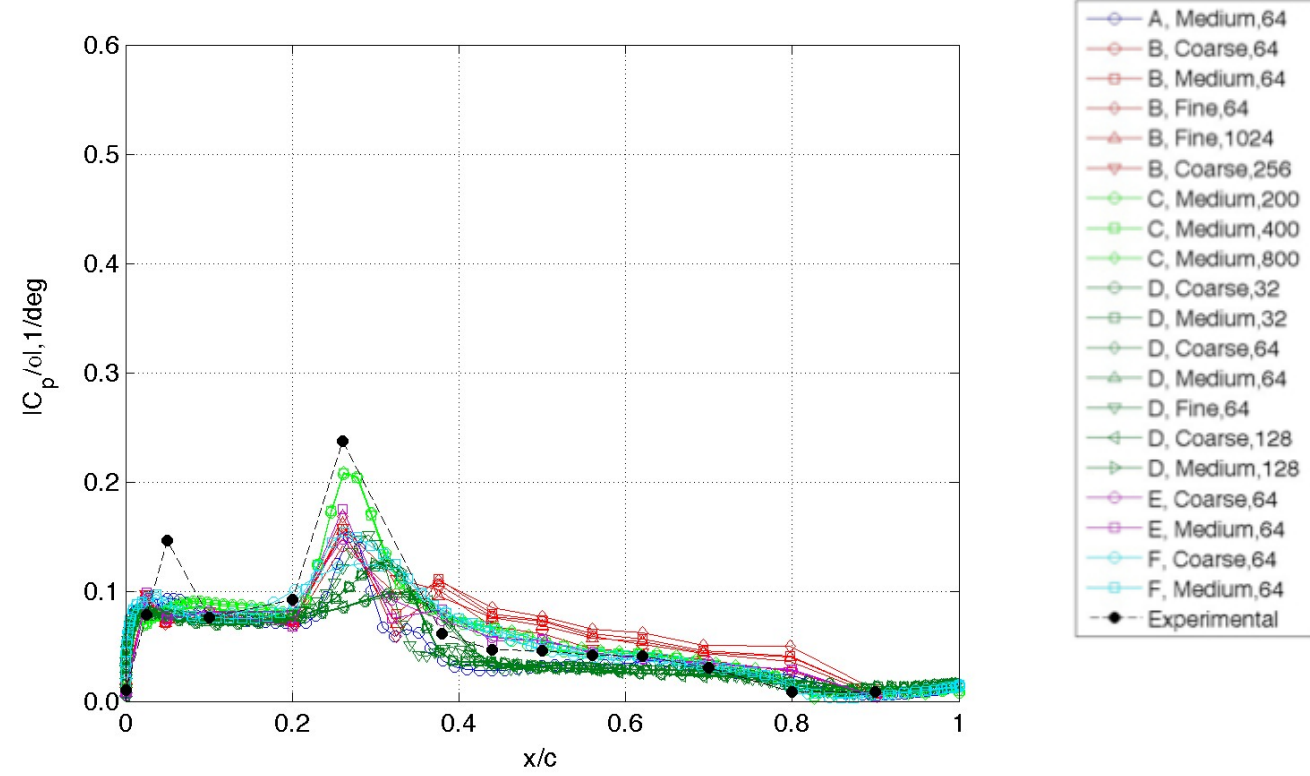

(a) Magnitude.

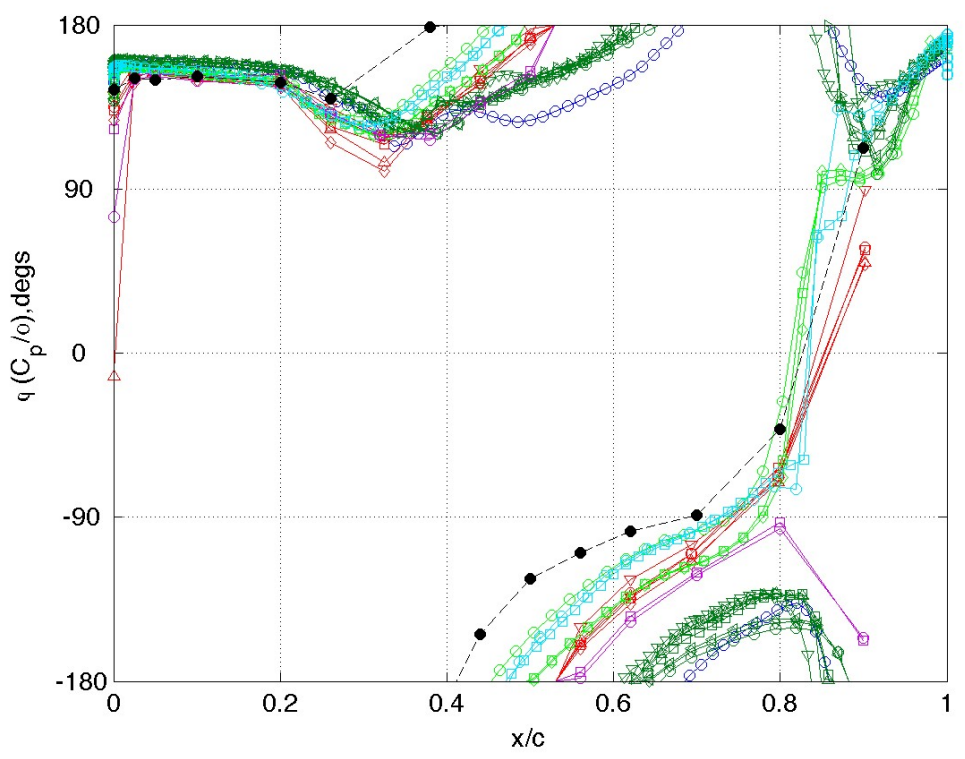

(b) Phase, deg.

Figure 39. Forced, $\alpha=2 \mathrm{deg}$, frequency $=10 \mathrm{~Hz}, \eta=0.809$. 

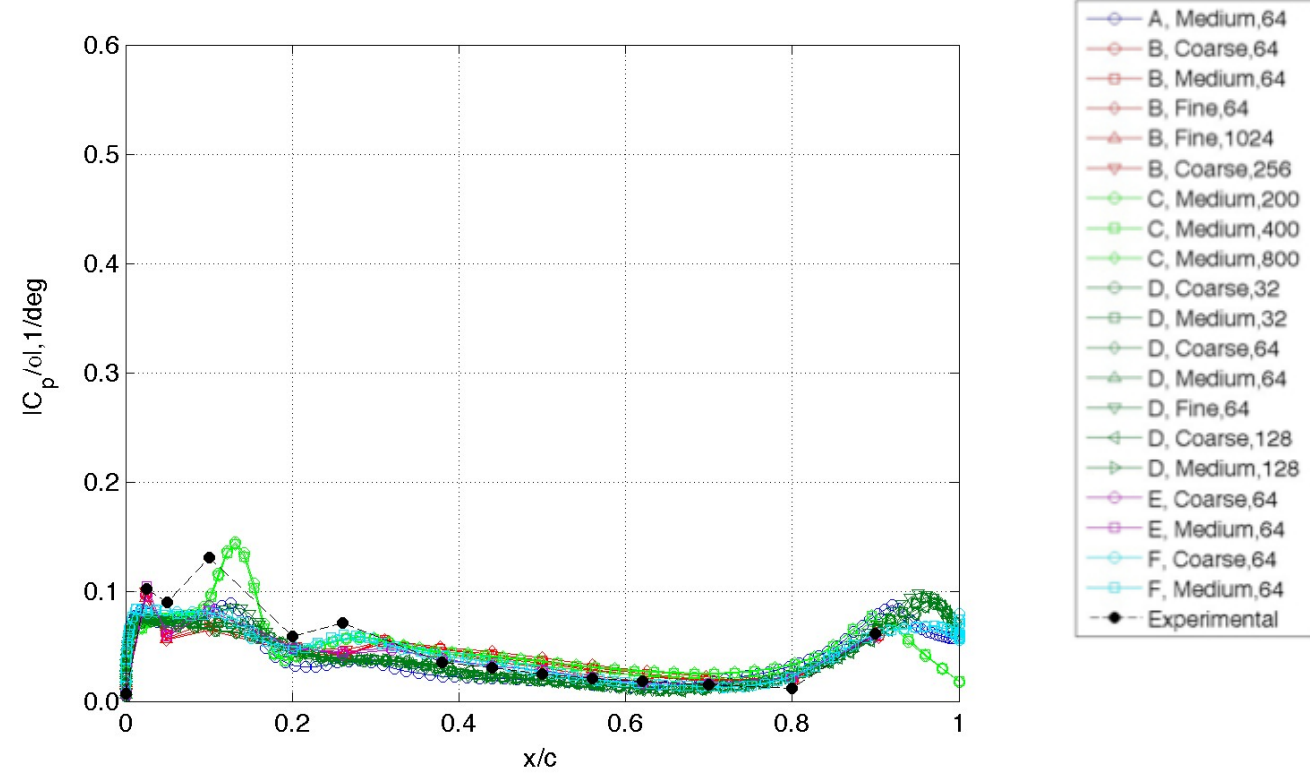

(a) Magnitude.

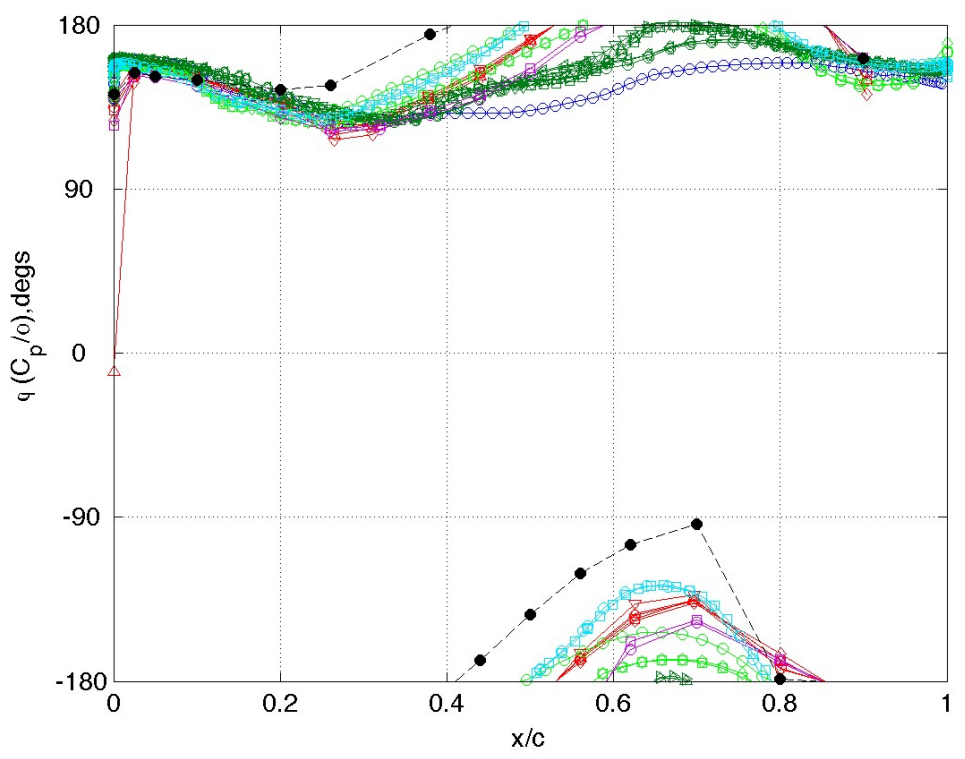

(b) Phase, deg.

Figure 40. Forced, $\alpha=2 \mathrm{deg}$, frequency $=10 \mathrm{~Hz}, \eta=0.951$. 

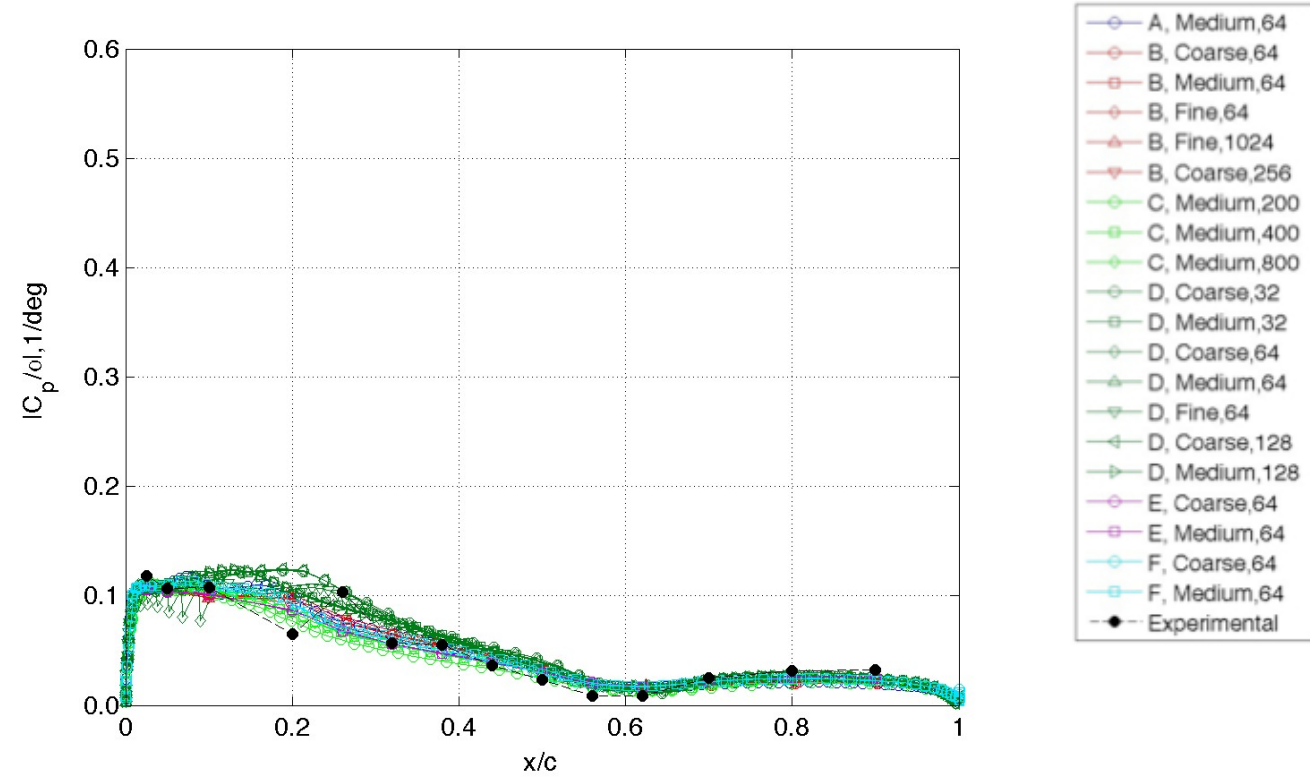

(a) Magnitude.

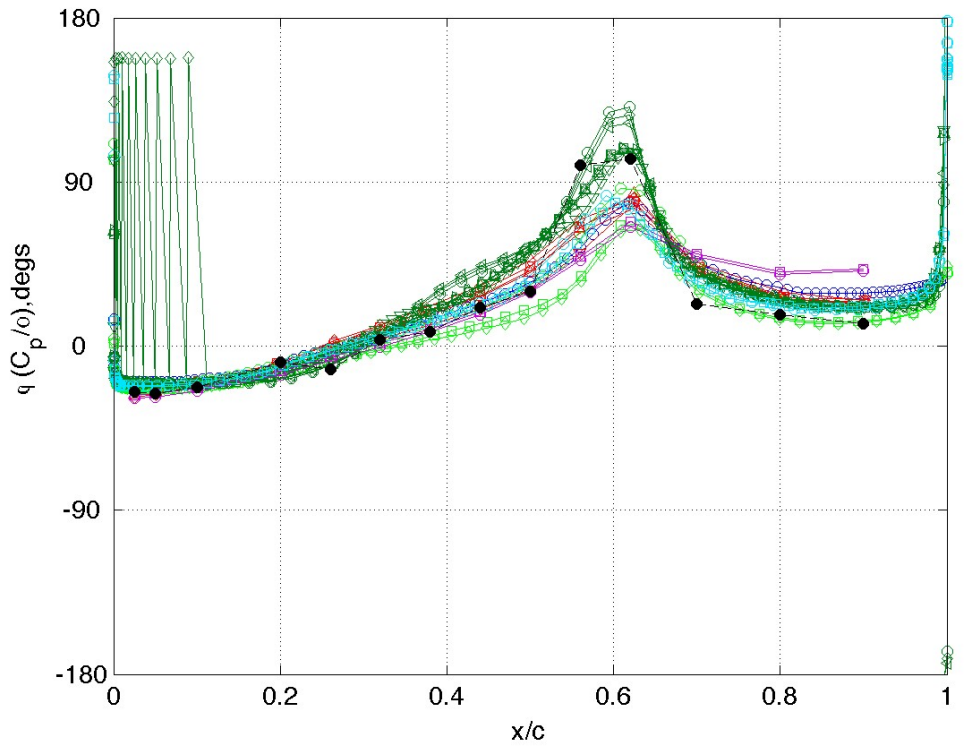

(b) Phase, deg.

Figure 41. Forced, $\alpha=2 \mathrm{deg}$, frequency $=10 \mathrm{~Hz}, \eta=0.309$. 

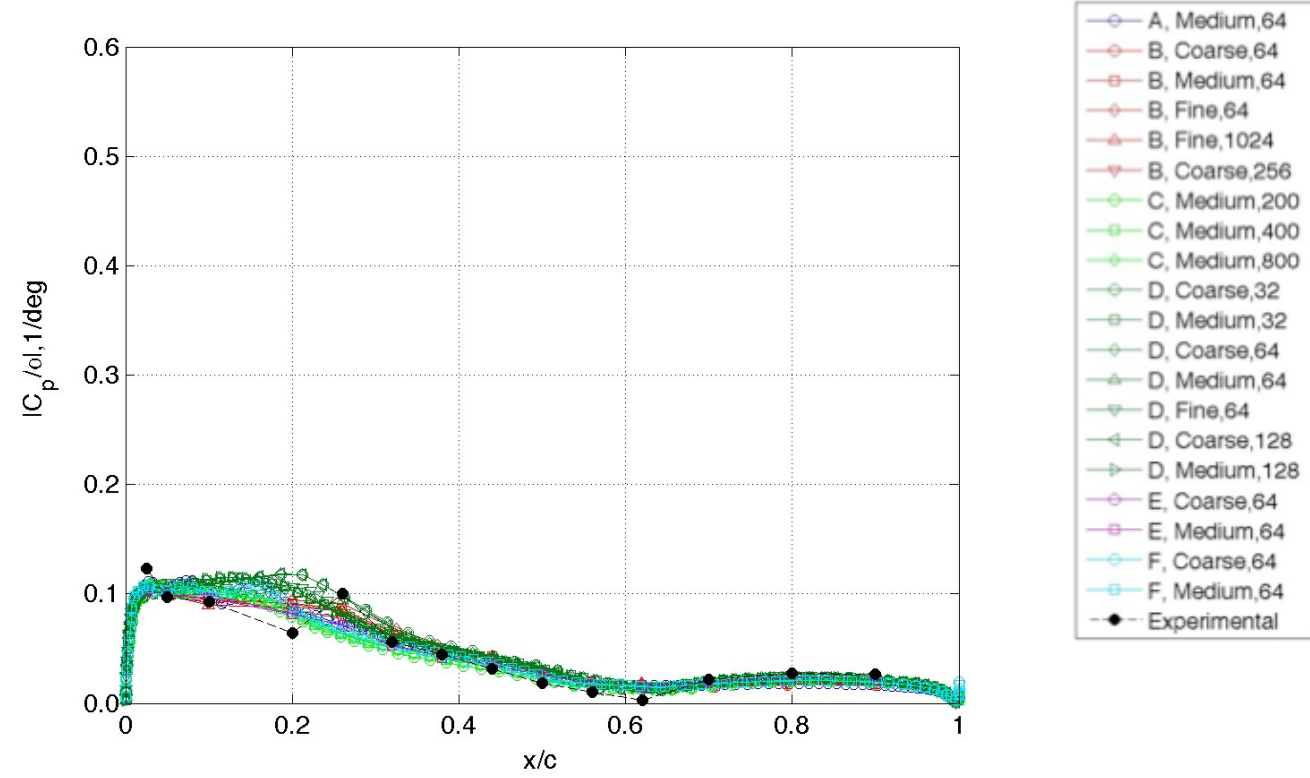

(a) Magnitude.

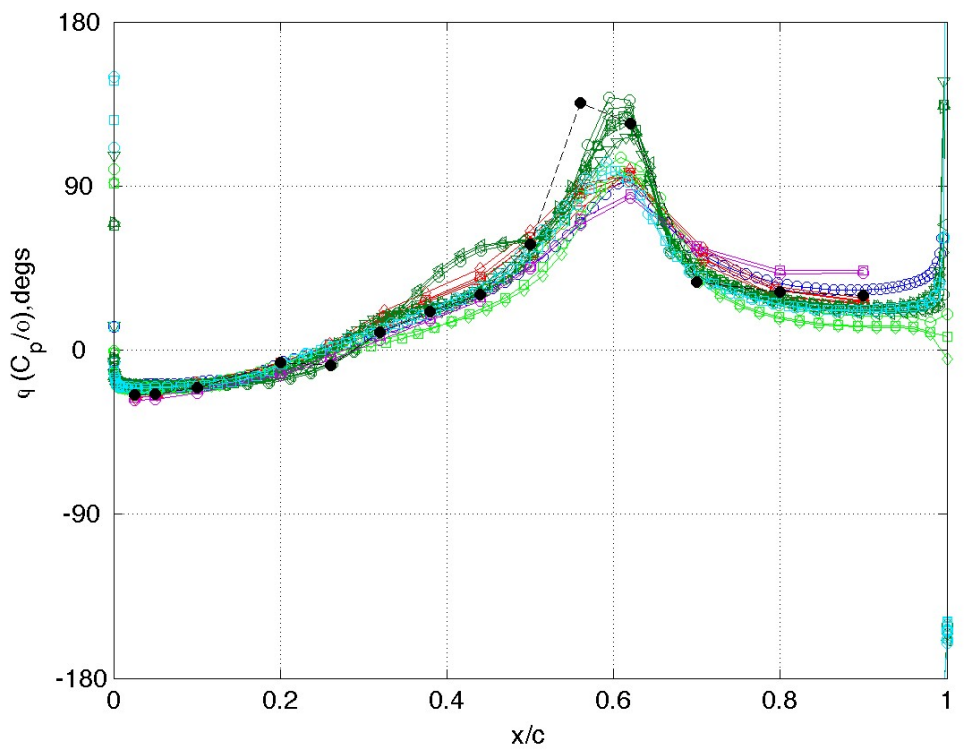

(b) Phase, deg.

Figure 42. Forced, $\alpha=2 \mathrm{deg}$, frequency $=10 \mathrm{~Hz}, \eta=0.588$. 

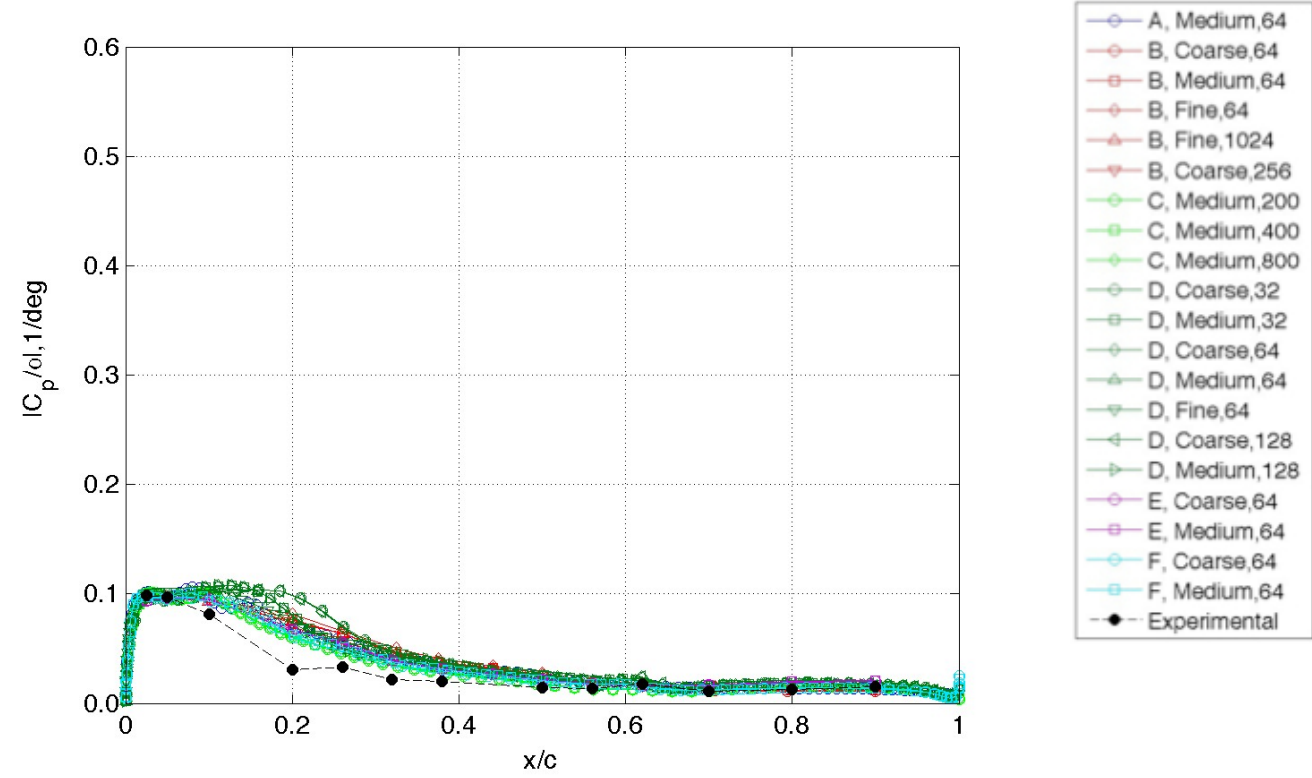

(a) Magnitude.

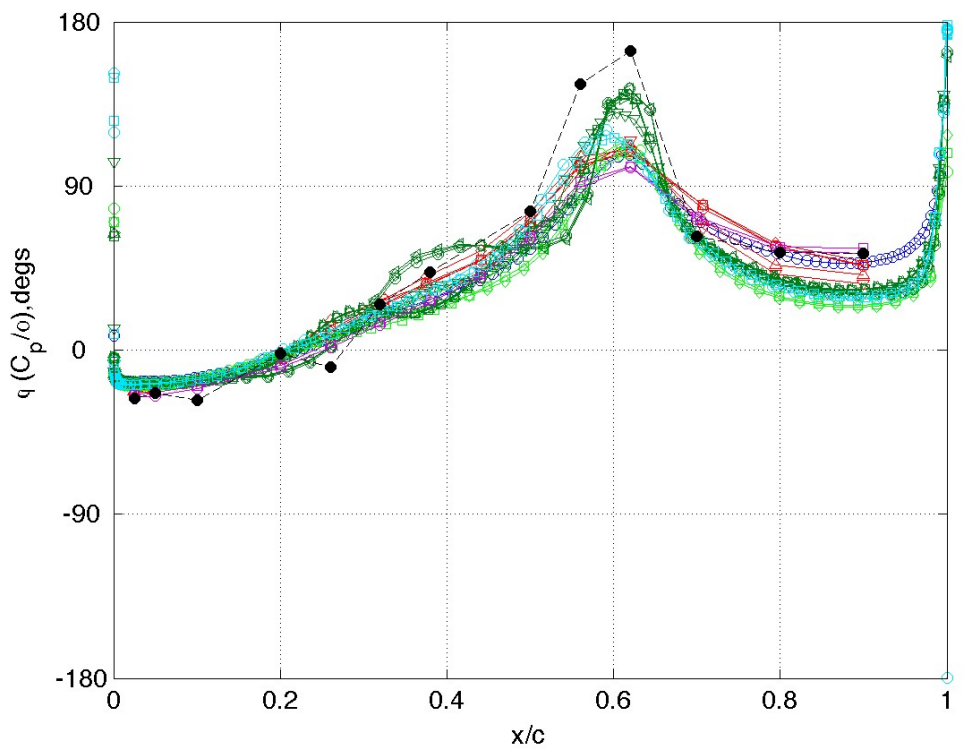

(b) Phase, deg.

Figure 43. Forced, $\alpha=2 \mathrm{deg}$, frequency $=10 \mathrm{~Hz}, \eta=0.809$. 

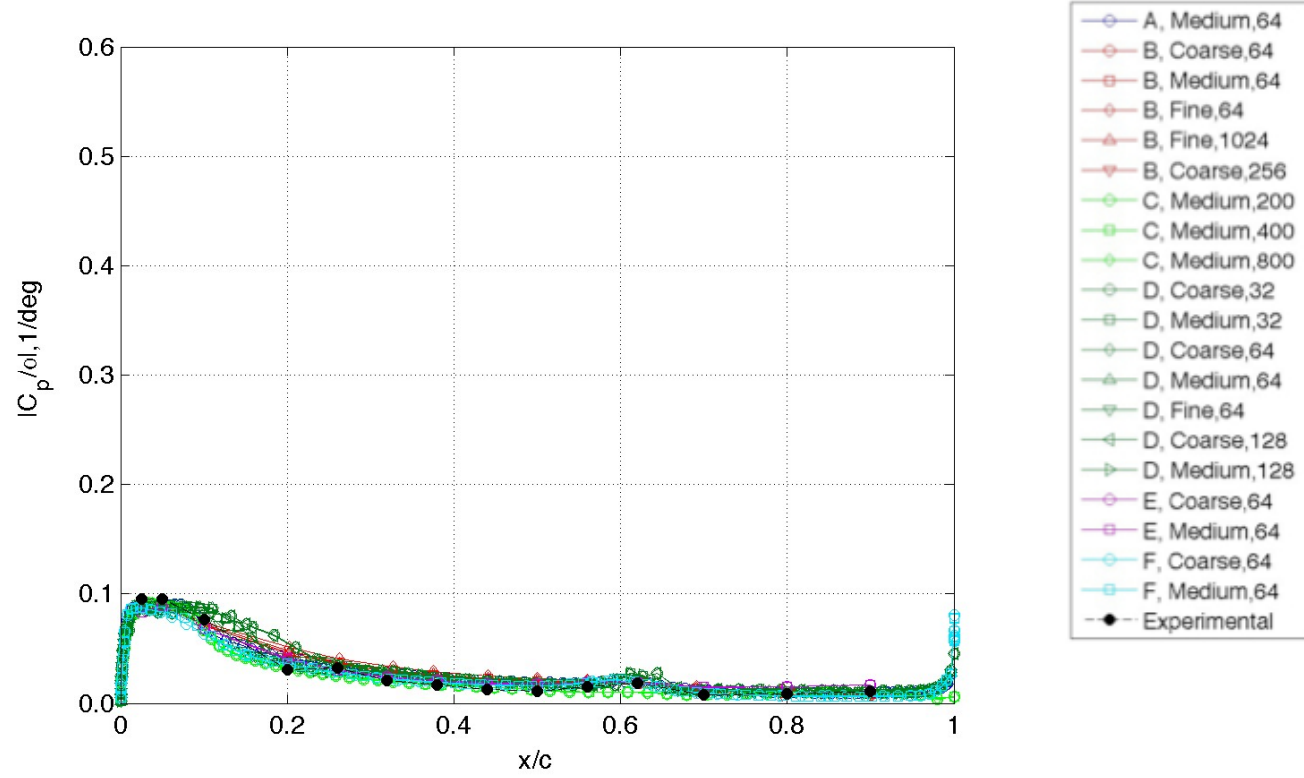

(a) Magnitude.

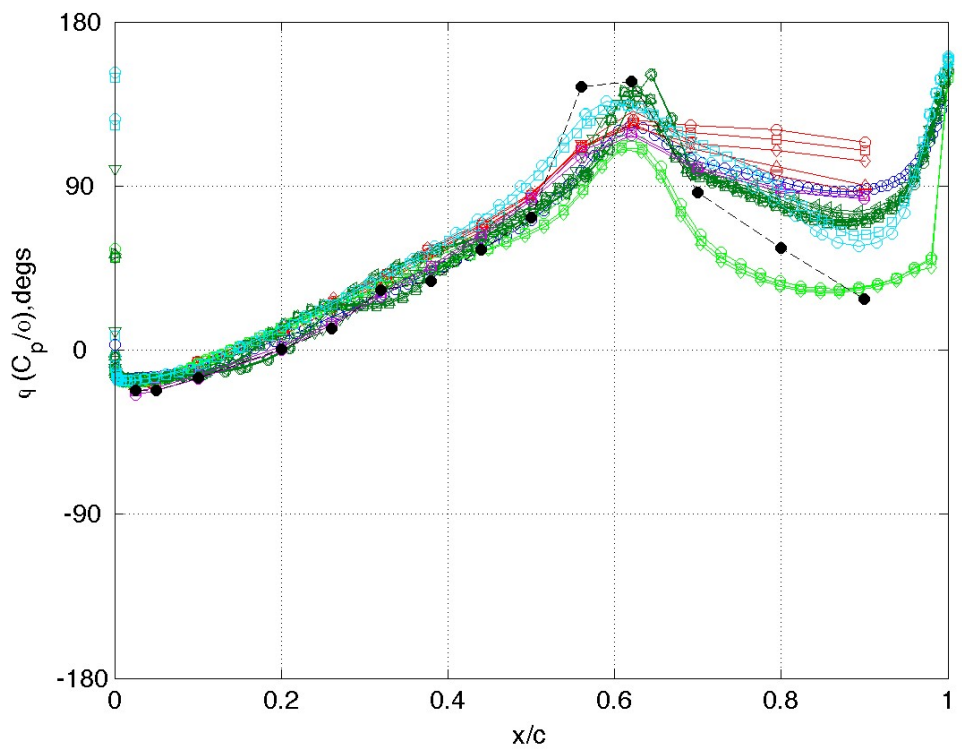

(b) Phase, deg

Figure 44. Forced, $\alpha=2 \mathrm{deg}$, frequency $=10 \mathrm{~Hz}, \eta=0.951$. 


\section{Appendix D}

This appendix contains comparisons of the unsteady magnitude and phase from CFD calculations of all analysts compared with the experimental forced data at $\mathrm{M}=0.825, \alpha=2 \mathrm{deg}$, at frequency of $20 \mathrm{~Hz}$ at all span stations.

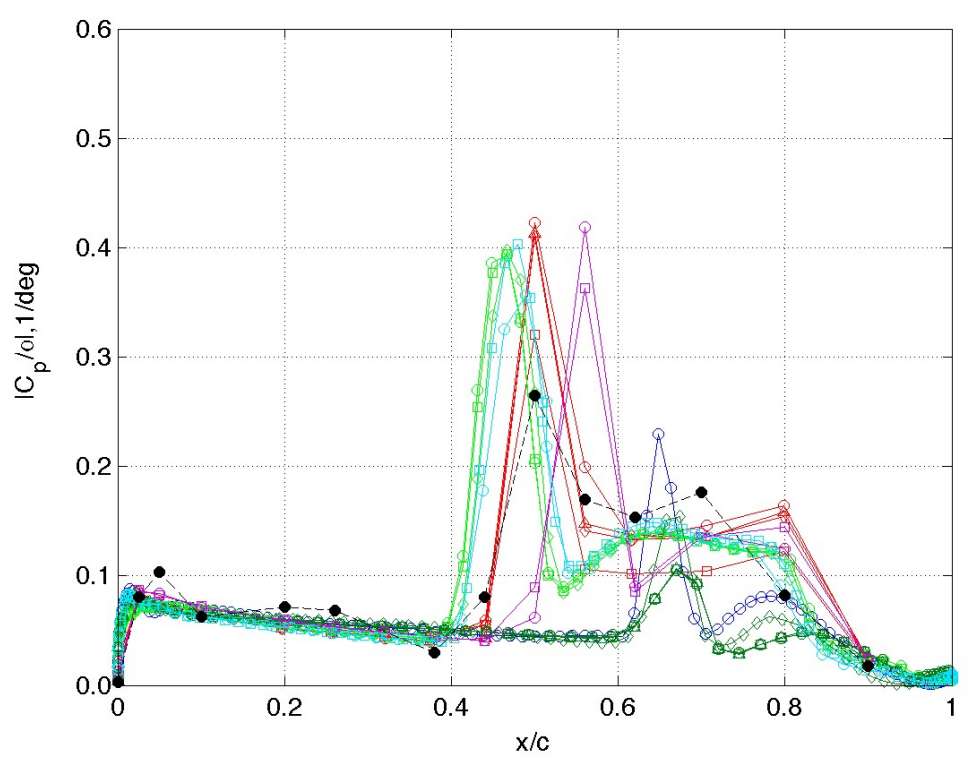

(a) Magnitude.

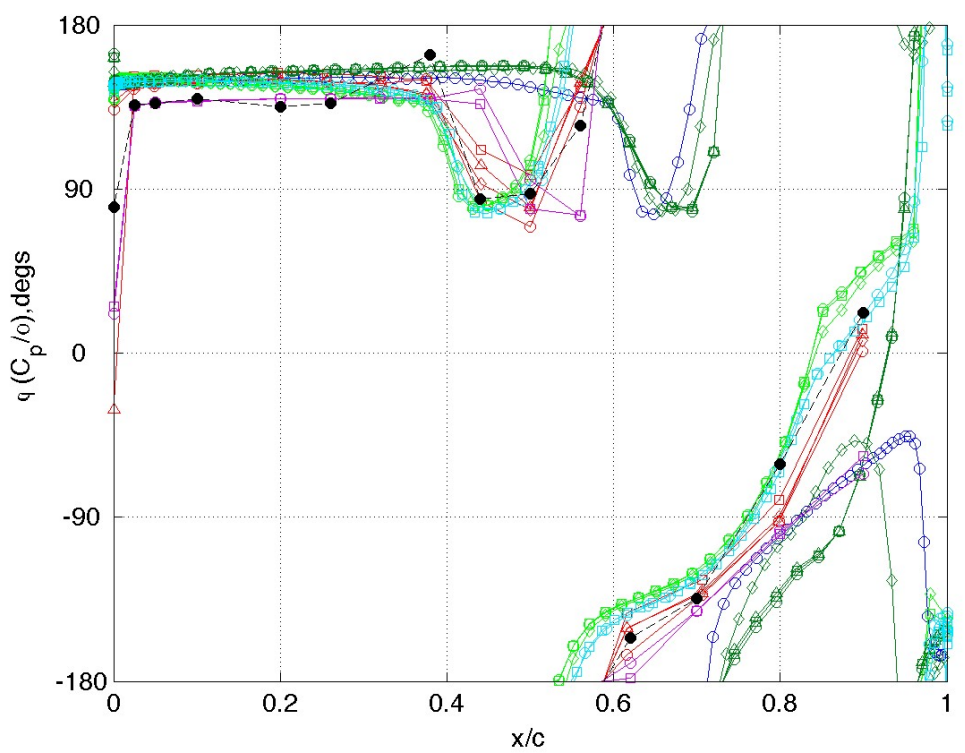

(b) Phase, deg.

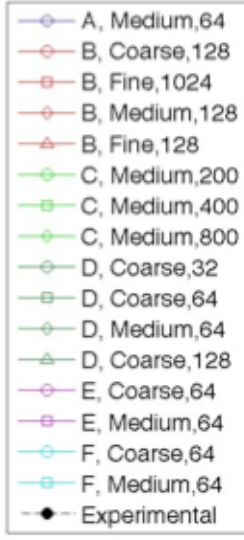

$-\mathrm{B}$, Coarse, 128

- - B. Fine, 1024

$\triangle \mathrm{B}$, Fine, 128

- C, Medium,200

- D D Coarse, 32

- D. Coarse,64

(2) E, Coarse, 64

, Medium, 64

- - Experim

Figure 45. Forced, $\alpha=2 \mathrm{deg}$, frequency $=20 \mathrm{~Hz}, \eta=0.309$ 

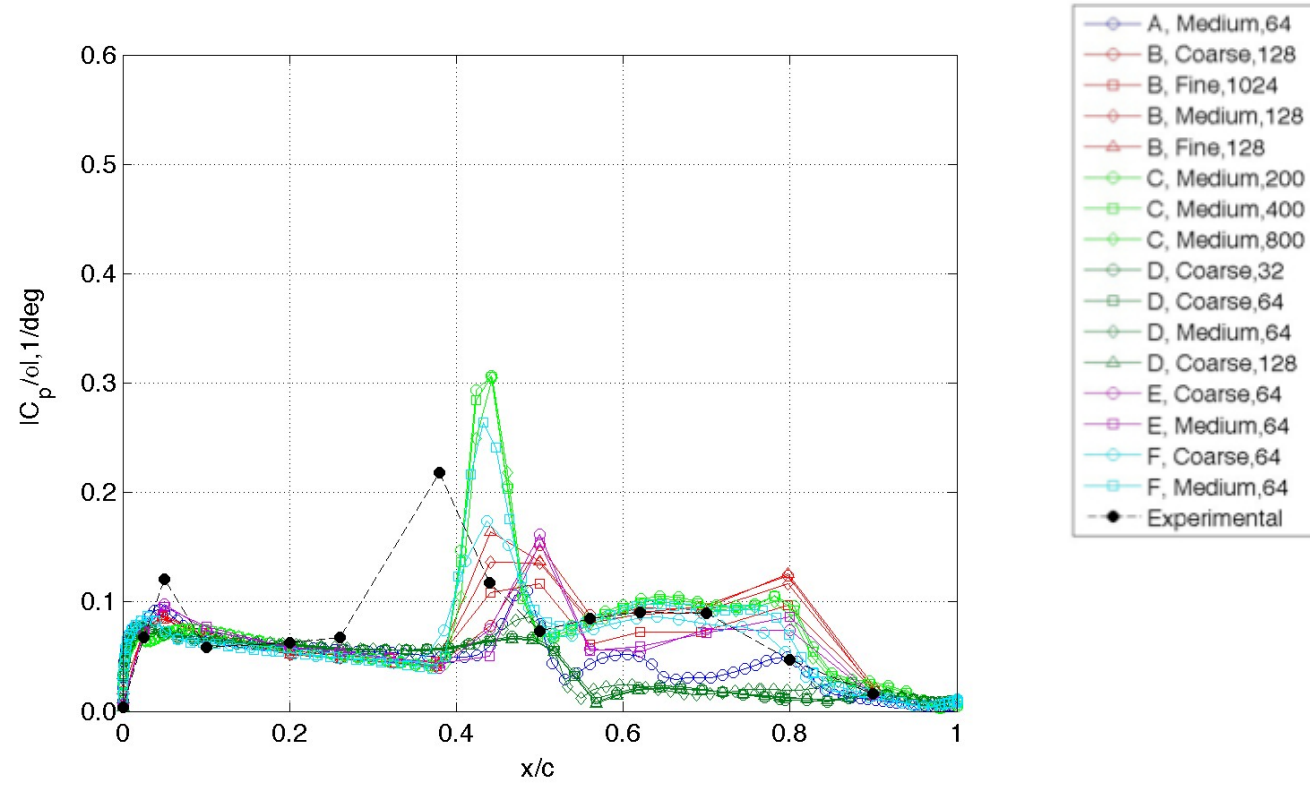

(a) Magnitude.

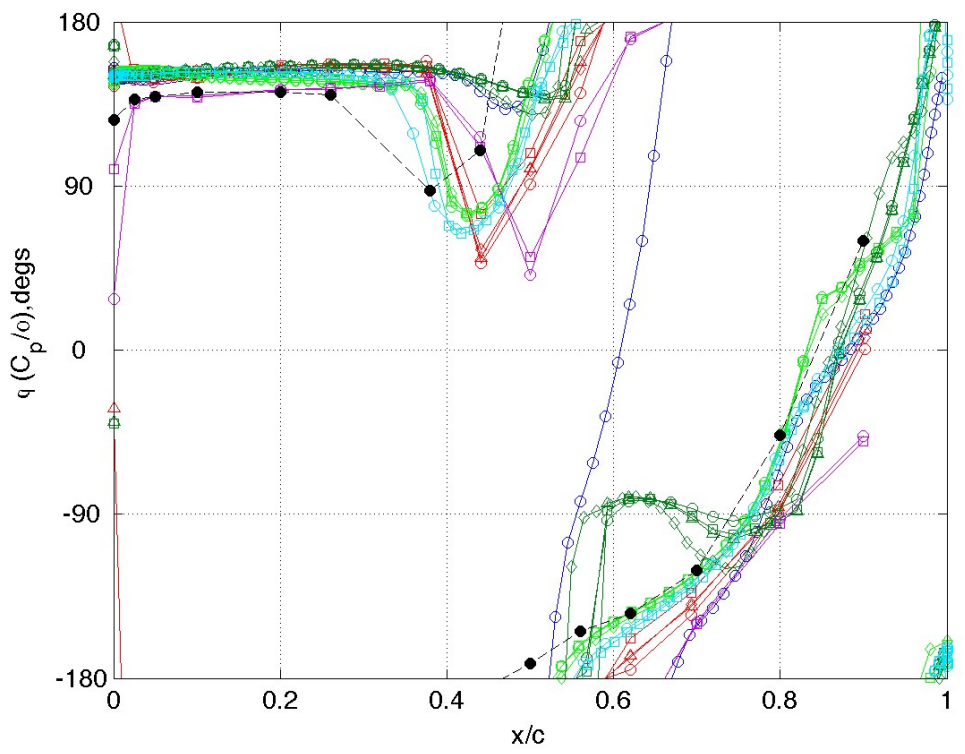

(b) Phase, deg.

Figure 46. Forced, $\alpha=2 \mathrm{deg}$, frequency $=20 \mathrm{~Hz}, \eta=0.588$. 

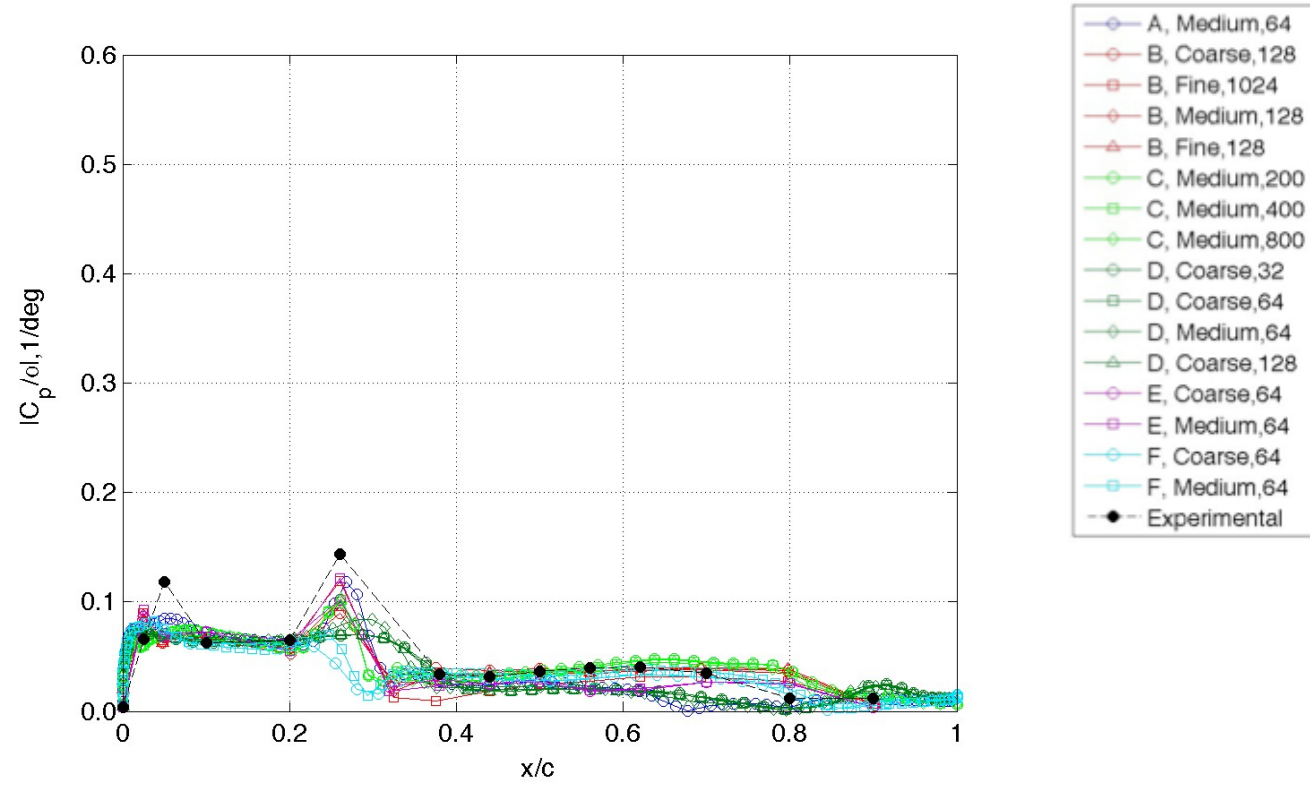

(a) Magnitude.

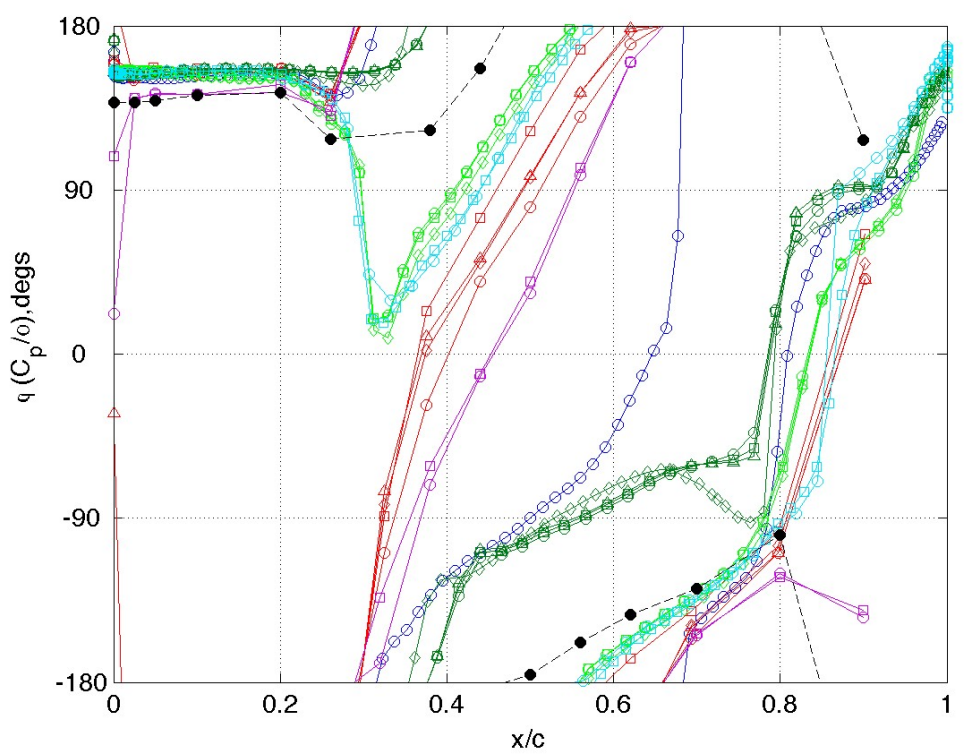

(b) Phase, deg.

Figure 47. Forced, $\alpha=2 \mathrm{deg}$, frequency $=20 \mathrm{~Hz}, \eta=0.809$. 

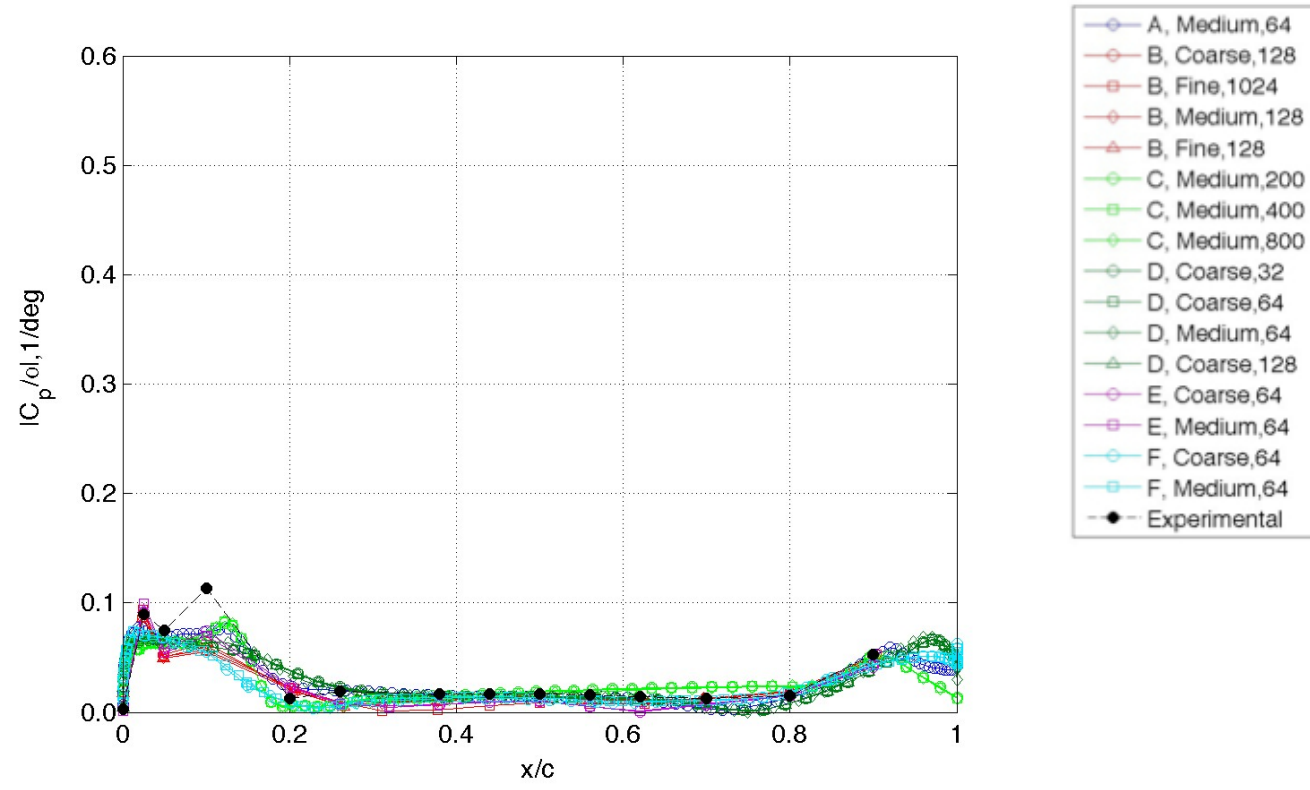

(a) Magnitude.

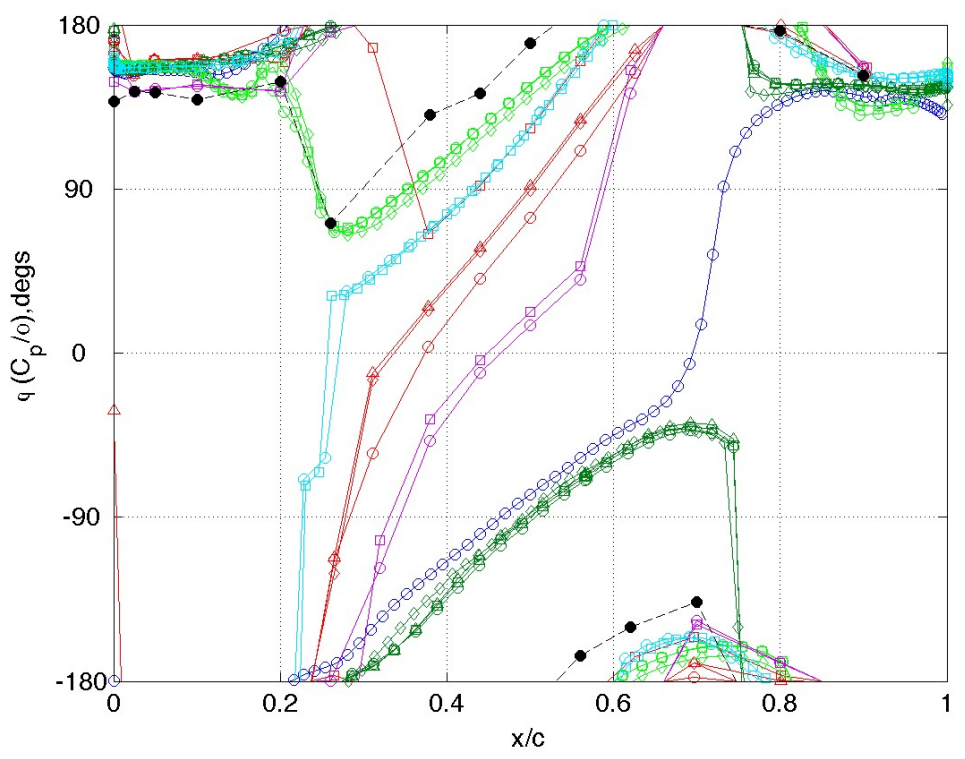

(b) Phase, deg.

Figure 48. Forced, $\alpha=2 \mathrm{deg}$, frequency $=20 \mathrm{~Hz}, \eta=0.951$. 

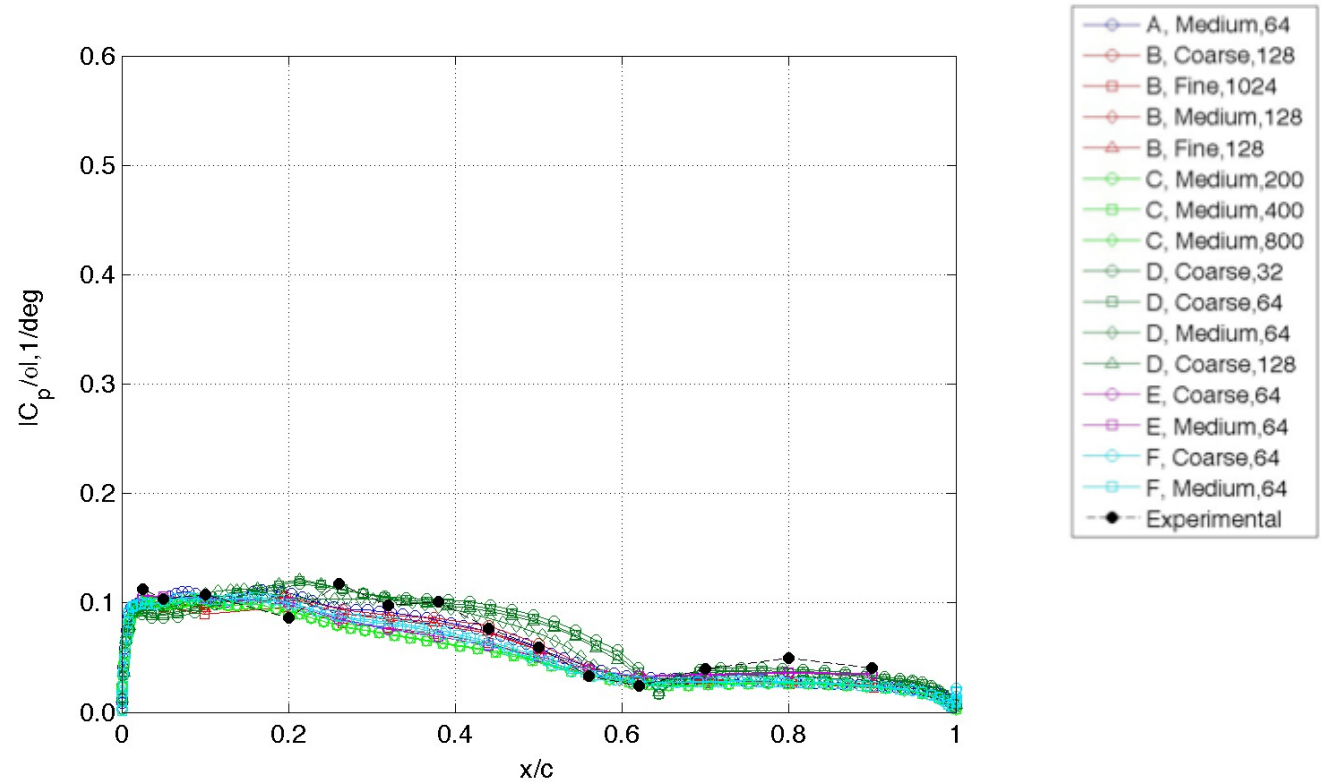

(a) Magnitude.

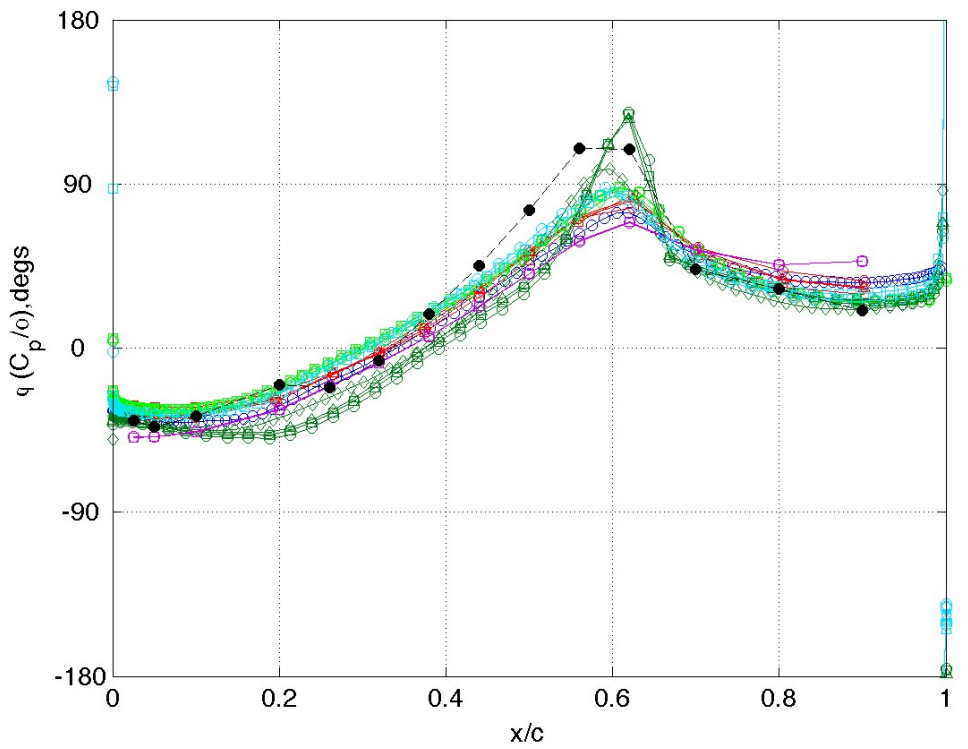

(b) Phase, deg.

Figure 49. Forced, $\alpha=2 \mathrm{deg}$, frequency $=20 \mathrm{~Hz}, \eta=0.309$. 

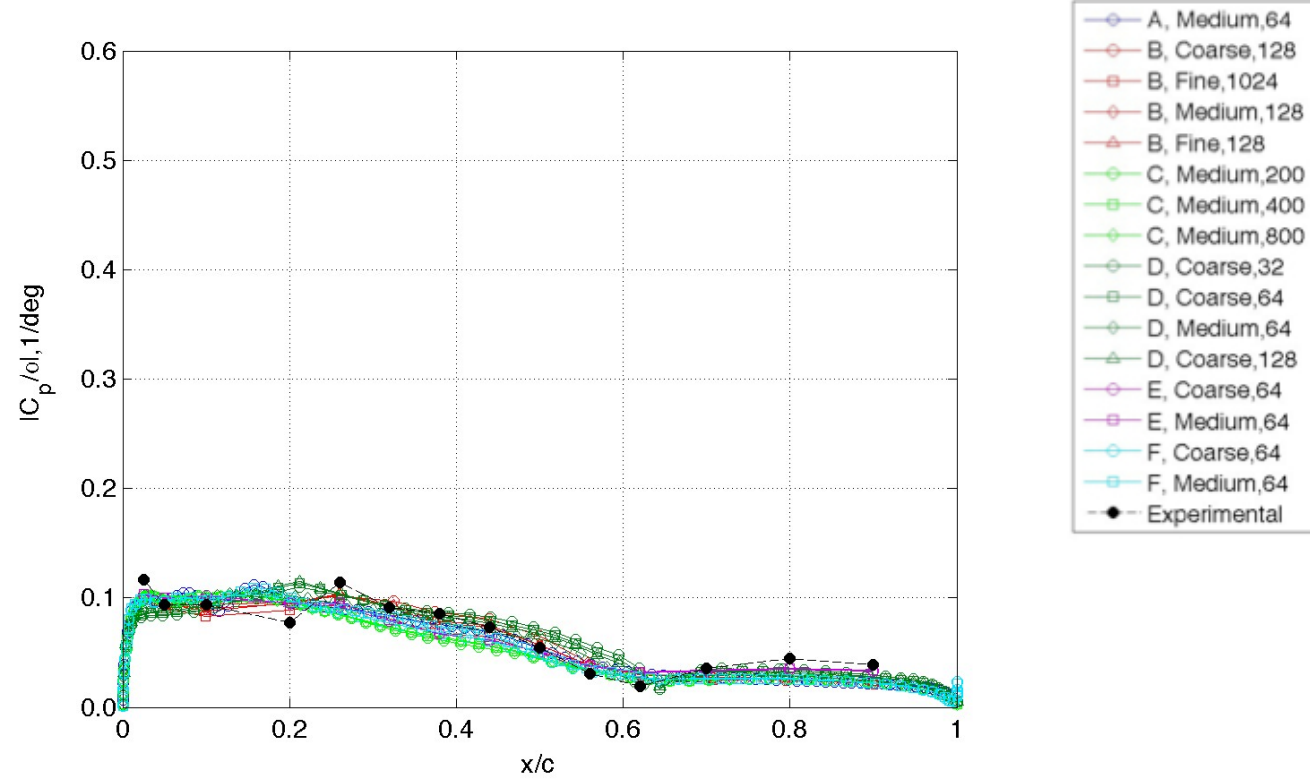

(a) Magnitude.

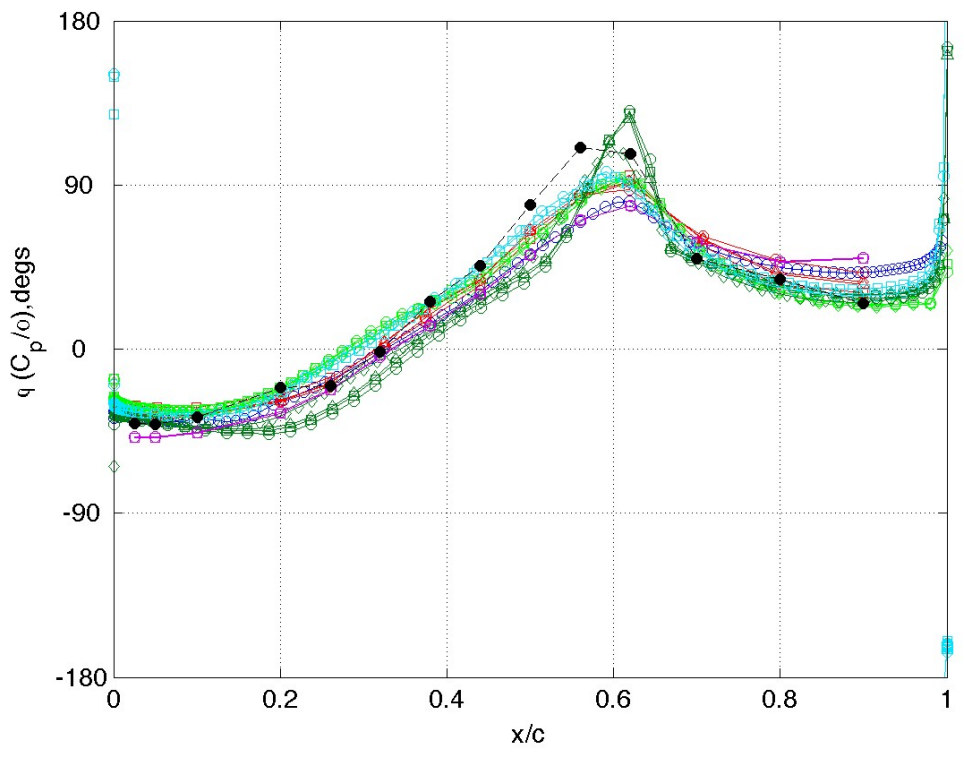

(b) Phase, deg.

Figure 50. Forced, $\alpha=2 \mathrm{deg}$, frequency $=20 \mathrm{~Hz}, \eta=0.588$. 

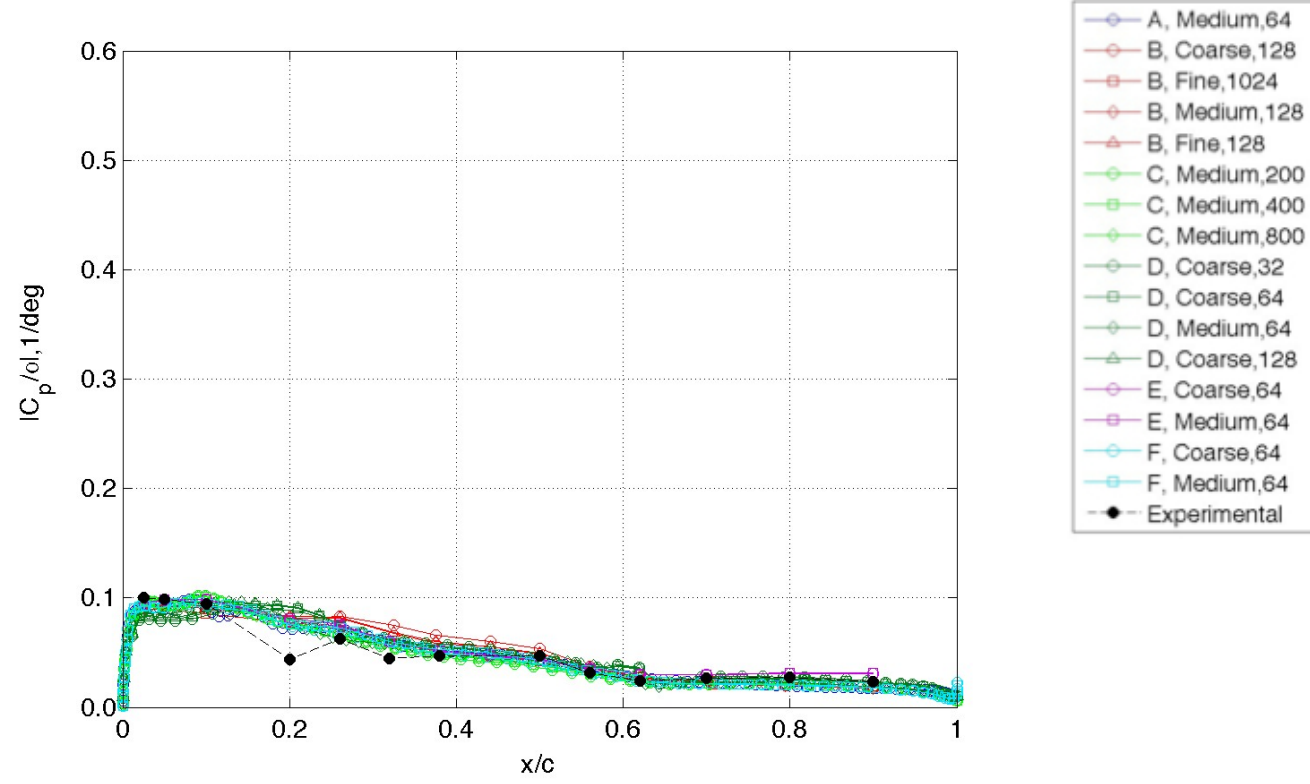

(a) Magnitude.

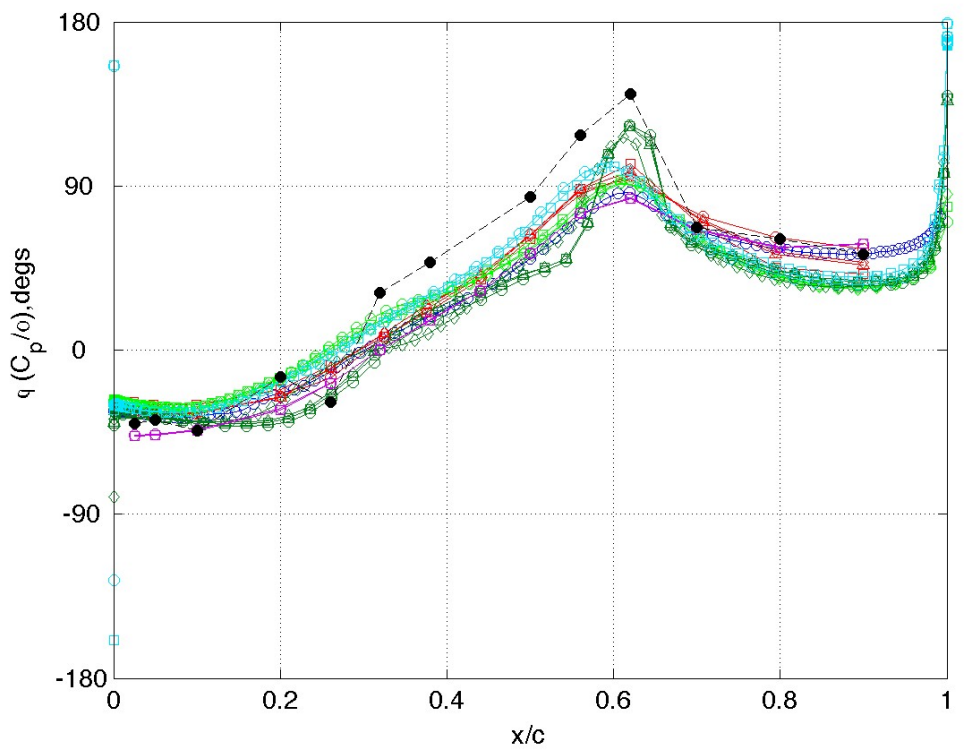

(b) Phase, deg.

Figure 51. Forced, $\alpha=2$ deg, frequency $=20 \mathrm{~Hz}, \eta=0.809$. 

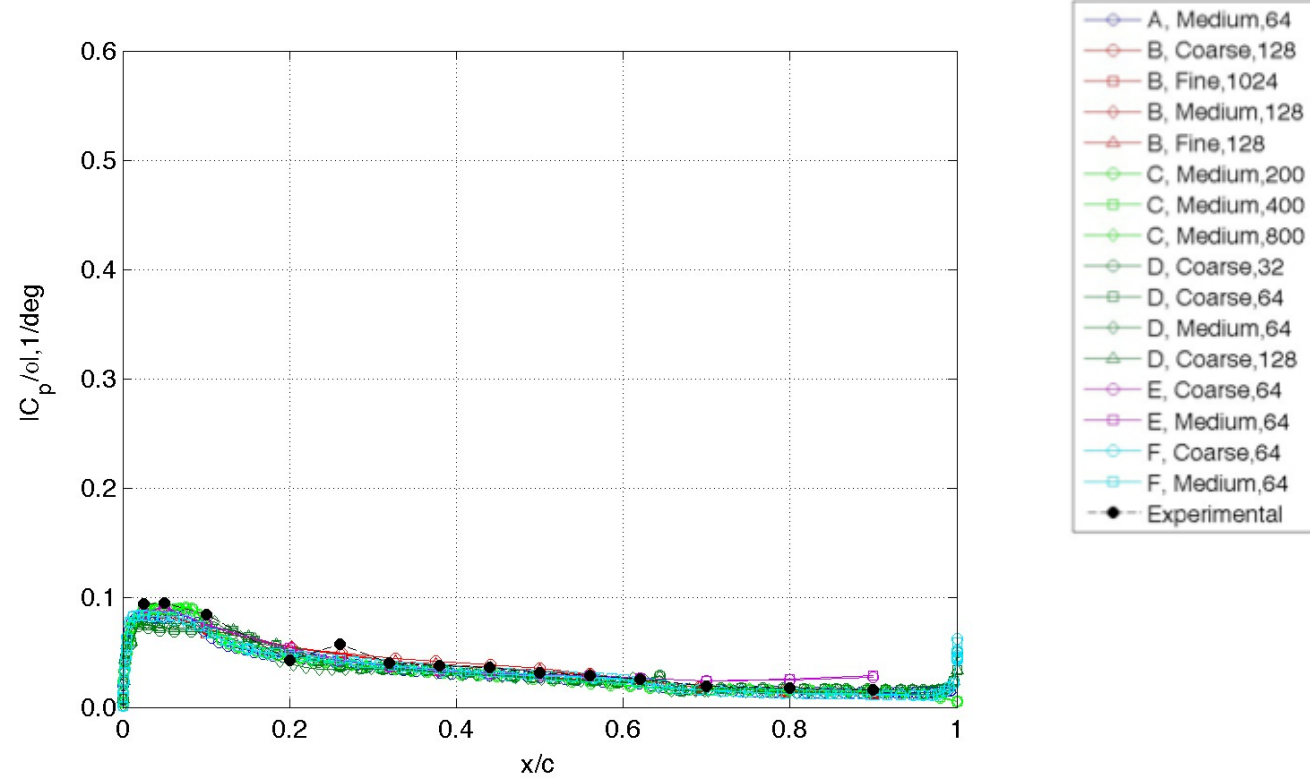

(a) Magnitude.

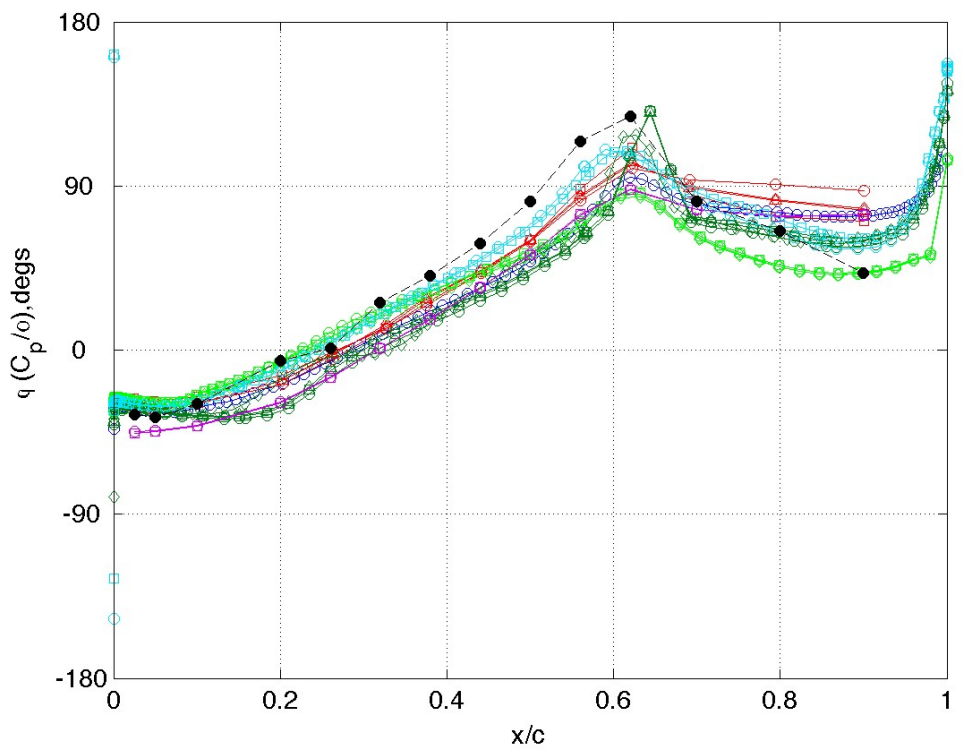

(b) Phase, deg.

Figure 52. Forced, $\alpha=2 \mathrm{deg}$, frequency $=20 \mathrm{~Hz}, \eta=0.951$. 


\section{Appendix E}

This appendix compares Analyst E results which include mean pressure distributions of forced oscillations, unforced steady-state computations and experimental unforced data
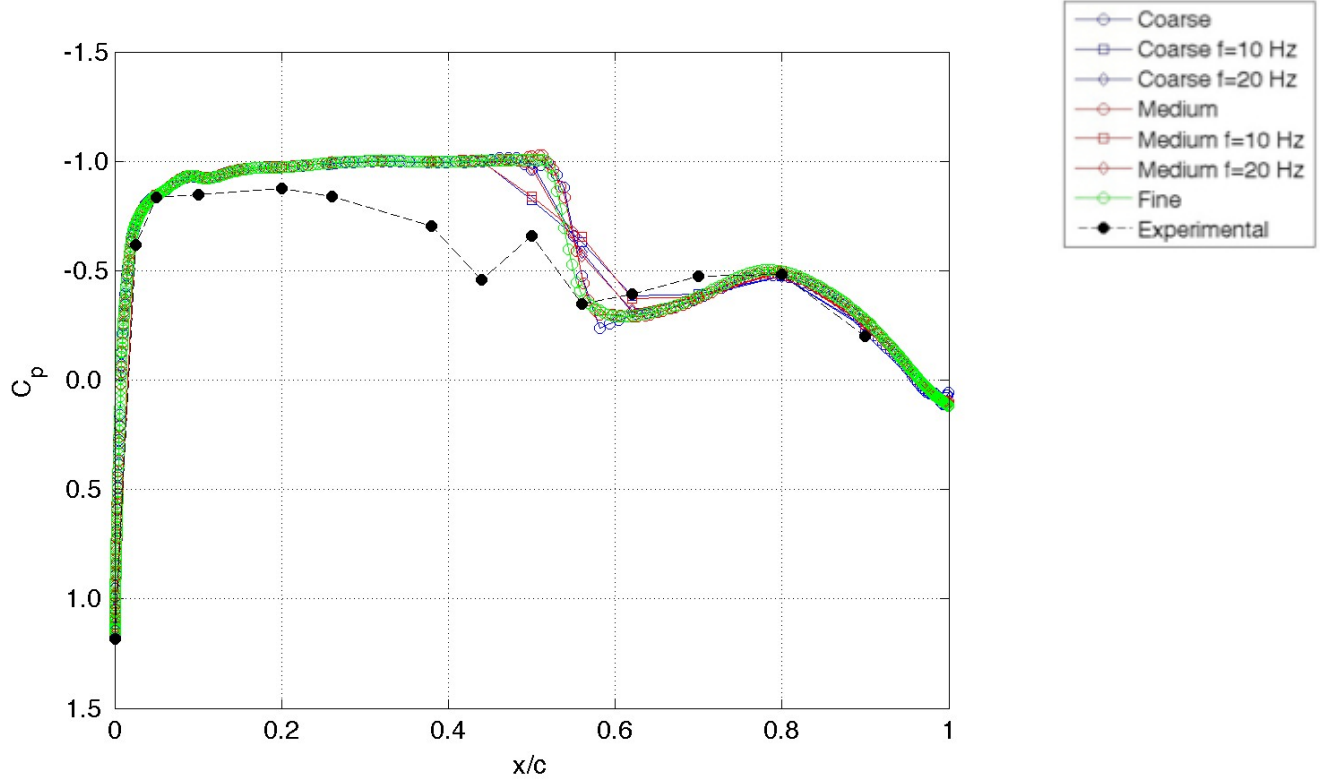

(a) Upper surface.

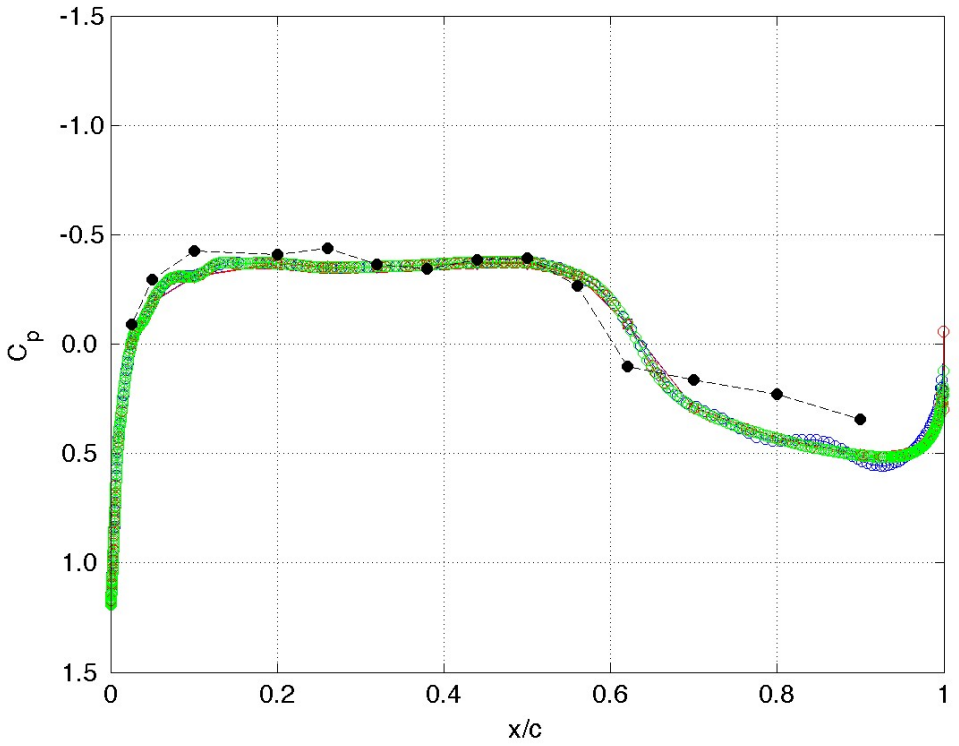

(b) Lower surface.

Figure 53. Unforced, $\alpha=2$ deg, $\eta=0.309$, analyst $E$ results only. 


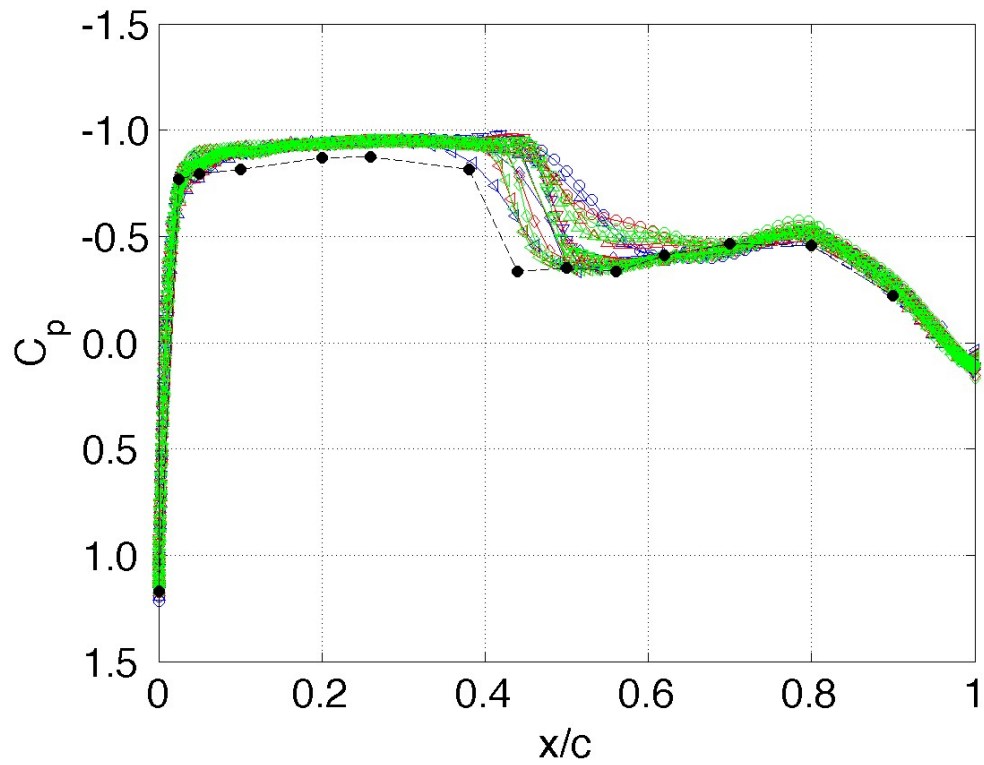

- Coarse
- Coarse $\mathrm{f}=10 \mathrm{~Hz}$
- Coarse $\mathrm{f}=20 \mathrm{~Hz}$
- Medium
- Medium $\mathrm{f}=10 \mathrm{~Hz}$
- Medium $\mathrm{f}=20 \mathrm{~Hz}$
- Fine
- Experimental

(a) Upper surface.

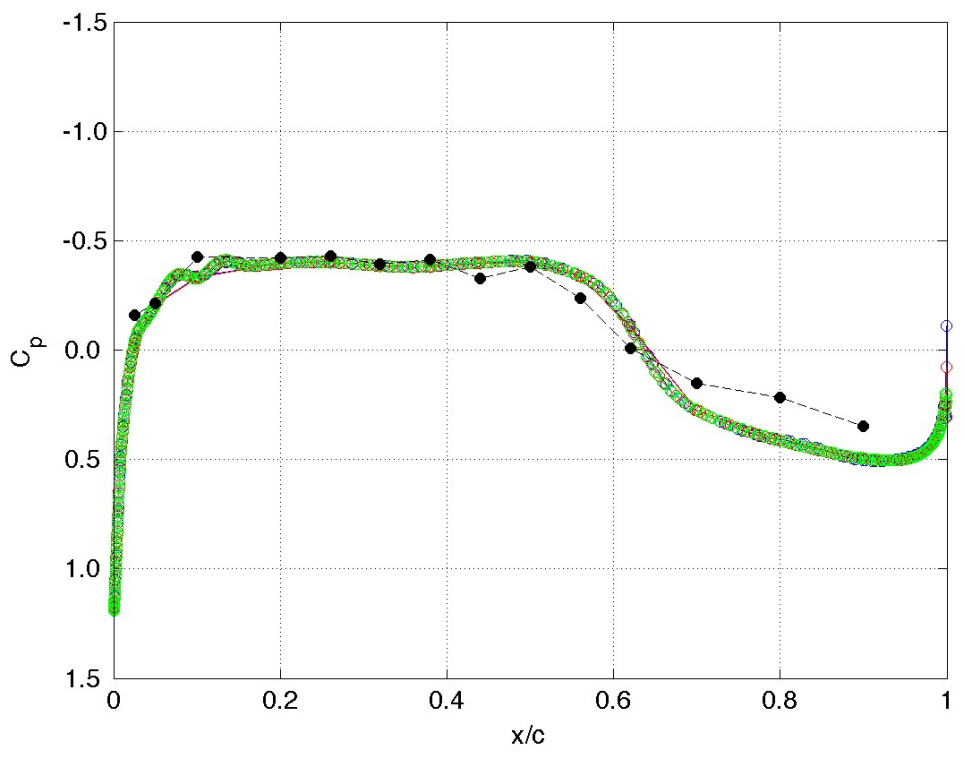

(b) Lower surface.

Figure 54. Unforced, $\alpha=2 \mathrm{deg}, \eta=\mathbf{0 . 5 8 8}$, analyst $\mathbf{E}$ results only. 

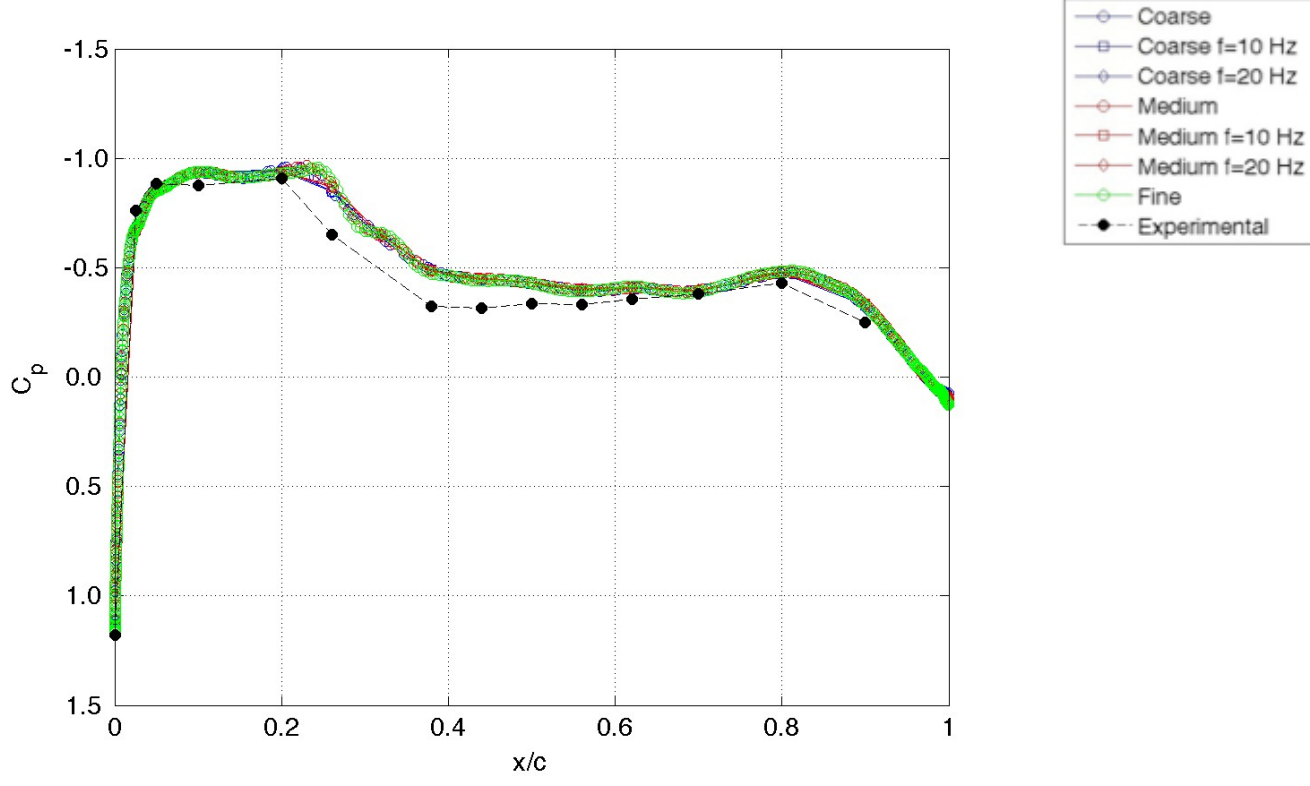

(a) Upper surface.

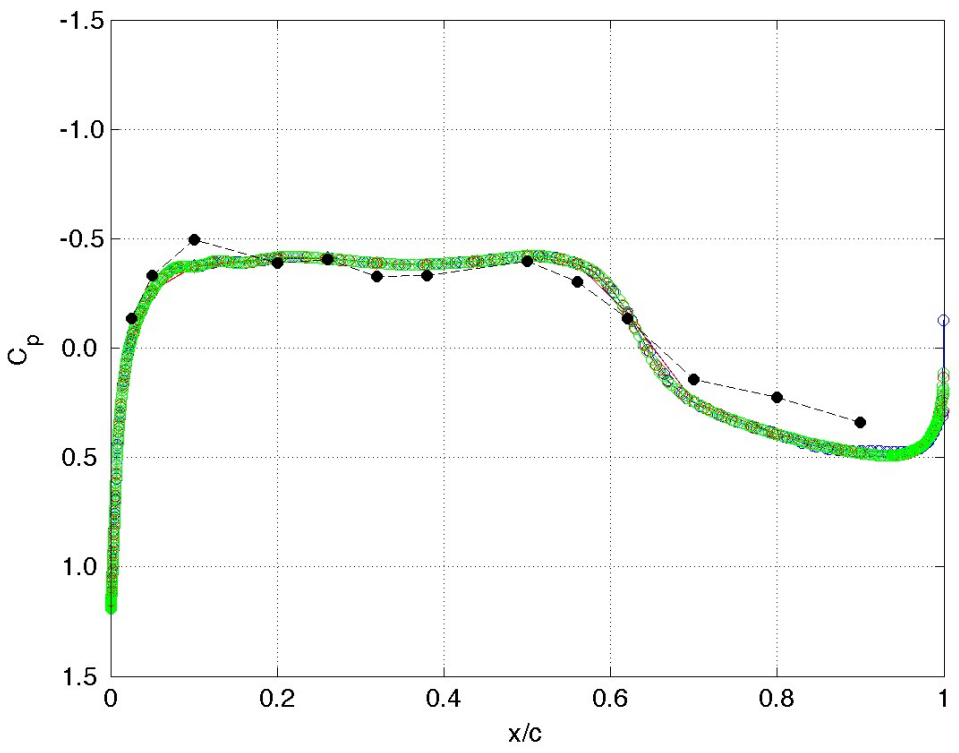

(b) Lower surface.

Figure 55. Unforced, $\alpha=2$ deg, $\eta=0.809$, analyst $\mathbf{E}$ results only. 

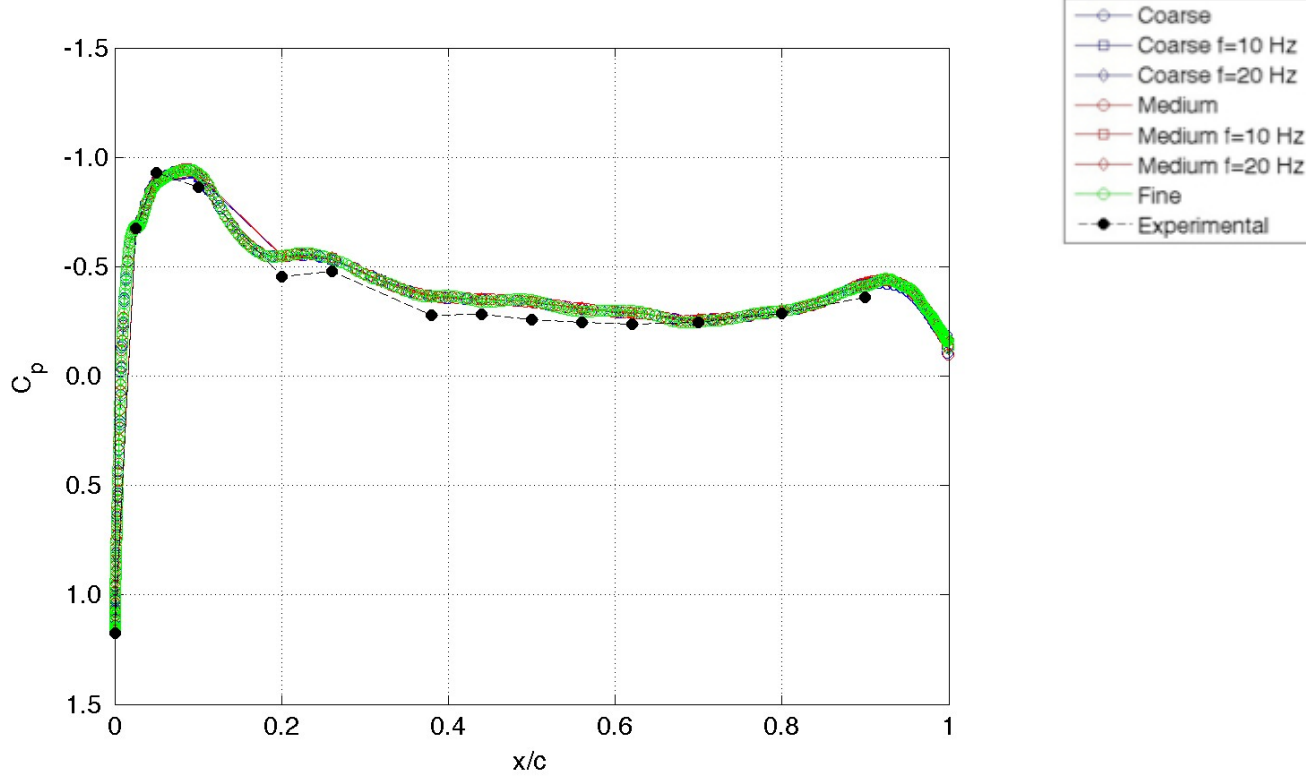

(a) Upper surface.

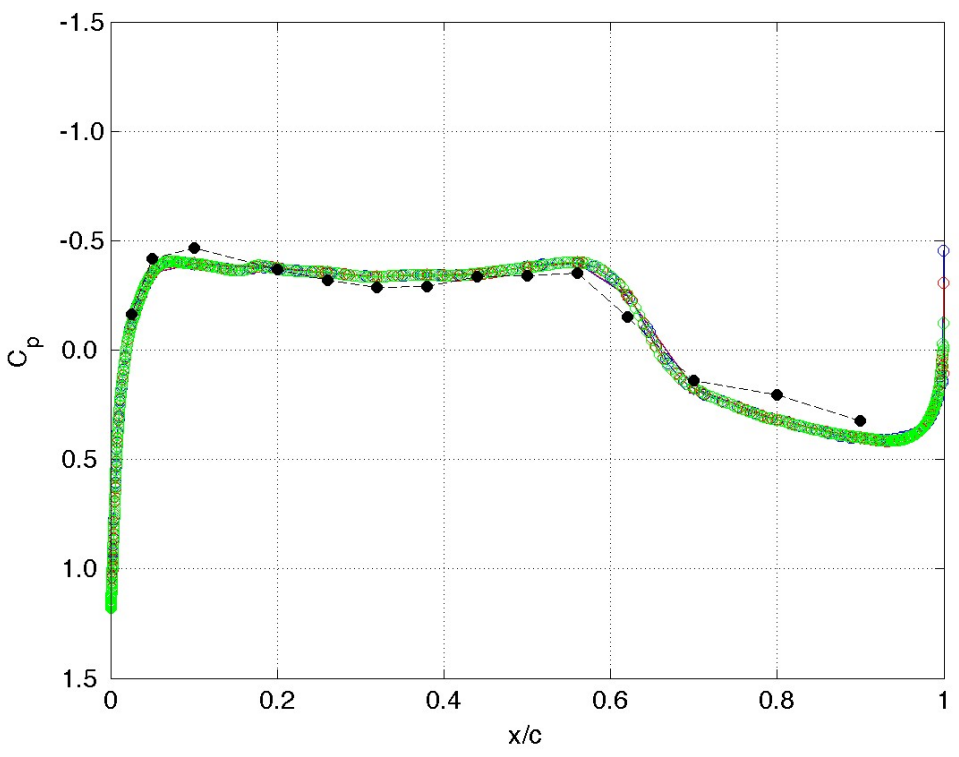

(b) Lower surface.

Figure 56. Unforced, $\alpha=2 \mathrm{deg}, \eta=\mathbf{0 . 9 5 1}$, analyst $\mathbf{E}$ results only. 